\title{
MITIGATION OF FLOODING DISRUPTION ATTACKS IN LINK STATE MOBILE AD HOC NETWORKS
}

by

Gimer Amilcar Cervera Evia

\author{
A Thesis Submitted to \\ the Faculty of Graduate and Post Doctoral Affairs \\ in Partial Fulfillment of \\ the Requirements for the Degree of \\ DOCTOR OF PHILOSOPHY \\ School of Computer Science \\ at \\ CARLETON UNIVERSITY
}

Ottawa, Ontario

May, 2012

(C) Copyright by Gimer Amilcar Cervera Evia, 2012 
Library and Archives

Canada

Published Heritage

Branch

395 Wellington Street

Ottawa ON K1A ON4

Canada
Bibliothèque et

Archives Canada

Direction du

Patrimoine de l'édition

395 , rue Wellington

Ottawa ON K1A ON4

Canada
Your file Votre référence

ISBN: 978-0-494-89325-8

Our file Notre référence

ISBN: $978-0-494-89325-8$

\section{NOTICE:}

The author has granted a nonexclusive license allowing Library and Archives Canada to reproduce, publish, archive, preserve, conserve, communicate to the public by telecommunication or on the Internet, loan, distrbute and sell theses worldwide, for commercial or noncommercial purposes, in microform, paper, electronic and/or any other formats.

The author retains copyright ownership and moral rights in this thesis. Neither the thesis nor substantial extracts from it may be printed or otherwise reproduced without the author's permission.
AVIS:

L'auteur a accordé une licence non exclusive permettant à la Bibliothèque et Archives Canada de reproduire, publier, archiver, sauvegarder, conserver, transmettre au public par télécommunication ou par l'Internet, prêter, distribuer et vendre des thèses partout dans le monde, à des fins commerciales ou autres, sur support microforme, papier, électronique et/ou autres formats.

L'auteur conserve la propriété du droit d'auteur et des droits moraux qui protege cette thèse. $\mathrm{Ni}$ la thèse ni des extraits substantiels de celle-ci ne doivent être imprimés ou autrement reproduits sans son autorisation.
In compliance with the Canadian Privacy Act some supporting forms may have been removed from this thesis.

While these forms may be included in the document page count, their removal does not represent any loss of content from the thesis.
Conformément à la loi canadienne sur la protection de la vie privée, quelques formulaires secondaires ont été enlevés de cette thèse.

Bien que ces formulaires aient inclus dans la pagination, il n'y aura aucun contenu manquant. 
Dedicated to my beloved parents, Gimer and Caricia 


\section{Abstract}

The Optimized Link State Routing (OLSR) is a proactive link state routing protocol, designed exclusively for Mobile Ad Hoc Networks (MANETs). OLSR is based on the selection of Multipoint Relays (MPRs) as a mechanism to reduce the size and number of control traffic messages flooded in the network. OLSR is not secure by design. A misbehaving node can affect the topology map acquisition process by interrupting the flooding of control information or disturbing the MPR selection process. This kind of attacks can be carried out in networks with cryptographic capabilities. OLSR is defined in RFC3626 and considers the selection of MPRs with additional coverage to increase the amount of reported information. Nevertheless, the overhead of the network increases due to the added number of control traffic messages. We propose a $k$-robust-MPR selection. Every node selects, when possible, $k+1$ disjoint MPR sets. The union of those sets, is a $k$-robust-MPR set. Our $k$-robust-MPR selection mitigates the effect of control traffic attacks and reduces the overhead generated by redundant control messages. Hierarchical OLSR (HOLSR) has been proposed to address scalability in MANETs. HOLSR implements the MPR flooding mechanism for intra-cluster and inter-cluster communications. We implement our $k$ robust-MPR selection to mitigate the effect of flooding disruption attacks at any hierarchical level. HOLSR implements Cluster ID Announcement (CID) messages to organize the network in clusters. We propose algorithm Hash-Chained_CID_Dissemination (HCCD) to detect and discard invalid CID messages. Multipath OLSR (MP-OLSR) is proposed to increase the resilience against failures in MANETs. Besides the effect of flooding disruption attacks, in MP-OLSR, nodes obtain only partial link-state information. MP-OLSR proposes control traffic messages with redundant information to construct disjoint paths. However, this increases considerably the size of the messages. We propose a Disjoint Multipath OLSR (DM-OLSR) strategy to construct, when possible, $t+1$ strictly disjoint paths. DM-OLSR relies on the $k$-Robust-MPR selection to enhance the network topology view and the route computation phases. 


\section{Acknowledgements}

I would like to express my sincere gratitude to my supervisors Dr. Evangelos Kranakis and Dr. Michel Barbeau for their guidance during the completion of my research. Also, I want to thank to Dr. Joaquin Garcia-Alfaro for his friendship and advice during all this process. Special thanks to Mathematics of Information Technology and Complex Systems (MITACS), National Council of Science and Technology (CONACYT), Ministry of Education of Mexico (SEP, Program for Academic Improvement) and the Universidad Tecnológica Metropolitana (UTM) for their support. Lastly but not least, to my family and friends for all their patience and support. 


\section{Table of Contents}

Abstract

Acknowledgements iv

List of Tables $\quad$ ix

List of Figures $\quad x$

$\begin{array}{lll}\text { Chapter } 1 & \text { Introduction } & 1\end{array}$

1.1 Introduction to Mobile Ad Hoc Networks . . . . . . . . . . . . 1

1.2 Characteristics of MANETs ................ 3

1.3 Routing Protocols for MANETs ................ 4

1.3.1 Reactive Routing Protocols . . . . . . . . . . . . . . 5

1.3.2 Proactive Routing Protocols .............. 8

1.3.3 Hybrid Routing Protocols ................. 10

1.4 Security issues in MANETs . . . . . . . . . . . . . . 11

1.4.1 Passive Attacks ................... 11

1.4 .2 Active Attacks ..................... 12

1.5 Summary of Contributions . . . . . . . . . . . . 13

1.5.1 Structure of the thesis ............... 15

1.5.2 Publications ................... 16

Chapter 2 Optimized Link State Routing Protocol 17

2.1 Introduction . . . . . . . . . . . . . . . 17

2.2 Neighbor Discovery Process . . . . . . . . . . . . . 18

2.3 MPR Computation . . . . . . . . . . . . . . . . . . . . 19

2.4 Network Topology Discovery . . . . . . . . . . . . . . 20

2.5 Format of Packets and Messages in OLSR . . . . . . . . . . . . 22

2.5.1 Packet and Message Header ... . . . . . . . . . . 22 
2.5.2 Hello Message Format . . . . . . . . . . . . . . . 24

2.5.3 TC Message Format . . . . . . . . . . . . . . 26

2.5.4 MID and HNA Message Format $\ldots \ldots \ldots \ldots \ldots$

2.6 Routing Table Calculation $\ldots \ldots \ldots \ldots \ldots \ldots \ldots$

2.7 Example of an OLSR-based network . . . . . . . . . . . . 29

2.8 OLSR Security Considerations $\ldots \ldots \ldots \ldots \ldots \ldots$

$\begin{array}{lll}\text { Chapter } 3 & \text { Security Issues in OLSR } & 31\end{array}$

3.1 Security Issues in $\operatorname{OLSR} \ldots \ldots \ldots \ldots \ldots \ldots$

3.2 Flooding Disruption Attacks $\ldots \ldots \ldots \ldots \ldots$

3.2.1 Incorrect MPR Selection . . . . . . . . . . . . . . . . . 34

3.2 .2 Incorrect Relaying $\ldots \ldots \ldots \ldots \ldots \ldots$

Chapter $4 \quad$ Survey of the Literature 39

4.1 Introduction . . . . . . . . . . . . . . . . . . . . 39

4.1 .1 Cryptographic Mechanisms . . . . . . . . . . 40

4.1.2 Intrusion Detection Systems . . . . . . . . . . . . . 42

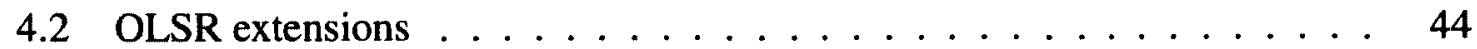

$4.2 .1 \quad$ Scalability $\ldots \ldots \ldots \ldots \ldots \ldots \ldots \ldots \ldots$

4.2 .2 Multicasting $\ldots \ldots \ldots \ldots \ldots \ldots \ldots \ldots$

4.2 .3 Multipath Routing $\ldots \ldots \ldots \ldots \ldots$

Chapter 5 Mitigation of Flooding Disruption Attacks in OLSR 47

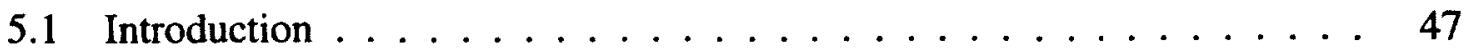

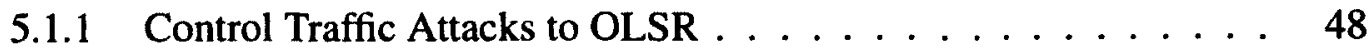

5.1 .2 Contributions of the Chapter . . . . . . . . . . . . . . 49

5.1 .3 Related Work . . . . . . . . . . . . . . . . . . . 49

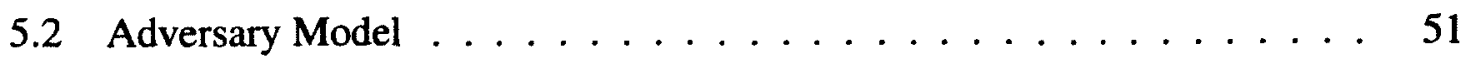

5.3 MPR Computation with Additional Coverage $\ldots \ldots \ldots \ldots \ldots . \ldots 52$

5.3.1 RFC3626's MPR Coverage Parameter . . . . . . . . . . . . 53

$5.3 .2 k$-Robust-MPR Selection . . . . . . . . . . . . . . 57 
5.3.3 Correctness of the Functions . . . . . . . . . . . . 57

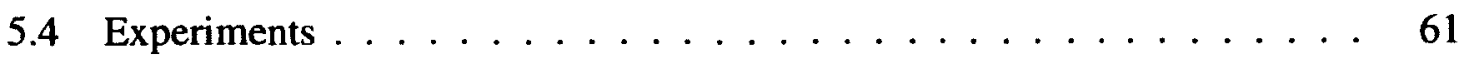

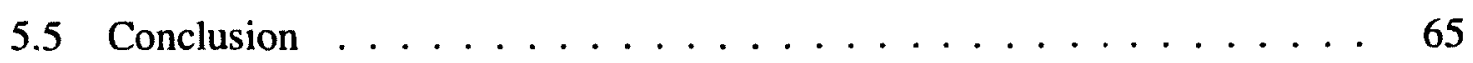

Chapter 6 Security Issues in Hierarchical OLSR 66

6.1 Introduction ......................... 66

6.2 Hierarchical OLSR (HOLSR) . . . . . . . . . . . . . . 68

6.2 .1 Cluster Formation . . . . . . . . . . . . . 69

6.2 .2 Cluster Head Message Exchange . . . . . . . . . . . 71

6.2.3 Topology Control Propagation ... . . . . . . . . . 72

6.3 Other Hierarchical Approaches . . . . . . . . . . . . . 73

6.3 .1 Cluster OLSR . . . . . . . . . . . . . 73

6.3.2 The Multi-level OLSR Routing Using the HNA Extension . . . 75

6.3 .3 Tree Clustering . . . . . . . . . . . . . 76

6.4 Flooding Disruption Attacks in HOLSR . . . . . . . . . . . . 79

6.5 Countermeasures ........................ 79

6.5.1 MPRs with Additional Coverage . . . . . . . . . . . . 80

6.5.2 Redundant Information . . . . . . . . . . . . . 81

6.5.3 Non-Source Dependent Mechanism . . . . . . . . . . . 81

6.5.4 Future Work: Locally Proactive HOLSR . . . . . . . . . . . 82

6.5 .5 Experiments ..................... 83

6.6 Cluster Formation Attack against the HOLSR protocol . . . . . . . . 86

6.6 .1 Adversary Model . . . . . . . . . . . . . . 87

6.6.2 Handling the Attack with the use of Hash Chains . . . . . . . 88

6.6.3 Wormhole Attack in HOLSR ............... 91

6.7 Results and Simulations Setup . . . . . . . . . . . . . . . 94

6.8 Related Work . . . . . . . . . . . . . . . 96

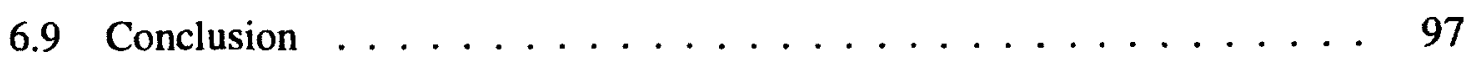

Chapter 7 Security Issues in Multipath OLSR 99

7.1 Introduction . . . . . . . . . . . . . . . 99 
7.1 .1 Contributions . . . . . . . . . . . . . . 101

7.2 Related Work . . . . . . . . . . . . . . . 102

7.3 Multipath OLSR . . . . . . . . . . . . . . . . . . . . . 104

7.3.1 Route Computation . . . . . . . . . . . . . . . . . 106

7.4 MP-OLSR Drawbacks and Security Vulnerabilities . . . . . . . . 108

7.4.1 Partial Network Topology View . . . . . . . . . . 110

7.4.2 Flooding Disruption Attacks in Multipath OLSR . . . . . . 111

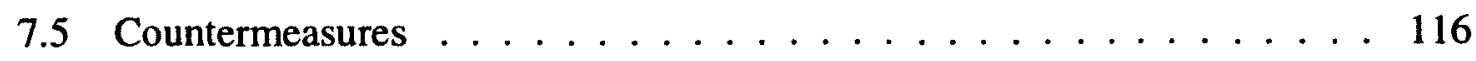

7.5.1 MPR Selection with additional Coverage $\ldots \ldots \ldots \ldots 116$

7.5.2 Disjoint Multipath OLSR . . . . . . . . . . . . . . 117

7.5.3 Practical Example . . . . . . . . . . . . . . . 120

7.5 .4 Correctness of the Functions . . . . . . . . . . . . . 121

7.6 Simulations and Results . . . . . . . . . . . . . . . . . . . 122

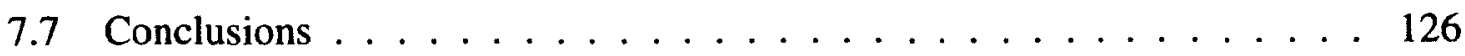

$\begin{array}{lll}\text { Chapter } 8 & \text { Conclusions and Future Work } & 127\end{array}$

8.1 Conclusions . . . . . . . . . . . . . . . . . . . . . . 127

8.1.1 Summary of Contributions and Results $\ldots \ldots \ldots \ldots$

8.2 Future Work . . . . . . . . . . . . . . . . . . . 130

8.2 .1 Phantom node attack in OLSR . . . . . . . . . . 130

8.2.2 Locally proactive OLSR-based networks . . . . . . . . 130

8.2.3 MPR Selection with Additional Coverage in Dynamic Networks . 131

8.2.4 Colluding Flooding Disruption Attacks in OLSR . . . . . . 131

8.2.5 Handling other cluster formation attacks in HOLSR . . . . . . 131

$\begin{array}{ll}\text { Bibliography } & 134\end{array}$ 


\section{List of Tables}

Table 2.1 Summary of control traffic messages in OLSR. MID and HNA messages are optional. . . . . . . . . . . . 21

Table 5.1 MPR computation examples in figure $5.3 \ldots \ldots \ldots \ldots \ldots$

Table 5.2 NS-2 and scenario parameters. . . . . . . . . . . . . . 64

Table 6.1 Summary of control traffic messages in HOLSR. . . . . . . 72

Table 6.2 Comparison of OLSR-based hierarchical approaches. All approaches implement Hello and TC message for intra-cluster communications. $\quad 78$

Table 7.1 MPR selectors with or without additional coverage ( $k$ is equal to two). 111 


\section{List of Figures}

Figure 1.1 Examples of a cellular and an ad hoc network. . . . . . . . 2

Figure 1.2 Examples of radiation patters of antennas. . . . . . . . . . 3

Figure 1.3 Examples of the hidden station problem in MANETs. . . . . . . 3

Figure 2.1 MPR based mechanism against the classical flooding. Consider gray nodes as the originators of a TC message and black nodes as MPRs......................... 18

Figure 2.2 Example of an OLSR packet. . . . . . . . . . . . 23

Figure 2.3 Example of a Hello message. . . . . . . . . . . . . . 25

Figure 2.4 Example of a TC message. . . . . . . . . . . . . . . 26

Figure 2.5 Optional OLSR messages. . . . . . . . . . . . . . . 27

Figure 2.6 Example of an OLSR-based network. . . . . . . . . . . . . . . . 29

Figure 3.1 Example of an OLSR-based network with misbehaving nodes $M_{1}$

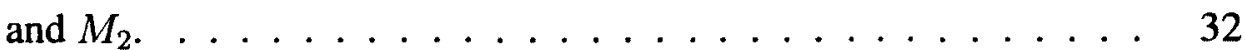

Figure 3.2 Taxonomy of flooding disruption attacks [24]. . . . . . . . . . 33

Figure 3.3 Flooding disruption due to identity spoofing attacks. In Fig. 3.3(a) node $x$ spoofs $d$ and reports an incorrect link between nodes $c$ and $d$ (one-hop address duplication). In Fig. 3.3(b), node $x$ spoofs $c$ and affects node $a$ 's MPR selection (two-hop address duplication). . . 35

Figure 3.4 Flooding disruption due to link spoofing attacks. In Fig. 3.4(a), node $x$ spoofs links to nodes $e$ and $c$. In Fig. 3.4(b), node $x$ spoofs links to nodes $e$ and the inexistent node $w . \ldots 36$

Figure 3.5 Flooding disruption due to protocol disobedience. In Fig. 3.5(a), node $x$ never selects a valid MPR set. In Fig. 3.5(b), node $x$ modifies and forwards incorrectly TC messages. . . . . . . . . . 37 
Figure 5.1 Example of a selfish and a slanderer attack. In (a), node 5 is a selfish node and has been selected by node 2 as an MPR. In (b), node 3 is a slanderer node and has been selected by node 1 as an MPR.

Figure 5.2 MPR selection in an OLSR network with and without additional coverage. Consider gray nodes as MPRs. . . . . . . . . . 53

Figure 5.3 Consider nodes 2, 3, 6 and 9 as MPRs, selected with no extra cov-

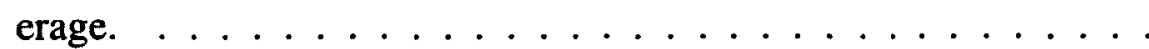

Figure 5.4 Comparative of functions $k$-covered-MPR and $k$-robust-MPR under the presence of selfish nodes. . . . . . . . . . . . 62

Figure 5.5 Comparative of functions $k$-covered-MPR and $k$-robust-MPR under the presence of slanderer nodes. . . . . . . . . . . .

Figure 6.1 Example of a hierarchical architecture with heterogeneous nodes.

Figure 6.2 Example of a Cluster OLSR network. Consider gray clusters as C-MPRs. . . . . . . . . . . . . . . 74

Figure 6.3 Example of a MORHE network. Consider black nodes as backbone nodes. ........................ 75

Figure 6.4 Tree clustering. Black nodes represents the roots of the tree. Branches of the trees are shown with solid lines between nodes. Links that are not branches are dashed. . . . . . . . . . . . 77

Figure 6.5 Flooding disruption due to link spoofing attack (phantom node). $\quad 83$

Figure 6.6 Comparison of functions NSD-MPR, $k$-covered-MPR, and $k$-robustMPR under the presence of selfish nodes. . . . . . . . . . . . 84

Figure 6.7 CID messages propagation. . . . . . . . . . . . . . 85

Figure 6.8 CID message attack. . . . . . . . . . . . . . . . . 88

Figure 6.9 Standard deviation of the average number of nodes per cluster testing different HOLSR networks with up to five malicious nodes and applying algorithm HCCD . . . . . . . . . . . . . 92

Figure 6.10 Wormhole attack in HOLSR. . . . . . . . . . . . . . . 93 
Figure 7.1 Example of constructing multiple paths in MP-OLSR. . . . . . . 107

Figure 7.2 Node $s$ perspective of the network topology with parameter TCR equal to two. Gray nodes represent MPRs. . . . . . . . . . . 110

Figure 7.3 Node $M_{1}$ is a misbehaving node. . . . . . . . . . . 111

Figure 7.4 Node $s$ perspective of the network topology after an identity spoofing attack. Node $M_{1}$ spoofs the identity of node $s \ldots \ldots 112$

Figure 7.5 Node $s$ perspective of the network topology after a link spoofing attack. Node $M_{1}$ spoofs links to nodes $s, a, g$ and $j \ldots \ldots 113$

Figure 7.6 Topology view of the network from the perspective of node $s$ and additional MPR coverage. . . . . . . . . . . . . 115

Figure 7.7 Network topology with edge cost from the perspective of $s$. . . . . 121

Figure 7.8 Simulations to compare MP-OLSR against our function DM-OLSR (95\% confidence interval). Number of TC messages and their average size. . . . . . . . . . . . . . . . . . . . 124

Figure 7.9 Simulations to compare MP-OLSR against our function DM-OLSR (95\% confidence interval). Number of constructed paths and performance ratio. ................... 125 


\section{Chapter 1}

\section{Introduction}

\subsection{Introduction to Mobile Ad Hoc Networks}

In this chapter, we describe some aspects of ad hoc networking, an overview of security issues and the contributions presented in this thesis. In wireless networks $[13,83]$, computers communicate with each other through a non-physical medium, using radio waves. A wireless network can be configured to function in either Basic Service Set (BSS) or Independent Basic Service Set (IBSS) mode [83]. In BSS mode or infrastructure mode, mobile devices communicate with each other through a non-mobile Base Station (BS) as access point. IBSS or ad hoc mode, allows wireless devices point-to-point communication, i.e., no access point nor fixed infrastructure is needed.

An ad hoc network consists of wireless devices that may be mobile and can communicate without the need of a fixed infrastructure. In a Mobile Ad Hoc Network (MANET), the network topology may change dynamically since nodes can move in an unpredictable manner $[19,13]$. Nodes are free to move at any speed in any direction and join or leave the network at any time. Nodes with heterogeneous capabilities (e.g., transmission range) can coexist. In [13], Agrawal and Zeng formally define a MANET as an autonomous system of nodes or Mobile Stations ( $M S s$ ) connected by a wireless medium. This network may be modeled in the form of an arbitrary communication graph.

In a MANET, every node is able to communicate directly with other nodes within its range. Nodes with direct communication are called neighbors. Any pair of nodes not directly connected, can communicate through a path formed by other nodes. MANETs are basically peer-to-peer, multihop wireless networks [13]. The established links can be either symmetric (i.e., links with the same characteristics in both directions) or asymmetric (i.e., links with different characteristics in each direction). Information packets are 


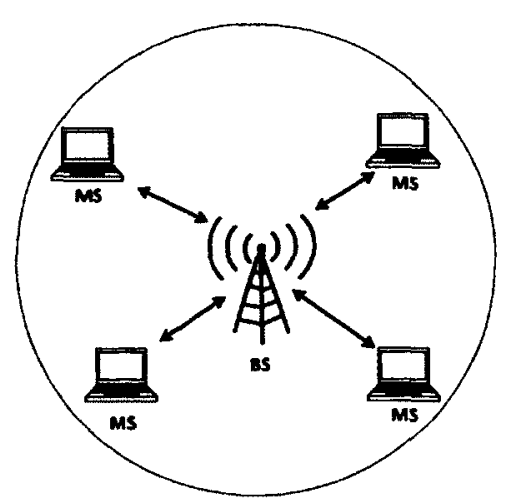

(a) A base station $(B S)$ and its network cell.

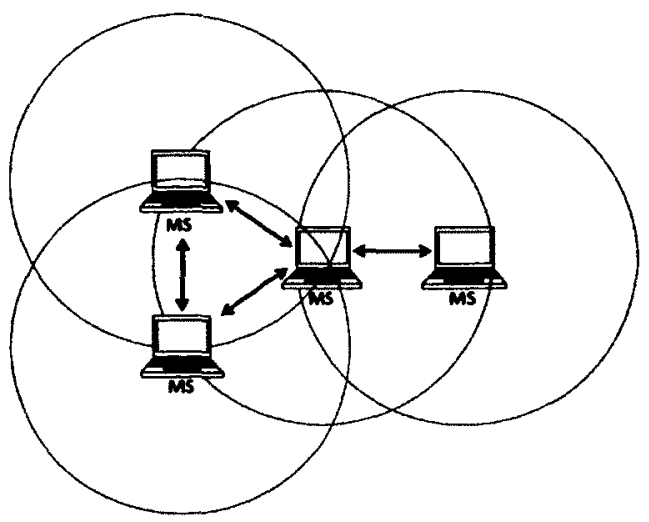

(b) Mobile stations $(M S)$ in ad hoc mode

Figure 1.1: Examples of a cellular and an ad hoc network.

transmitted in a store-and-forward manner by intermediate nodes, i.e., every node acts as a router. In a MANET, every node configures its network address and can resolve the way to reach any destination. There is no need for an address communication server, e.g., DHCP server. Fig. 1.1(a), presents an example of a wireless network. Every pair of MSs has to commnicate throug the BS.Fig. 1.1(b), shows an example of a network in ad hoc mode. $M S$ s within the same range can establish direct communication.

Ad hoc networks can operate in isolation or may have gateways to a fixed network. In this case, the ad hoc network operates as a stub network connected to a fixed network. A node with multiple interfaces can communicate with both the ad hoc and the external network (e.g., Internet). The node that acts as a gateway does not permit that external traffic transits through the stub network. Each node is equipped with a wireless transmitter and a receiver with an antenna which may be omnidirectional, i.e., the same power is transmitted in all directions, or directional, i.e., the power is transmitted in a specific direction. Fig. 1.2 shows an example of both types of antennas. In Fig. 1.2 (a), $M S_{1}$ and $M S_{2}$ receive the same amount of power from a omnidirectional antenna. In Fig. 1.2 (b), $M S_{2}$ receives more power than $M S_{1}$. MSs have limited radio range and a restricted view of the network. This causes the well-know hidden station problem $[83,13]$. Consider $M S_{1}$, $M S_{2}$ and $M S_{3}$ in Fig. 1.3. $M S_{1}$ and $M S_{3}$ are both in range of $M S_{2}$ but they are not in range of each other. The hidden station problems occurs if $M S_{3}$ is transmitting to $M S_{2}$, and $M S_{1}$ attempts to transmit at the same time. $M S_{1}$ is not aware that $M S_{2}$ is busy and 


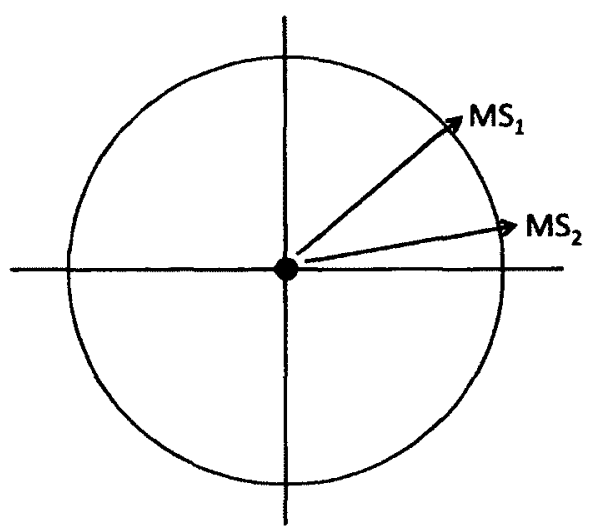

a) Omnidirectional

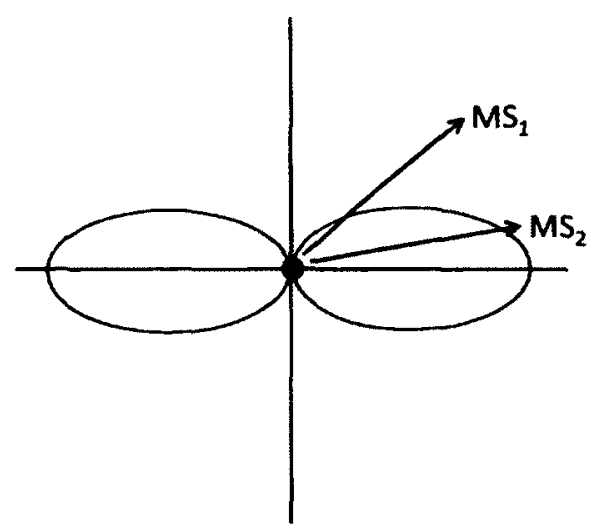

b) Directional

Figure 1.2: Examples of radiation patters of antennas.

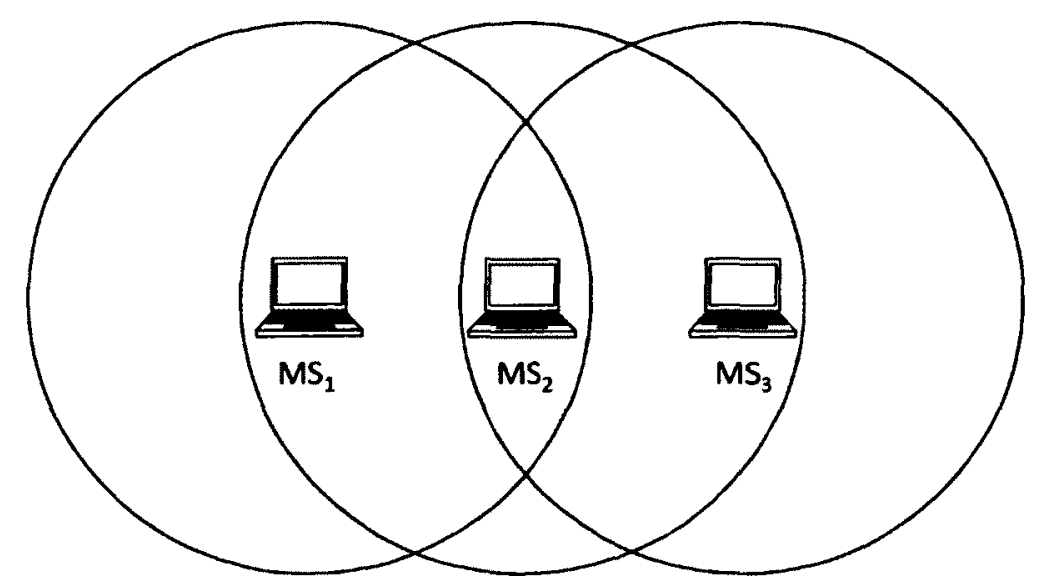

Figure 1.3: Examples of the hidden station problem in MANETs.

that a message collision will occur. $M S_{1}$ transmits when it should not. Also, $M S_{2}$ may mistakenly think that it should not attempt to transmit to avoid collisions. $M S_{2}$ abstains from transmitting, when it would not need to.

\subsection{Characteristics of MANETs}

The decentralized nature of MANETs provides additional robustness against the single point of failure in centralized approaches, e.g., BS or access point. MANETs can be deployed in scenarios where it is almost impossible to set up and maintain a wired network (e.g., a disaster area or a battlefield). MANETs have particular characteristics and restrictions [13]: 
1. Dynamic topologies: Nodes are free to move arbitrarily at different speeds. It is difficult to predict a node's movement and its trajectory. Therefore, the network topology may change randomly and unpredictably.

2. Limited bandwidth: Wireless links have lower capacity than wired networks. Nowadays however, some standards offer better data rates comparable to those of Ethernet (e.g., WiMAX). Additionally, the throughput in wireless communication is affected by the conditions of the environment, fading, noise or interference.

3. Energy-constrained: In a MANET, nodes may rely on limited batteries as source of energy. Nodes do not have enough transmitting power to reach all nodes in the network. This restricts the nodes communication to only the neighboring nodes.

4. Limitations of the medium: In a wireless network, is not possible to transmit and to listen at the same time. Message loss is more likely due to collisions and interference.

5. Limited physical security: In MANETs, data packets travel across the air. Hence, it is relatively easy for an intruder to eavesdrop data packets by operating in promiscuous mode and by using a packet sniffer. Nodes may also be affected by intentional jamming or denial of service (DoS) attacks.

\subsection{Routing Protocols for MANETs}

Routing is an important issue in MANETs. An efficient resources utilization is necessary due to the restricted capabilities of nodes. In a MANET, route computation must be distributed, as centralized routing in a dynamic network is not possible. Nodes only have a local knowledge of the network. According to Abolhasan et al. [7], an efficient and reliable routing strategy must consider the limited resources and being adaptable to the network conditions such as mobility, network size or traffic density. In wired networks there are two main routing approaches: link-state and distance vector [19, 13, 7]. In link-state routing, every node floods the network with the link-state information of its neighboring nodes. Receivers update their view of the network and utilize a shortest path algorithm to chose the next hop to each destination. In distance-vector routing, every node maintains a set of 
distances for every destination. A node selects the next hop such that the distance from that node to the destination is the minimum. This allows every node to select the shortest path to each destination. The distance-vector is updated at every node by a periodical dissemination of the current estimate of the shortest path to each destination.

Traditional link-state and distance-vector approaches do not scale well in large and dense MANETs [7]. A number of routing protocols have been proposed to address the problems associated with the link-state and distance-vector approaches in MANETs. These protocols can be classified into three different groups: reactive (or on-demand), proactive (or table-driven) and hybrid approaches. In reactive protocols, routes are determined until necessary through a route discovery process. In proactive approaches, nodes periodically exchange information to maintain up-to-date routing information. Hybrid routing protocols combine basic properties of both approaches.

\subsubsection{Reactive Routing Protocols}

In reactive routing protocols $[7,58,19]$, discovery and maintenance of routes are delayed until necessary. When a given node needs to send a packet to any other node in the network, the sender node initiates the process to construct a path to reach the destination. To discover a route, a node floods the route request messages through the network. When a node with a route to the destination (or the destination itself) is reached, a route replay message is sent back to the source node. Reactive protocols can be classified into two categories: source routing and hop-by-hop routing $[7,19]$.

In source on-demand protocols [7, 19], each data packet carries the complete route to destination address. Therefore, each intermediate node retransmits the packet using the information contained in its header. This means that the intermediate nodes do not need to maintain up-to-date routes to every destination. Additionally, nodes do not need to maintain neighbor connectivity. Source routing protocols do not perform well in large MANETs. The probability of failures increases proportionally to the number of intermediate nodes in each route. Furthermore, if the number of intermediate nodes grows, then 
the overhead generated by the size of the header of each data packet increases too. Therefore, in large MANETs with significant number of hops and high mobility, source routing protocols do not scale well.

In hop-by-hop (or point-to-point) routing protocols [7, 19], each data packet only carries the next hop and the destination address. All intermediate nodes use their own routing information to forward each data packet towards the destination. The main advantage of this strategy is that the routes may adapt dynamically to the changes in the network topology, since each intermediate node updates its routing table when they receive up-to-date information; this allows to compute better and fresher routes. Up-to-date routes means that less route recalculations are required during data transmission. The main drawback of this approach is that every node must store and maintain routing information for every possible destination. Nodes must be aware of their neighbors through the exchange of beaconing messages. Reactive approaches generate less traffic but experiment high latency at the beginning of the communication process. Some reactive models proposed to increase the performance in MANETs are:

- Light-Weight Mobile Routing (LMR) [35] is an on-demand routing protocol, which implements a flooding technique to determine its routes. Nodes in LMR construct and maintain multiple routes to each required destination. Therefore, when a route fails, nodes only have to select the next available route to a particular destination without initiating a route discovery procedure. A second advantage is that every node only maintains routing information to their neighbors to avoid extra delays and storage overheads associated with maintaining complete routes. However, the main drawback of the protocols is that it may generate temporary invalid routes, which introduces extra delays to determine a correct path.

- Temporally Ordered Routing Algorithm (TORA) [77] is based on the LMR [35] protocol. It uses a similar link reversal and a route repair procedure as in LMR. TORA attempts to improve scalability using a non-hierarchical routing algorithm. In its operation the algorithm attempts to suppress the generation of far-reaching control message propagation. Therefore, TORA builds and maintains a Directed Acyclic Graph (DAG) rooted at a destination. The core of the protocol is the generation of 
control messages to a small set of neighbouring nodes, where the topology change has occurred. A second advantage is that TORA can be used in conjunction with the Lightweight Adaptive Multicast algorithm (LAM) [48] to provide multicasting, however this is not par of its original design. The disadvantage of TORA is that the algorithm may also produce temporary invalid routes as in LMR.

- Dynamic Source Routing (DSR) [50], implements source routing to forward data packets. This mechanism allows to avoid path loops. To discover a route, a node floods a Route Request (RREQ) message; any receiving node adds its address to the RREQ message and retransmits the packet. Once the packet has reached its final destination, the last node reverses the route and sends a Route Reply (RREP) message if bidirectional communications is possible; otherwise, the destination node sends a RREQ messages back to the originator. An advantage of DSR is that every node also maintains a route cache with multiple routes; this avoids doing a route discovery process for already known routes. This is particularly suitable in networks with low mobility. Link breaks in the route are prevented by a mechanism of route maintenance. Another advantage is that DSR does not require any periodic beaconing, hence nodes can enter sleep mode to save energy. This also minimizes the traffic in the network.

- Ad hoc On-demand Distance Vector (AODV) [79] is a distance vector routing protocol. It is based on DSDV [80] and DSR [50] routing protocols. AODV implements the periodic beaconing and sequence numbering procedure of DSDV and RREQ and RREP messages to discover routes as in DSR. The main difference with DSR is that only the destination address is included in AODV packets, whereas DSR packets include all intermediate nodes to the destination. This change reduces the overhead in the network. A second difference is that in AODV the RREP messages only include the destination IP address and the sequence number, to avoid routing loops. AODV may adapt to highly dynamic networks. However, nodes may experience long delays during route computation; when a link fails, a new route discovery process must be initiated. This introduces extra delays and consumes more bandwidth, as the size of the network increases. 


\subsubsection{Proactive Routing Protocols}

In the proactive approach $[7,19,58]$, every node generates routing information periodically to maintain and construct routing tables even if there is no data traffic to deliver. Information contained in routing tables, is updated when the topology changes. Thus, every node maintains routing information to every other node in the network. Proactive routing protocols may use either hop-by-hop or source routing strategies to forward data traffic. The performance of the network degrades due to the exchange of control traffic messages, but the packets experience less latency because the routes are always constructed and maintained for eventual data traffic. Proactive protocols work better in networks with low mobility. Several proactive routing protocols have been proposed for MANETs; some examples are:

- Destination-Sequenced Distance Vector (DSDV) [80] is a table-driven routing protocol based on the Bellman-Ford algorithm. The main contribution of the algorithm was to solve the routing loop problem by including a sequence number in each entry in the routing table. DSDV provides a single path to a destination, which is selected using a shortest path routing algorithm. Due to its proactive nature, DSDV requires a regular update of its routing tables. In order to reduce the amount of overhead transmitted through the network, two types of update packets are used: full dump and incremental. Full dump packets carry all the available routing information and the incremental packet carries only the information changed since the last full dump. However, DSDV still introduces large amounts of overhead to the network due to the requirement of the periodic update messages. DSDV does not scale in large networks since a large portion of the network bandwidth is used for the updating procedure.

- Fish Eye Routing (FSR) [78] is a link-state routing protocol designed to improve scalability in large networks. FSR introduces the notion of multi-level scope, to minimize the overhead generated by excessive routing update messages. FSR nodes store link-state information for any other destination in the network. Every node periodically broadcasts the network with updated information of a destination to its neighbors with a frequency that depends on the distance (in hops) to that destination, 
i.e., information about distant nodes is broadcast less often. From link-state entries, nodes construct the topology map of the entire network and compute optimal routes. Every node has a precise knowledge of its local neighborhood while knowledge of distant nodes becomes less accurate.

- Topology dissemination Based on Reverse-Path Forwarding (TBRPF) [75] is a linkstate routing protocol that uses hop-by-hop routing along the shortest paths to each destination. Based on partial topology information stored in its topology table, every node uses a modified Dijkstra's algorithm to construct a source tree to reach any other node in the network. To minimize overhead, every node reports only a part of its source tree to its neighbors. TBRPF uses a combination of periodic and differential updates to keep all neighbors informed of the reported part of its source tree. Nodes have the option of reporting additional topology information (up to the full topology), to provide improved robustness in highly mobile networks.

- Wireless Routing Protocol (WRP) [71] is an improved distance-vector routing protocol designed for MANETs. It uses the Bellman-Ford algorithm to calculate paths. To avoid loops and enable faster convergence, every node reports the distance and the last hop to every destination node. Like DSDV, nodes maintain an up-to-date view of the network, every node has an available route to every destination node in the network. It differs from DSDV in table maintenance and in the update procedures. While DSDV maintains only one topology table, WRP uses a distance table, routing table, link cost table, and a message retransmission list.

- Optimized Link State Routing (OLSR) [34] is a link-state routing protocol designed exclusively for MANETs. OLSR has received particular attention. The core optimization of the protocols is the selection of Multipoint Relay (MPR) nodes as a flooding mechanism for distributing link state information. A valid MPR set, is defined as a subset of one-hop neighbors, such that all two-hop neighbors are covered through at least one node in the MPR set. The MPR-based flooding mechanism reduces the amount of control traffic information in the network. As a second optimization, only partial link state is diffused in the network. OLSR is described in detail in Chapter 2. 


\subsubsection{Hybrid Routing Protocols}

Additionally, we can find hybrid approaches that combine characteristics of both reactive and proactive routing protocols, such as:

- Zone Routing Protocol (ZRP) [42] is a hybrid routing protocol with both a proactive and a reactive routing components. $\mathrm{ZRP}$ was proposed to reduce overhead generated by the flooding of control information in proactive routing protocols and minimize the latency caused by the route discovery process in reactive routing protocols. ZRP defines a routing zone of $k$ hops around every node. A proactive strategy, Intrazone Routing Protocol (IARP) is used inside routing zones, and a reactive strategy, Inter-zone Routing Protocol (IERP), is used between routing zones. A route to a destination within the local zone is constructed by using the information from the routing table by IARP. Therefore, if source and destination are in the same zone, packets can be delivered immediately. For routes beyond the local zone, route discovery happens reactively (i.e., implementing RREQ and RREP messages).

- Zone-based Hierarchical Link State (ZHLS) [49] is a hierarchical routing protocol designed for MANETs. ZHLS divides the network into non-overlapping zones. ZHLS defines two levels of topologies: node level and zone level. A node level topology shows how the nodes of a zone are physically connected to one other. There is a virtual link between two zones if at least one node in a zone is physically linked to some nodes of the other zone. Every node constructs two routing tables, an intrazone routing table and an inter-zone routing table, by flooding Node Link-State Packets (NodeLSPs) within the zone and Zone LSPs (ZoneLSPs) throughout the network. However, this incurs in a large communication overhead in the network.

In [58], Lang evaluates and classifies in his PH.D. thesis, different routing protocols for MANETs. Also, a complete classification of routing protocol for MANETs is presented in detail by Abolhasan et al. in [7]. 


\subsection{Security issues in MANETs}

Wireless networks are more vulnerable to attacks. Unlike wired networks, data travel across the air. On a wireless network, an intruder can easily eavesdrop on all data transmissions within its range by operating in promiscuous mode. This can happen even without the sender know that is under attack. Additionally, due to the limitations of the medium, communication may be perturbed, e.g., jamming communications with noise. The routing protocols presented in the previous section are not secure by design. An intruder may disobey the rules of the protocol to control the data traffic to disrupt the communication or to perturb the network topology. Also, a node may misbehave unintentionally, e.g., node malfunction, energy lost or radio interference. In [99], Wu et al., present a survey of attacks in MANETs. The authors classify the attacks in passive and active attacks. In the context of this thesis, a malicious node is defined as a node that interrupts the flooding of control traffic information or does not obey the rules of the protocol. The terms: malicious, misbehaving, attacker and intruder are equivalent.

\subsubsection{Passive Attacks}

In a passive attack $[99,83]$, the data communication is intercepted without disrupting the network communication, for instance:

- Eavesdropping: Eavesdropping is the intercepting of messages or conversations by unintended receivers [99]. In a MANET, nodes share a wireless medium. In wireless communication the nodes use the RF spectrum and broadcast over airwaves that can be intercepted by intruders tuned to the same frequency.

- Traffic Analysis: In this attack, the intruder intercepts and examines wireless transmissions in order to find patterns and deduce some information from the messages being exchanged. The intruder may also store the messages intercepted for use in the future. In general, the greater the number of messages intercepted, the more can be inferred from the data traffic.

- Monitoring: In this attack, the intruder analyzes information from the network to predict the behavior of the nodes and their communication patters, e.g., mobility 
patterns or the frequency with which a node broadcasts data packets to its neighbors.

\subsubsection{Active Attacks}

In an active attack [99, 83], the intruder attempts to disrupt the network connectivity. Some examples of active attacks are:

- Cache poisoning. In this attack, the intruder overhears data packets and includes in its cache incorrect routing information from that packet. Afterwards, the intruder may send packets spoofing other nodes identities or links to non-neighbor nodes.

- Denial of Service (DoS). In this attack, the intruder may overflow the network with a storm of messages to saturate the medium. In a variation of the attack, the intruder can generate invalid messages to keep other nodes busy (waisting their CPU cycles and draining their battery power). As a consequence, the network functioning and communication are disrupted.

- Replay attack. In this attack, the intruder records old valid control messages and re-sends them to make other nodes obtain information about a network topology that no longer exists. The attack may succeed when control messages carry a digital signature that does not include a timestamp.

- Blackhole attack [67]. In this attack, the intruder drops (in bulk or selectively) received control traffic messages instead of relaying them according to the rules of the protocol. In a variation of the attack, the intruder may selectively forward control information, i.e., to drop routing information for a specific node, a packet every $n$ packets, or a packet every $t$ seconds. This reduces the amount of available routing information to other nodes.

- Message Tampering. In this attack, the intruder modifies the messages originating from other nodes before retransmitting them. The attack is successful if a mechanism for message integrity is not implemented (e.g., a digest of the payload).

- Wormhole attack. In this attack, the intruder records packets from one region of the network and tunnels them to another region. Two intruders connected by a wireless 
or a wired private medium may collude to establish a longer wormhole. Routing is affected when control traffic information is tunneled without following the rules of the routing protocol. The attack is very difficult to detect even in networks where integrity, confidentiality, non-repudiation and authentication are preserved via encryption, access control mechanisms or digital signature.

\subsection{Summary of Contributions}

The OLSR protocol has received particular attention. OLSR is defined in RFC 3626 [34]. As many other routing protocols for MANETs, OLSR is not secure by design. The selection of the MPRs and exchange of topology control information are important vulnerability targets. Therefore, we use OLSR to show vulnerabilities in link-state routing protocols for MANETs and to implement our proposed countermeasures. This security risks and proposed solution can be adapted to other proactive (or reactive) routing protocols for MANETs.

Our first contribution deals with the effect of control traffic attacks in OLSR networks and the selection of MPR sets with additional coverage to mitigate their effect [23]. The MPR selection with additional coverage is presented in RFC 3626 [34]; we name it $k$ Covered-MPR selection. However, additional coverage reduces the performance of the network due to additional control traffic information, i.e., Topology Control (TC) messages. We propose a $k$-Robust-MPR selection. In a $k$-Robust-MPR selection a node selects, when possible, $k+1$ disjoint MPR sets to guarantee that even if $k$ of the selected MPR sets become invalid, the remaining set is still a valid MPR set. Our proposed MPR selection offers equivalent protection against control traffic attacks also reducing the overhead generated by additional control traffic information. As part of our contribution, we present in Chapter 3 a taxonomy of flooding disruption attacks in link-state routing protocols for MANETs

As a second contribution, we present the effect of flooding disruption attacks in HOLSR 
networks [24]. HOLSR uses TC messages for intra-cluster communications and implements Hierarchical TC (HTC) messages for inter-cluster communications. HOLSR implements the MPR flooding mechanism for distributing control traffic information. HTC messages are flooded exclusively by the MPRs. Therefore, the inter-cluster communications are also affected by flooding disruption attacks. In Chapter 6, we propose to mitigate the effect of the attacks against HTC messages by selecting MPR sets with additional coverage (i.e., $k$-Robust-MPR and $k$-Covered-MPR selections).

Additionally, the cluster formation phase in hierarchical OLSR networks can be disturbed. Our algorithm HASH-CHAINED_CID_DISSEMINATION (HCCD) [25] is proposed to enforce the cluster formation phase in HOLSR networks. In HOLSR, Cluster ID Announcement (CID) messages are implemented to organize the network in clusters. A misbehaving node may maliciously alter mutable fields (e.g., hop count) in CID messages to unbalance the distribution of nodes in clusters. Our solution is based on hash chains and allows a node to detect and discard invalid CID messages. Our algorithm can be implemented in other hierarchical approaches that use messages with mutable fields to organize the network in clusters. $\mathrm{HCCD}_{2}$ is a variation of the HCCD algorithm to handle a misbehaving node with more than one interface that tunnels valid CID messages between cluster at different levels.

Finally, in Chapter 7, we analyze vulnerabilities in multipath OLSR-based networks. We propose a Disjoint Multipath OLSR (DM-OLSR) strategy to compute strictly disjoint paths. MP-OLSR [103, 104, 105, 102], proposed by Yi et al., is based on the MPR flooding mechanism to distribute control traffic information in the network. The construction of multiple paths in MP-OLSR has two phases: topology discovery and route computation. In the first phase, the nodes obtain information about the network topology through the exchange of Hello and TC messages. In the second phase, the nodes compute multiple paths to a particular destination in the network based on the information gathered during the first phase. These two phases are affected by flooding disruption attacks. Additionally, MPRs report partial link state information. Therefore, MP-OLSR nodes only acquire a 
partial view of the network. We analyze how the construction of multiple paths in MPOLSR networks is affected by flooding disruption attacks and incomplete view of the network topology. In MP-OLSR redundant information in the TC messages is proposed to increase the chances of discovering paths. However, their size increases considerably. This reduces the performance of the network and in some situations is not enough to mitigate the effect of flooding disruption attacks. Our strategy relies on MPR sets with additional coverage to enhance the network discovery and the route computation phases.

\subsubsection{Structure of the thesis}

In Chapter 2, we describe OLSR in detail. Security issues in OLSR networks are reviewed in Chapter 3. In Chapter 4 we review existing solutions and related work. In Chapter 5, we review the effect of control traffic attacks in OLSR networks and our proposed countermeasures. In Chapter 6, we review the effect of flooding disruption attacks in hierarchical OLSR-based networks. Chapter 7 presents security issues in multipath OLSR networks and proposed countermeasures. Chapter 8 , presents our conclusions and future work. 


\subsubsection{Publications}

This thesis is based on the following publications each representing different aspects of the thesis.

\section{Book Chapters}

- "Security issues in link state routing protocols for MANETs". G. Cervera, M. Barbeau, J. Garcia-Alfaro and E. Kranakis. Advances in Network Analysis and Applications, Proceedings of MITACS Workshops. Springer Series on Mathematics in Industry. Evangelos Kranakis, ed., expected publication date July 2012.

\section{Refereed Conference Papers}

- "Mitigation of topology control attacks in OLSR networks". G. Cervera, M. Barbeau, J. Garcia-Alfaro, and E. Kranakis. In 5th International Conference on Risks and Security of Internet and Systems (CRISIS), Jean-Marc Robert, editor, pages 81-88, Montreal, Canada, October 10-13, 2010.

- "Mitigation of flooding disruption attacks in HOLSR networks". G. Cervera, M. Barbeau, J. Garcia-Alfaro, and E. Kranakis. In 9th Annual Conference on Communication Networks and Services Research Conference (CNSR), pages 167-174, 10.1109/CNSR.2011.32. Ottawa, ON, Canada, May 2-5, 2011.

- "Preventing the cluster formation attack against the hierarchical OLSR protocol: Invited Talk". In 4th Canada-France MITACS Workshop on Foundations and Practice of Security (FPS), Paris, France, May 12-13, 2011. Springer LNCS.

\section{Journal Paper}

- "A Multipath routing strategy to prevent flooding disruption attacks in link state routing protocols for MANETs"'. G. Cervera, M. Barbeaua, J. Garcia-Alfaro and E. Kranakis. To be submitted. 


\section{Chapter 2}

\section{Optimized Link State Routing Protocol}

\subsection{Introduction}

This chapter presents an overview of the OLSR [34] protocol. OLSR is a proactive routing protocol designed for MANETs. The core of the protocol is the selection, by every node, of Multipoint Relays (MPRs) among their one-hop neighbors. A valid MPR set, is defined as a subset of one-hop neighbors, such that all two-hop neighbors are covered through at least one node in the MPR set. Unlike other link state routing protocols (e.g., OSPF [69]), the MPRs report partial link state information. Therefore, the MPR mechanism reduces the size and amount of control traffic information flooded in the network. Fig. 2.1 compares the MPR mechanism and classical flooding. In Fig. 2.1(a), control traffic information is retransmitted by all the one-hop neighbors. In Fig. 2.1(b), control traffic information is retransmitted exclusively by the MPRs.

OLSR is defined in RFC3626 [34]. A second version of the protocol, i.e., OLSRv2, is presented by Clausen et al. in an Internet-Draft [30]. OLSRv2 uses and extends: the MANET Neighbor Discovery Protocol (NHDP) [28], RFC5444 - Generalized MANET Packet/Message Format [29], RFC5497 - Representing Multi-Value Time in MANETs [26] and RFC5148 - Jitter Considerations in MANETs [27] (optional). These protocols were all originally created as parts of OLSRv2, but have been specified separately for wider use. OLSRv2 retains the same basic mechanisms and algorithms for distributing control traffic (i.e., MPR-based flooding) but provides a more efficient signaling framework and implements some message simplifications.

Several OLSR implementations have been developed: OOLSR (the original, object-oriented implementation of OLSR by INRIA HIPERCOM) [1], NLROLSRd (by the U.S. Naval Research Laboratory) [4], NOA-OLSR (by Niigata University) [3], OLSR11 win (by the 


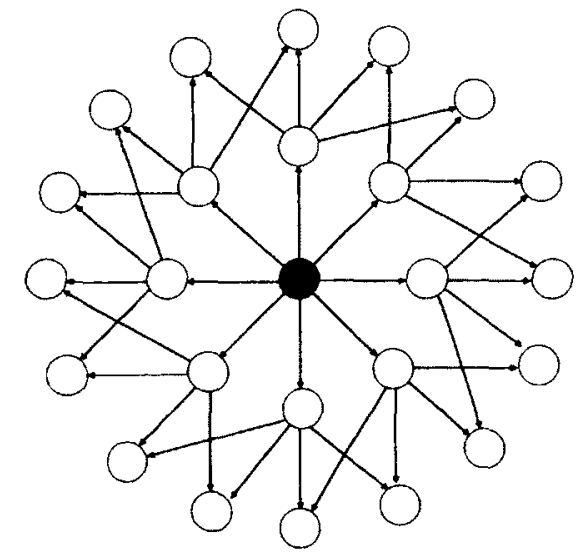

(a) Classical flooding.

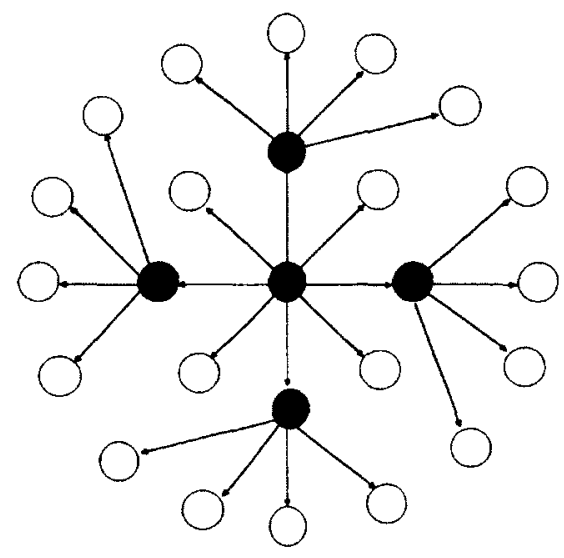

(b) MPR mechanism.

Figure 2.1: MPR based mechanism against the classical flooding. Consider gray nodes as the originators of a TC message and black nodes as MPRs.

GRC, Universitad Politécnica de Valencia), the OLSRd (OLSR daemon by UniK, University of Oslo) [5], UM-OLSR (by the University of Murcia) [87] and CRC OLSR (by the Communication Research Centre in Canada) [36]. Furthermore, multiple extensions of the protocol have been proposed to address different routing problems in MANETs. For instance, HOLSR (Hierarchical OLSR) [96] has been proposed to solve scalability problems, MPOLSR(Multipath OLSR) [102] to improve QoS and fault tolerance, and SMOLSR (Simple Multicast OLSR) [2] for multicast routing in MANETs. In the following section we present the OLSR protocol in detail.

\subsection{Neighbor Discovery Process}

OLSR nodes flood the network with link state information messages. The link state information is constructed by every node and involves periodically sending Hello and Topology Control (TC) messages. This information is used to determine the best path to every destination in the network. Due to the proactive nature, the routes are immediately available when needed. The OLSR protocol is based on hop by hop routing, i.e., each routing table lists, for each reachable destination, the address of the next node along the path to that 
destination. To construct a topology map, every node implements a topology discovery mechanism leveraging the periodic exchange of control traffic messages. Topology discovery includes: link sensing, neighbor detection and topology sensing. In the first phase, every node populates its local link information base (link set) and establishes communication with their symmetric neighbors, i.e., nodes with bidirectional communication. This phase is exclusively concerned with the OLSR interface addresses and ability to exchange packets between such OLSR interfaces.

During the neighbor detection phase, every node populates its neighborhood information base (i.e., one-hop and two-hop neighbor set). The link sensing and neighbor detection phases are based on the periodic exchange of Hello messages. Hello messages are solely transmitted to one-hop neighbors. In every Hello message, the nodes report their one-hop neighbors. This information allows every node to construct and maintain a neighbor set, as well as to select its MPR set. In the neighbor set, every node records the information about the one-hop neighbor link status (i.e., unidirectional, bidirectional or MPR), with this information every node builds its MPR selector set, i.e., the neighbors that selected that node as their MPR. OLSR detects and eliminates duplicate messages. OLSR keeps track of recently received messages by using a duplicate table. Therefore, when a message has been received and included in the duplicate table, the payload is not examined and the message is automatically discarded.

\subsection{MPR Computation}

The RFC 3626 [34] proposes an heuristic for selection of MPRs. A node selects its MPR set such that every node two-hops away is covered by at least one node in the MPR set. This MPR selection is applied per interface. The MPR set for a node is the union of the MPR sets constructed for each interface. In every Hello message, the nodes advertise their willingness to forward traffic on behalf other nodes. A node must advertise the same willingness on all interfaces. The possible values are:

- WILL_NEVER: the node must not be selected as MPR. 
- WILL_ALWAYS: the node should be selected as MPR.

- WILL_DEFAULT: is the default value.

The proposed heuristic in RFC3626 [34] to compute an MPR set for a given node $n$, is as follows:

1. Select all one-hop neighbor nodes with willingness equal to WILL_ALWAYS.

2. Compute the degree of every one hop neighbor. The degree of a one-hop neighbor $n_{1}$ is defined as the number of symmetric neighbors of node $n_{1}$ excluding the one-hop neighbors of node $n$ and the node itself.

3. Include in the MPR set every one-hop neighbor which is the "only" node to provide reachability to a node two-hops away. Remove all two-hop neighbor nodes which are now covered by at least one node in the MPR set.

4. While there exist two-hop neighbor nodes which are not yet covered by at least one node in the MPR set, then:

(a) For every one-hop neighbor, calculate its reachability, i.e., the number of twohop neighbor nodes which are not yet covered by at least one node in the MPR set, and which are reachable through this one-hop neighbor;

(b) Select as a MPR the node with highest willingness among the one-hop neighbor nodes with non-zero reachability. In case of multiple choice select the node which provides reachability to the maximum number of two-hop neighbors. In case of multiple nodes providing the same amount of reachability, select the node with highest degree. Remove the two-hop neighbor nodes which are now covered by a node in the MPR set.

5. The MPR set is generated from the union of the MPR sets for each interface.

\subsection{Network Topology Discovery}

Topology sensing is achieved through the exchange of TC messages. TC messages are generated and retransmitted exclusively by the MPRs. TC messages have a Time-to-Live 
(TTL) field that is decremented every time an MPR retransmits the message. These messages allow each node to construct its topology set and to declare its MPR selector set. A TC message contains the MPR selector set of its originator. A node that has an empty MPR selector set does not send or retransmit any TC message. An MPR forwards a message only if it comes from a node in its MPR selector set (i.e., a source-dependant mechanism). This forwarding algorithm is defined in RFC 3626 [34]. Using the information from TC messages, each node maintains a topology set where each entry consists of:

- an identifier of a possible destination, i.e., an MPR selector in a TC message,

- an identifier of a last-hop node to that destination, i.e., the originator of the TC message, and

- an MPR selector set sequence number [47].

It implies that a possible destination (i.e., an MPR selector) can be reached through the originator of the TC message. If there is an entry in the topology set whose last-hop address corresponds to the originator of a new TC message and the MPR selector set sequence number is greater than the sequence number in the received message, then the new message is discarded. Routing tables are constructed using the information from the onehop neighbor, two-hop neighbor and topology sets.

OLSR implements two optional messages: Multiple Interface Declaration (MID) and Host and Network Association (HNA). They are exclusively retransmitted by the MPRs following the default forwarding algorithm defined in RFC 3626 [34]. MID messages are used to declare the presence of multiple interfaces on a node. HNA messages are employed to inject external routing information into the OLSR-based network and provide connectivity to nodes with non-OLSR interfaces (e.g., Internet). MID messages are implemented

Table 2.1: Summary of control traffic messages in OLSR. MID and HNA messages are optional.

\begin{tabular}{llll}
\hline Messages & Generated by & Forwarded by & Reported information \\
\hline Hello & Every node & N/A & One-hop neighbors \\
TC & MPRs & MPRs & MPR Selector Set \\
MID & Nodes with multiple interfaces & MPRs & All available interfaces \\
HNA & Nodes with external access & MPRs & External routing information \\
\hline
\end{tabular}


in a network with multiple interface nodes. Additional information is necessary in order to map interface addresses to main addresses. In OLSR, the main address is defined as the OLSR interface address. A node with multiple interfaces must generate periodically MID messages announcing all its interfaces to other nodes in the network. Thus, every node in an OLSR network will associate multiple interfaces to a node's main address. Nodes with just one interface do not generate MID messages and their main address is the OLSR interface address. A node with several interfaces, where only one of them is participating in an OLSR-based network must not generate MID messages. Upon receiving a MID message, the information is stored in an Interface Association set. This information is used to construct the routing tables. When a node misbehaves and does not retransmit TC, HNA or MID messages, the proper construction of the routing tables is compromised. Table 2.1 presents all the messages implemented in OLSR.

\subsection{Format of Packets and Messages in OLSR}

In OLSR, several type messages can be transmitted in one packet. The basic layout of any packet in OLSR is showed in Fig. 2.2. In the figure, the IP and UDP headers are omitted. The packet header has two main fields:

\subsubsection{Packet and Message Header}

The basic layout of an OLSR packet is shown in Fig. 2.2 (the IP and UDP headers are omitted). The fields are:

- Packet Length. The length (in bytes) of the packet. If the packet length is less than or equal to the size of the packet header, then the message is discarded.

- Packet Sequence Number (PSN). The PSN is incremented by one each time the packet is forwarded. Sequence numbers are used with the purpose of discarding old information.

The format of the message header proposed in RFC 3626 [34] includes the fields:

- Message Type. This field indicates the type of message that is included in the packet, e.g., Hello, TC, etc. Different types of messages can be transmitted in one packet. 
1

2

3

01234567890123456789012345678901

\begin{tabular}{|l|c|c|}
\hline \multicolumn{2}{|c|}{ Packet Length } & Packet Sequence Number \\
\hline Message Type & Vtime & Message Size \\
\hline Time to Live & Hop Count & Message Sequence Number \\
\hline \multicolumn{3}{|c|}{ Message } \\
\hline Message Type & Vtime & Message Size \\
\hline \multicolumn{3}{|c|}{ Originator Address } \\
\hline Time to Live & Hop Count & Message Sequence Number \\
\hline & \\
\end{tabular}

Figure 2.2: Example of an OLSR packet.

- Vtime. Indicates for how long the information contained in the message must be considered valid by the receiver.

- Message Size. This field indicates the size in bytes of the message measured from the beginning of the Message Type field until the beginning of the next message or the end of the packet if there are no more messages.

- Originator Address. This field contains the main address of the node which has originally generated the message. This field must never be changed in retransmissions. The Source Address field from the IP header is changed each time the packet is forwarded.

- Time to Live(TTL). This field indicates the maximum number of hops a message will be retransmitted. The TTL value is decremented by one for each time a message is retransmitted. 
- Hop Count. This field shows the number of times a messages has been retransmitted. Before a message is retransmitted the field is incremented by one.

- Message Sequence Number. This is a unique identification number assigned by the originator of the message. The sequence number is increased by one for each message the node generates. This field allow the receivers to discard future copies of previously received messages.

\subsubsection{Hello Message Format}

The Hello message format is prepared to accommodate for link sensing, neighborhood detection and MPR selection signaling or future extensions. Fig. 2.3, shows the format of Hello messages. The format proposed in RFC3626 [34] includes the fields:

- Reserved. This field has to be initialized to "000000000000000".

- HTime. This field specifies the Hello emission interval used by the sender.

- Willingness. This field specifies the willingness of the sender to carry and forward traffic on behalf other nodes.

- Link Code. This fields specifies information about the status of the neighbor (Neighbor Type) and the link (Link Type) between the interface of the sender and the following list of neighbor interfaces. The following "Link Types" are defined in OLSR:

- UNSPEC_LINK: this link type indicates that there is no information about the links.

- ASYM_LINK: this link type indicates that a node has been detected but the link is asymmetric.

- SYM_LINK: this link type indicates that the node has a symmetric link and can be considered as a neighbor.

- LOST_LINK: this link type indicates that the links have been lost.

The following "Neighbor Type" are defined by OLSR: 


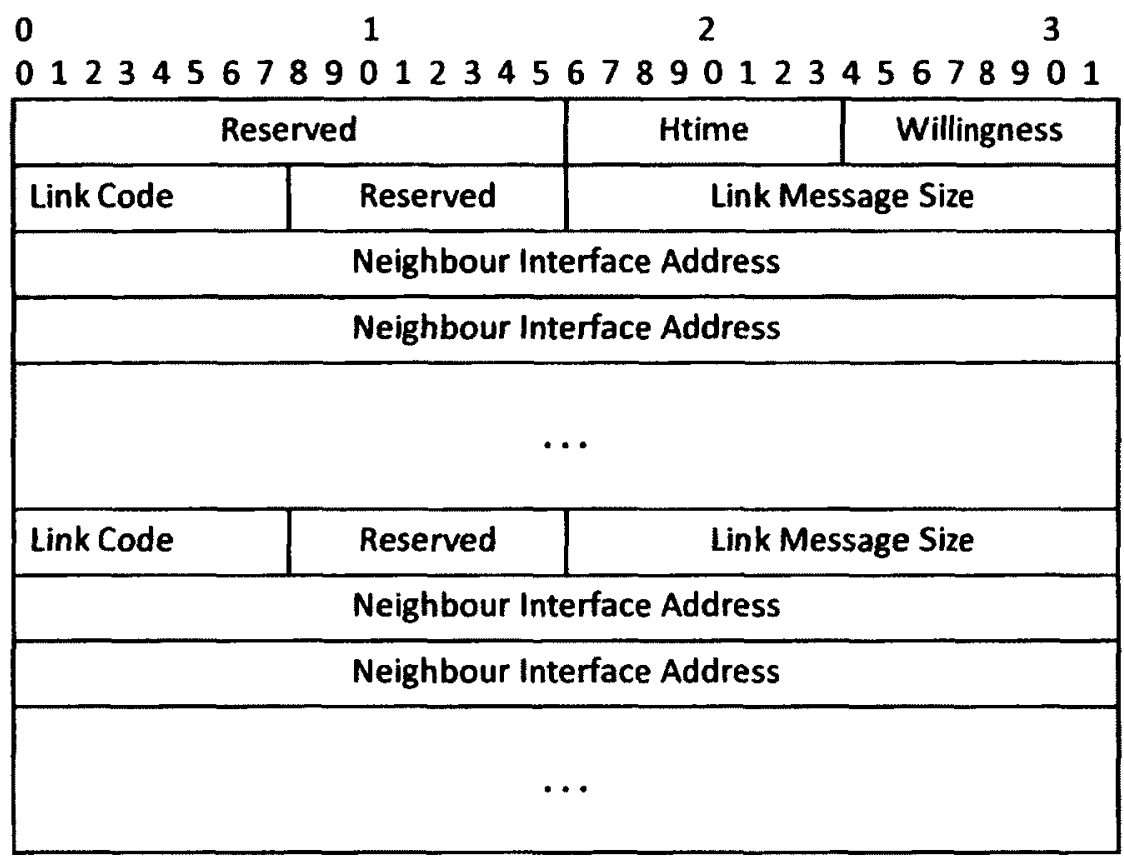

Figure 2.3: Example of a Hello message.

- SYM_NEIGHBOR: this neighbor type indicates that the neighbors are symmetric, i.e., they have at least one symmetrical link.

- MPR_NEIGH: this neighbor type indicates that the neighbors are symmetric and have been selected as MPR by the sender.

- NOT_NEIGH: this neighbor type indicates that the nodes are not longer or not yet become symmetric neighbors.

- Link Message Size. This field specifies the size of the link message (in bytes) measured from the beginning of the "Link Code" field until the next "Link Code" field (or the end of the message if there is no more link types).

- Neighbor Interface Address. This field contains the address of an interface of a neighbor node. 


\begin{tabular}{|c|c|}
\hline ANS & Reserved \\
\hline \multicolumn{2}{|c|}{ Advertised Neighbour Main Address } \\
\hline \multicolumn{2}{|c|}{ Advertised Neighbour Main Address } \\
\hline
\end{tabular}

Figure 2.4: Example of a TC message.

\subsubsection{TC Message Format}

The TC message format is prepared to accommodate for neighbor nodes, i.e., selector nodes. Fig. 2.4, shows the format of TC messages. The format proposed in RFC3626 [34] includes the fields:

- Advertised Neighbor Sequence Number (ANSN). This field includes a number associated with the advertised neighbor set. Every time a node detects a change in its advertised neighbor set, it increments this sequence number. This allows to detect and discard previously received messages.

- Advertised Neighbor Main Address. This field includes the main address of the advertised neighbor.

\subsubsection{MID and HNA Message Format}

The MID message format is prepared to accommodate for neighbor interface addresses. Fig. 2.5(a), shows the format of MID messages. The format proposed in RFC3626 [34] includes the field:

- OLSR interface address. This field contains the address of an OLSR interface node. The nodes main address is not included but it is indicated in the originator of the message. 


\begin{tabular}{c}
0 \\
01234567890123456789012345678901 \\
\hline OLSR Interface Address \\
\hline OLSR Interface Address \\
\hline OLSR Interface Address \\
$\ldots$ \\
\hline
\end{tabular}

(a) Format of a MID message.

\begin{tabular}{c} 
1 \\
01234567890123456789012345678901 \\
\hline Network Address \\
\hline Netmask \\
\hline Network Address \\
\hline Netmask \\
\hline
\end{tabular}

(b) Format of a HNA message.

Figure 2.5: Optional OLSR messages.

The HNA message format is presented in Fig. 2.5(b). The format proposed in RFC3626 [34] includes the fields:

- Network Address. This field contains the network address of the associated network.

- NetMask. This field contains the netmask of the network address immediately above. 


\subsection{Routing Table Calculation}

In OLSR, every node maintains a routing table which allows it to route data to any other node in the network. The routing table is based on the information contained in the local link information base and the topology set. A shortest path algorithm e.g., Dijkstra's algorithm, is used to compute optimal routes to every destination. The routing table is calculated when a change is detected in any of these sets:

- the Link set,

- the One-hop Neighbor set,

- the Two-op Neighbor set,

- the Topology set,

- the Multiple Interface Association set, or

- the Host and Network Association Information set.

Therefore, if a change is detected in any of this sets, then the routing table is recalculated to update the routing information to every known destination in the network. All the destinations, for which a route is broken or only partially known, are not recorded in the routing table. Each entry in the routing table consists of:

- Destination Address: Identifies the address of the destination node.

- Next Address: Contains the address of the next node (i.e., one-hop neighbor) to reach the destination node.

- Distance: The distance in hops, to reach the destination node.

- Interface Address: The local interface to reach the destination node.

The routing table is always recalculated in case of neighbor detection or loss, when a two-hop neighbor is detected or removed, when a topology tuple is created or removed, or when the multiple interface association information changes. 


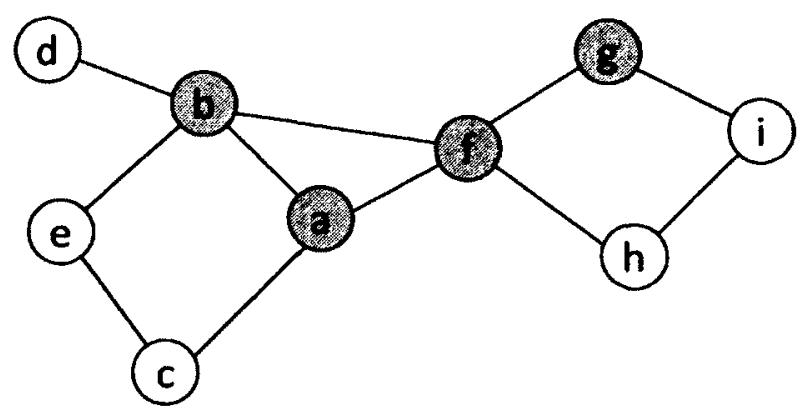

\begin{tabular}{ll}
\hline MPRs & Selector Set \\
\hline$a$ & $b, c, f$ \\
\hline$b$ & $a, e, f, d$ \\
\hline$f$ & $a, b, h, g$ \\
\hline$g$ & $f, i$ \\
\hline
\end{tabular}

Figure 2.6: Example of an OLSR-based network.

\subsection{Example of an OLSR-based network}

In summary, the network topology discovery process is performed as follows:

1. First, every node periodically generates Hello messages to advertise itself and establish bidirectional links with its one-hop neighbors. Hello messages are not retransmitted. Fig. 2.6 shows an example of an OLSR-based network. Node $a$ includes nodes $b, c$ and $f$ in its one-hop neighbor set after exchanging Hello messages and establishing bidirectional links.

2. In subsequent Hello messages, every node reports its one-hop neighborhood. Receiver nodes identify their two-hop neighbors and compute their MPR set. In Fig. 2.6, nodes $d, e, g$ and $h$ are included in node $a$ 's two-hop neighbor table. Node $a$ selects nodes $b$ and $f$ as its MPRs. Nodes $a, b, f$ and $g$ are selected as MPRs.

3. Nodes report their MPR set within their following Hello messages. If the receiver node was selected as an MPR, then it includes the sender node in its selector set, e.g., node $b$ includes $a$ in its selector set.

4. Nodes with a non empty selector set periodically generate TC messages advertising all nodes within their selector set. TC messages are retransmitted exclusively by the MPRs. To reach nodes more than two hops away, node $a$ depends on the TC messages generated by all the MPRs. For instance, node $g$ must periodically generate 
TC messages advertising its selector set, i.e., nodes $f$ and $i$. TC messages generated by node $g$ are retransmitted exclusively by nodes $f, a$ and $b$.

5. When a node receives a TC message, it includes the contained information in its topology set. In Fig. 2.6, after receiving TC messages from node $g$, node $a$ identifies node $g$ as the last hop to reach node $i$. Note that node $b$ receives TC messages from nodes $a$ and $f$. However, node $b$ stores the recently received TC messages in its duplicate table and discards future copies of the same message.

6. Finally, routing tables are constructed using information from the one-hop and twohop neighbors and the topology set. Every node executes the Dijkstra's algorithm to obtain the shortest path to every other node more than two hops away. For instance, to reach node $i$, node $a$ constructs a path trough nodes $f$ and $g$. The shortest path to reach every other node in the network is always composed by MPRs. For example, to reach node $d$, node $i$ constructs a path composed by nodes $g, f$ and $b$.

7. Routing tables include the next node and number of hops to reach every other node in the network. Node $i$ stores in its routing table only the next hop to reach node $d$ (i.e., node $g$ ) and the number of hops (i.e., four hops). Thanks to the MPR mechanism, the nodes are aware of every other node in the network but some links are never advertised. For instance, node $a$ never receives information about the link between nodes $h$ and $i$, or between nodes $e$ and $c$.

8. Optionally, a node with more than one interface generates MID messages. A node with access to an external network generates HNA messages. Information contained in MID and HNA messages is loaded in routing tables.

\subsection{OLSR Security Considerations}

OLSR is not secure by design. In RFC 3626 [34], Section 20, some security considerations are proposed for confidentiality, integrity and interaction with external routing domains. Countermeasures against possible vulnerabilities are not considered. In the following section, we present security risk in OLSR-based networks. 


\section{Chapter 3}

\section{Security Issues in OLSR}

\subsection{Security Issues in OLSR}

In this section, we review vulnerabilities in OLSR and proposed countermeasures. According to Herberg and Clausen [31], in OLSR every node must acquire and maintain a routing table that effectively reflects the network topology. The routing tables constructed by every node must converge, i.e., all nodes must have an identical topology map. Therefore, the target of a misbehaving node may be that the nodes in the network (a) build inconsistent routing tables that do not reflect the accurate network topology, or (b) acquire an incomplete topology map. In the former case, the attacker may launch several types of attacks to accomplish its goal, for example:

- Identity spoofing. A misbehaving node may generate false Hello or TC messages pretending to be a different node. The attack can be launched as follows:

- A misbehaving node generates a Hello messages with a false identity. For instance, in Fig. 3.1(a), node $M_{1}$ may generate Hello messages pretending to be node $e$. As a result, the MPRs of $M_{1}$ will present themselves as the last hop to reach node $e$.

- A misbehaving generates TC messages with a false identity. For instance, $M_{1}$ may generate a TC message pretending to be node $f$ advertising node $i$ as part of its Selector Set. As a consequence, node $f$ appears to be the last hop to reach node $i$.

- Link spoofing. A misbehaving node may generate Hello or TC messages including false links to other nodes in the network. The attack can be launched as follows:

- In Fig. 3.1(a), node $M_{1}$ generates an incorrect Hello message announcing node 


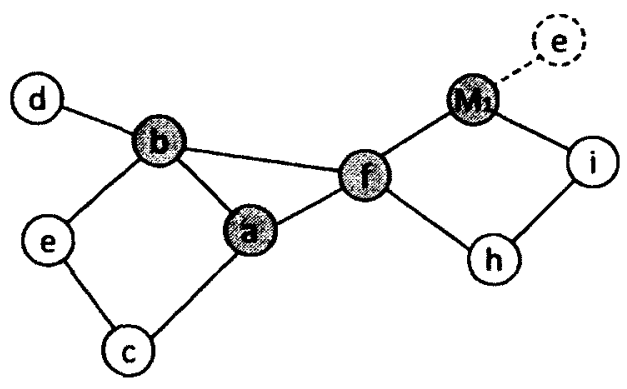

(a)

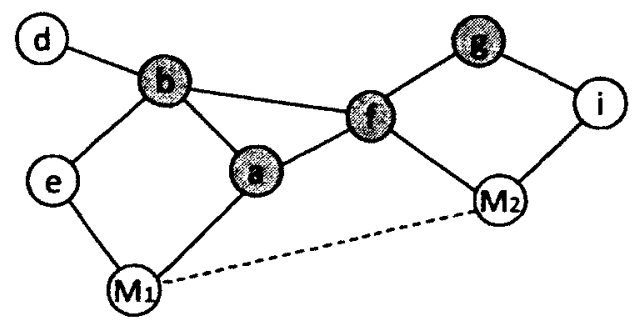

(b)

Figure 3.1: Example of an OLSR-based network with misbehaving nodes $M_{1}$ and $M_{2}$.

$e$ as its one-hop neighbor. As a result, nodes $i$ and $f$ include node $e$ in their two-hop neighbor table.

- In Fig. 3.1(a), node $M_{1}$ may also generate TC messages announcing node $e$ as part of its Selector Set. As a consequence, node $M_{1}$ appears to be the last hop to reach node $e$.

- Replay attack. In this attack, a misbehaving node resends old valid TC or Hello messages. For instance, suppose that in Fig. 3.1(a), node $M_{1}$ had a valid link to node $e$. Node $M_{1}$ may resend an outdated Hello message announcing node $e$ as its onehop neighbor even if node $e$ has moved and is not part of its one-hop neighborhood anymore. As a result, the network is flooded with stale information.

- Wormhole attack. In a wormhole attack, an inexistent link can be created by one or more nodes by tunneling valid Hello messages without following the rules of the protocol. For instance, in Fig. 3.1(b), node $M_{1}$ retransmits Hello messages between nodes $a$ and $e$. Thus, node $e$ and $a$ exchange Hello messages and establish an incorrect bidirectional link. A larger wormhole can be mounted when two misbehaving nodes collude. For instance, in Fig. 3.1(b), there exists a link between nodes $M_{1}$ and $M_{2}$ that is never reported. Nodes $e$ and $i$ exchange Hello messages through the tunnel created by nodes $M_{1}$ and $M_{2}$. As a result, nodes $e$ and $i$ establish an incorrect link. In both cases, once the incorrect link has been established, other control traffic 


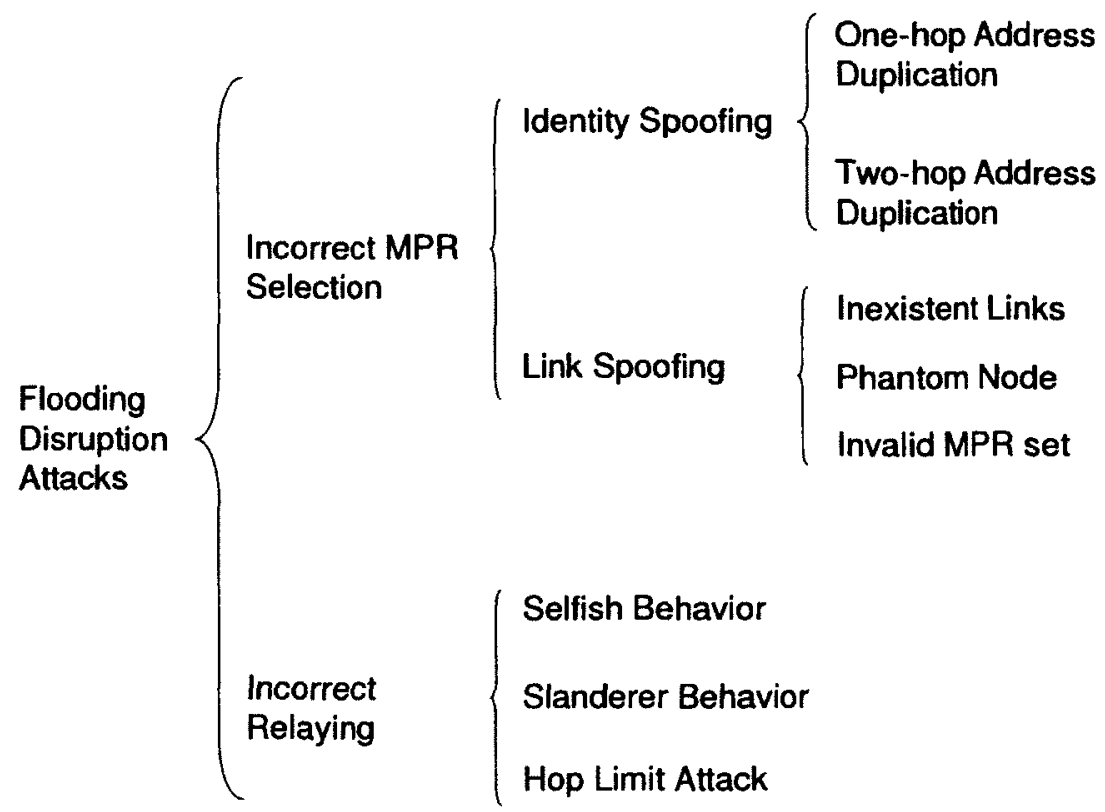

Figure 3.2: Taxonomy of flooding disruption attacks [24].

messages (i.e., TC, MID or HNA) can be tunneled.

\subsection{Flooding Disruption Attacks}

In this section, we describe flooding disruption attacks in OLSR. In the attacks presented in Section 3.1, the attacker attempts to inject incorrect information to the network. As a consequence, the nodes in the network compute inconsistent routing tables that do not reflect accurately the network topology. In link state routing protocols for MANETs, some attacks can be launched even in networks with either cryptographic capabilities [24, 32, 31] or Intrusion Detection Systems (IDS) $[65,21,46]$ implemented, e.g., a misbehaving node refuses to compute a valid MPR set.

The exchange of control traffic information and the MPR selection process are important vulnerability targets. We focus on flooding disruption attacks [24], Fig. 3.2. In this kind of attacks, the target of an attacker is to disrupt the topology map acquisition process by disturbing the flooding of valid control traffic information. In [24], we presented 
a taxonomy of these attacks and countermeasures based on the selection of the MPR sets with additional coverage. The taxonomy we presented in [24] divides the attacks in two categories:

- Incorrect MPR Selection: in this category, the malicious node either selects an incomplete MPR set or forces other nodes to compute an incorrect MPR set. To launch the attack, the malicious node may either generate control traffic information with a false identity (i.e., identity spoofing) or report inexistent links to other nodes (i.e., link spoofing). As a consequence, the affected node computes an invalid MPR set, i.e., some of its two-hop neighbors are not covered through at least one node in its MPR set.

- Incorrect Relaying: in this category, the malicious node does not generate control traffic information (i.e., TC, MID or HNA messages) or does not forward valid messages on behalf of other nodes, e.g., selfish attack. In a variation of the attack, a malicious node may report incomplete information or eliminate some information reported by other nodes, e.g., slanderer behavior. Additionally, the misbehaving node can maliciously alter mutable fields in the messages before forwarding them, e.g., hop limit attack.

Fig. 3.2 summarizes flooding disruption attacks in OLSR and the mechanisms used to perform them. In the sequel, we present these security threats in more detail.

\subsubsection{Incorrect MPR Selection}

In this section, we describe vulnerabilities against the MPR selection process and some techniques to launch the attacks, i.e., link or identity spoofing.

Identity Spoofing. The identity spoofing attack [31] is performed by a malicious node pretending to be a different node in the network. The goal of the attack is to report false information about nodes one or two-hops away in order to maliciously affect the MPR selection process. Fig. 3.3(a) illustrates an example where node $x$ spoofs the identity of node $d$ and broadcasts Hello message advertising a valid link with node $c$. Then, node $a$ receives Hello messages from node $x$ indicating that node $d$ has links with nodes $c$ and 
$f$. In this case, node $a$ selects incorrectly node $d$ as the only element in its MPR set. In consequence, node $c$ is unreachable through the MPR set and never receives TC messages. Fig. 3.3(b), presents an example where the attacker affects the MPR selection of a node at distance two hops. The malicious node $x$ spoofs the identity of node $c$, i.e., nodes $f$ and $e$ generate Hello messages advertising node $c$ as a one-hop neighbor. From the point of view of node $a$, nodes $b, e, f$ and $d$ have node $c$ as a one-hop neighbor. As a result of the attack, node $a$ can select incorrectly nodes $f$ or $e$ as an MPR. In this case, nodes $b$ and $d$ do not forward control traffic information to node $c$ because they are not included in the MPR set.

Link Spoofing. The link spoofing attack [31] is performed by a malicious node that reports an inexistent link to other nodes in the network. The objective of the attacker is to manipulate the information about the nodes one or two hops away and be selected as part of the MPR set. Once the malicious node has been selected as an MPR, it neither generates nor forwards control traffic information. The flooding disruption attack due to link spoofing is illustrated in Fig. 3.4(a). In this example, node $x$ spoofs links to nodes $e$ and c. Node $x$ sends Hello messages and looks like the best option to be selected as an MPR for node $a$. Node $a$ receives the Hello messages from node $x$ and computes incorrectly its MPR set by selecting node $x$ as the only element to reach nodes $e$ and $c$. Thus, all routing information do not reach nodes two hops away from node $a$.

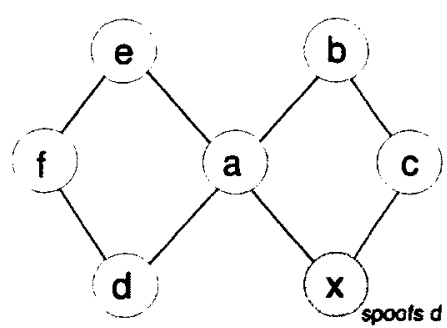

(a)

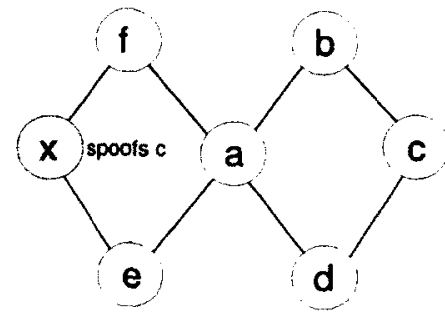

(b)

Figure 3.3: Flooding disruption due to identity spoofing attacks. In Fig. 3.3(a) node $x$ spoofs $d$ and reports an incorrect link between nodes $c$ and $d$ (one-hop address duplication). In Fig. 3.3(b), node $x$ spoofs $c$ and affects node $a$ 's MPR selection (two-hop address duplication). 
A variant of the attack can be performed by a misbehaving node either reporting a link to an inexistent node (i.e. a phantom node) or selecting an invalid MPR set. For instance, in Fig. 3.4(b), node $a$ is forced to select node $x$ as an MPR because is the only node to reach the inexistent node $w$. In the second case, a malicious node may disrupt the flooding of topology control information by misbehaving during the MPR selection process. Fig. 3.5(a) illustrates the attack. Node $x$ wants to be selected as the only MPR of node $a$. Then, it spoofs a link to node $g$ and generates Hello messages announcing node $g$ as a one-hop neighbor and its only MPR. From the perspective of node $a$, nodes $c$ and $g$ can be reached through node $x$. Then, node $x$ is the best candidate to be selected as an MPR for node $a$. Thus, node $x$ receives and forwards TC messages from node $a$. However, those messages never reach node $d$ because any one-hop neighbor of node $x$ retransmits the messages. This attack exploits the source dependent requirement in OLSR to forward control traffic information. In this case, for nodes $a, b, c$ and $e$, node $x$ is not included in their selector table and they never forward any message from node $x$.

\subsubsection{Incorrect Relaying}

A misbehaving node can disrupt the integrity of the network by either incorrectly generating or relaying control traffic information on behalf of other nodes. Consider $x$ in Fig. 3.5(a) as a misbehaving node. Node $x$ wants to be selected as the only MPR of node

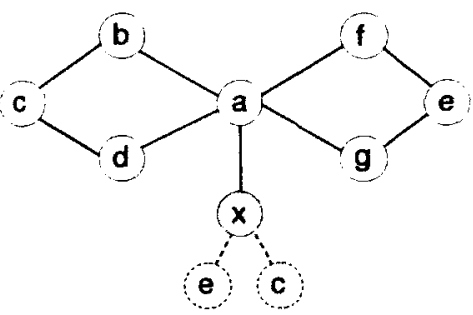

(a)

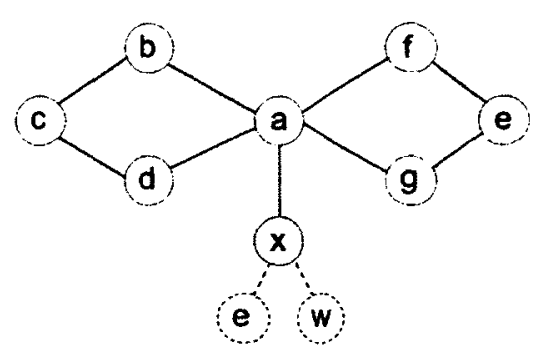

(b)

Figure 3.4: Flooding disruption due to link spoofing attacks. In Fig. 3.4(a), node $x$ spoofs links to nodes $e$ and $c$. In Fig. 3.4(b), node $x$ spoofs links to nodes $e$ and the inexistent node $w$. 
$a$. Then, it spoofs a link to node $g$ and generates Hello messages announcing node $g$ as a one-hop neighbor. From the perspective of node $a$, nodes $c$ and $g$ can be reached through node $x$. Thus, node $x$ is selected by node $a$ as its only MPR and might perform the following incorrect behaviors:

- Selfish behavior. The attack is performed by a node that misbehaves and neither generates nor forwards TC messages. To increase the effectiveness of the attack, the malicious node might establish false links to other nodes in the network and force its one-hop neighbors to select it as their MPR. Fig. 3.5(a) illustrates an example where node $x$ has been selected by node $a$ as an MPR but it does not relay control traffic on behalf of other nodes. As a result, node $d$ does not receive control traffic information from node $a$. Note that in OLSR, the attacker can choose not to forward any particular message, i.e., TC, MID or HNA messages.

- Slanderer behavior. Due to message size limitations, an MPR may report only a partial list of elements in its selector set, i.e., an MPR may generate more than one TC message to report its entire selector set. A receiver can not know if an MPR reports its entire selector set in more than one TC message. The information gathered from the TC messages is accumulated in its topology table and is only eliminated when the validity time has expired. Thus, a misbehaving node can always generate TC messages without reporting all nodes in its selector table claiming that the size

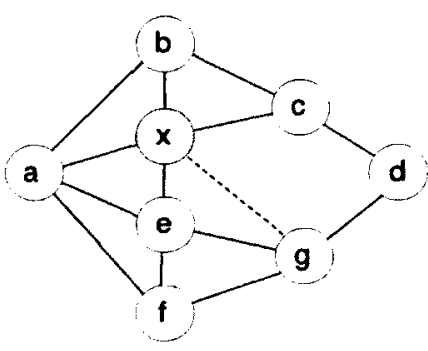

(a)

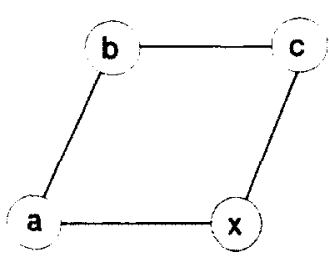

(b)

Figure 3.5: Flooding disruption due to protocol disobedience. In Fig. 3.5(a), node $x$ never selects a valid MPR set. In Fig. 3.5(b), node $x$ modifies and forwards incorrectly TC messages. 
of the messages is not enough to include all nodes in its selector table. As a result, if node $x$ generates TC messages without including node $a$, node $d$ is not able to compute a path to node $a$.

- Hop Limit attack. A malicious node $x$ may drastically decrease the hop limit (TTL value) when forwarding a TC message, e.g., setting the hop limit equal to zero. This reduces the scope of retransmitting the message. The attack can be performed by a malicious node that has not been selected as an MPR. For instance, in Fig. 3.5(b), a control message is forwarded by node $a$ and received by both nodes $x$ and $b$. Previously node $b$ was selected by node $a$ as its MPR. However node $x$ forwards the message without any delay or jitter such that its retransmission is received before the valid message from $b$ arrives. Before forwarding, it reduces the hop limit of the message. The affected node, node $c$, processes the message and mark it as already received, ignoring future valid copies from $b$. Thus, the message with a very low hop limit will not reach the whole network. 


\section{Chapter 4}

\section{Survey of the Literature}

\subsection{Introduction}

Vulnerabilities in OLSR have been studied extensively. For instance, in [9], Adjih et al. present security risks in the OLSR protocol and countermeasures based on cryptographic mechanisms to secure the protocol with or without compromised nodes in the network. The authors claim that an efficient securing mechanism should ensure the network integrity even when the network is subject to attacks that interrupt the connectivity. In $[32,31]$ Clausen and Herberg review security issues in OLSRv2. The authors analyze the basic algorithms that constitute the OLSRv2, and identify possible vulnerabilities and attacks.

Several authors have contributed with cryptographic mechanisms to secure OLSR. Cryptographic mechanisms are proposed to enforce: integrity, authentication and confidentiality. Thus, public-key encryption is used for confidentiality, digital signature for integrity of the messages and digital certificates for authentication. However, the implementation of a Public Key Infrastructure (PKI) in MANETs is difficult due to the lack of a central authority (CA). Additionally, the efficient distribution of public and private keys is a challenging problem. Timestamps are implemented with digital signatures to assure the freshness of the message. However, time synchronization is difficult to achieve particularly in MANETs.

According to Adjih et al. [9], a cryptographic capable node is a node that has received valid keys to sign and verify messages. A misbehaving node can be also a cryptographic capable node. In both cases, the connectivity of the network is compromised. Intrusion Detection Systems (IDS) [65, 21, 64] are implemented to analyze malicious behavior in the network. However, once a misbehaving node has been detected, an efficient reputation 
model is needed to convey to other nodes the results observed by the IDS. Although several efforts have been proposed to secure the OLSR protocol there are still security gaps that need to be addressed. In the following, we present some existing contributions to secure the OLSR protocol and other routing protocols based on OLSR. Existing countermeasures can be classified in: cryptographic mechanisms and IDS systems.

\subsubsection{Cryptographic Mechanisms}

In this section, we describe proposed solutions based on cryptographic mechanisms. In [33], Clausen et al. present a digital signature mechanism for authentication and authorization in OLSRv2. The authors introduce the concept of admittance control for OLSRv2 networks and suggest a security extension based on digital signatures. They compare several standard digital signature algorithms such as: RSA, DSA, ECDSA and HMAC. The goal is to enable trusted nodes and to disable non-trusted nodes from participating in the control message exchange between routers, thereby providing a mode-of-operation similar to traditional mechanism employed for preserving network integrity in routed networks. Additionally, a performance study of the propose extension is presented to quantify the impact of increased control traffic overhead and increased message generation as well as processing time. The authors observed that HMAC requires significantly less time than ECDSA, DSA and RSA for generating a message signature. For the verification of a message signature, HMAC likewise spends substantially less time than ECDSA and DSA, whereas RSA is close to HMAC. Verification of RSA signatures has much greater overhead but is faster than both ECDSA and DSA.

In [51], Joseph et al. present in detail routing attack scenarios and their elements. Also, authors introduce a security threat analysis that is used to explore different attack scenarios and behaviors in ad hoc routing protocols. Their objective is for understanding the security vulnerabilities and realizing the requisites of a security system design for ad hoc networks. Similarly, in [53] Kannhavong et al. present a survey of routing attacks in mobile ad hoc networks. 
In [83], Raffo et al., examined security issues related to the OLSR protocol, and enumerate a number of possible attacks against the integrity of the OLSR routing infrastructure. In particular, authors study attacks when a mechanism of digitally signed routing messages is deployed and an attacker may have taken control over trusted nodes. Their solution is based on inclusion of the geographical position of the sending node in control messages and on evaluation of reliability of links; this is accomplished using a GPS device and a directional antenna embedded in each node. Signatures with timestamps are sufficient to thwart attacks such as incorrect traffic generation and incorrect traffic relaying, when only legitimate nodes can sign control packets. Adding the node location in signature messages allows the network to avoid wormhole attacks and false messages generated by misbehaving nodes.

Raffo also presented in his Ph.D. thesis [84], a classification of possible attacks in OLSR networks. The author proposed a security architecture based on digital signatures. Additionally, the author proposed other techniques such as: reuse of previous topology information to validate the actual link state, cross-check of advertised routing control data with the node's geographical position, and intra-network misbehavior detection and elimination via flow coherence control or passive listening. Countermeasures in case of compromised nodes are also considered. Furthermore, the author assesses practical problems concerning the choice of a suitable symmetric or asymmetric cipher, alternatives for the algorithm of cryptographic key distribution, and the selection of a method for signature time stamping. In summary, the author presented an outline of different signature algorithms. The author suggested that the study and design of better cryptographic algorithms, i.e., algorithms that use a smaller signature size to reduce computation complexity would increase the suitability of his proposed OLSR security architectures.

In [41], Fourati and Agha propose a security algorithm fully distributed and autoorganized to secure OLSR networks based on the secret sharing idea. The algorithm is based on threshold cryptography mechanisms to ensure the integrity of routing messages. The proposed solution is optimized through the exploitation of the optimized MPR-based flooding in OLSR networks. Then, the augmentation of messages' number imposed by 
the sharing signature scheme does not affect significantly the routing specifications.

In [54], Khakpour et al., addressed the access control problem in MANETs. The authors proposed a hierarchical distributed AAA (Authentication, Authorization, and Accounting) architecture for proactive link state routing protocols. This proposal contains a lightweight and secure design of an overlay authentication and authorization paradigm for mobile nodes as well as a reliable accounting system to enable operators to charge nodes based on their connection time. The authors also suggest a hierarchical distributed AAA server architecture with a resource and location aware election mechanism. Moreover, this proposal mitigates the OLSR security issues and eventually defines a node priority-based quality of service. The design of the architecture targets a minimum signaling overhead as well as calculation cost. In fact, different tasks are fairly distributed among distributed AAA servers. The calculation cost and overhead signaling is trivial compared to OLSR signaling and routing computations.

In [85], Rana and Kapil propose an mechanism based on authentication checks of information injected into the network, mutual authentication between two nodes as well as other techniques such as authentic route confirmation ticket which regulates the behavior of nodes to prevent from internal attacks i.e. node misbehavior attack. Their approach allows only legitimate nodes to establish secure route rather than trying to detect adversary nodes after their involvement in the routing protocol.

\subsubsection{Intrusion Detection Systems}

In this section we describe proposed solutions based on Intrusion Detection Systems (IDS). IDS mechanisms such as: Watchdog/Pathrate [65], proposed by Marti et al., CONFIDANT (Cooperation Of Nodes: Fairness In Dynamic Ad hoc Networks) proposed by Buchegger and Le Boudec [21] or WATCHERS (Watching for Anomalies in Transit Conservation: a Heuristic for Ensuring Router Security) proposed by Bradley et al. [20, 46], are difficult to implement in MANETs. When the IDS detects a malicious behavior, it 
needs to notify other nodes in the network about the misbehaving node. Therefore some IDS protocols have been designed exclusively for MANETs.

In [6], Abdellaoui and Robert, proposed the SU-OLSR protocol (SU for suspicious) to prevent attacks against against OLSR-based routing protocols. In SU-OLSR the MPR selection is based on the trustworthiness of nodes. A malicious node might force its neighbors to choose it as an MPR node. Hence, a node should never select a neighbor as an MPR node if it behaves suspiciously and shows specific characteristics which would influence the MPR selection. Authors also show that to compute optimal paths, the optimality should not depend only on the length of a path but also whether or not it goes through fully or partially trusted MPR nodes. In [11], Adnane et al., proposed a trust based reasoning for OLSR that allows each node to correlate information provided by Hello, TC messages and data packets information so as to validate its local view of the global network topology. In their approach, when an inconsistency is detected between any received messages and its local view, the reasoning node is able to identify the compromised route. Their approach does not require any modification of the bare OLSR, but only the integration of the trust reasoning model on each node. Wu et al. present in [99] an overview of attacks according to the protocol layers, security attributes and mechanisms. Additionally, they present preventive approaches following the order of the layered protocol layers and an overview of reactive approaches based on IDS mechanism for MANET as a second line of defense to thwart attacks.

A similar idea is preseted by Adnane et al. to mitigate certain vulnerabilities in the OLSR protocol, authors propose in [12] the integration of a trust-based reasoning in every nodes. Thus, each node is able of identifying misbehaving nodes by analyzing received messages using the protocol trust rules. Although trust-based reasoning in OLSR nodes can also be useful for routing table validation, authors only focus on the MPR selection and propose that the MPR selection can be strengthened and validated by exploiting trust properties and relations.

Vilela et al., present in [94] a feedback reputation mechanism which assesses the integrity of routing control traffic by correlating local routing data with feedback messages 
sent by the receivers of control traffic. Based on this assessment, misbehaving nodes are shown to be reliably detected and can be adequately punished in terms of their ability to communicate through the network. In [38], Cuppens et al. investigate the use of AspectOriented Programming (AOP) in MANETs to provide availability issues in proactive routing protocols. Their approach is based on a detection-reaction process. Authors formally describe normal and incorrect node behaviors to derive security properties using AOP. The proposed algorithm verifies if those security properties are violated. If they are, then the detector node sends to its neighborhood the detection information to avoid choosing the intruder as part of valid paths to be constructed. A node chooses valid paths based on the reputation of other nodes.

In [52], Kannhavong et al. present a study of routing attacks in OLSR-based ad hoc networks. In particular, authors present a Node Isolation attack that results in a Denialof-Service (DoS) attack. To prevent the attack authors propose a countermeasure based on two phases: detection phase and avoidance phase. In the first phase, nodes analyze the retransmissions from their MPRs, once an attacker has been detected the affected node sends a modified Hello message with a request value. Thus, in the avoidance phase the receiving nodes, i.e. one-hop neighbors, will retransmit the $\mathrm{TC}$ messages from the node under attack even if that node is not part of their selector tables.

\subsection{OLSR extensions}

\subsubsection{Scalability}

Scalability is a challenge in MANETs. Hierarchical OLSR [96] has been proposed by Villasenor-Gonzalez to address routing in large and dense MANETs. HOLSR is explained in detail in Chapter 6. Additionally, Cluster OLSR(C-OLSR) [88] proposed by Ros et al., the Multi-level OLSR routing using the HNA extension (MORHE) proposed in [97] by Voorhaen et al., or the Tree Clustering $[17,16]$ mechanism proposed by Baccelli are based on OLSR to address security in MANETs. These other hierarchical approaches are also described in Chapter 6.

As an alternative, OLSR enhanced with Fish Eye routing techniques has been proposed 
to address scalability. In [8], Adjih et al., presented a study of how much the scalability in OLSR is enhanced with the use of Fish Eye techniques in addition to the link state routing framework. The authors compare OLSR and OSPF, both with Fish Eye enhancing techniques. Their results show that OLSR outperforms Fish Eye enhanced OSPF. In [74], Nguyen et al. propose an analytic model to study the scalability of the OLSR routing protocol. The authors focus on the protocol overhead and show that OLSR with the FishEye extension can support large and dense networks.

\subsubsection{Multicasting}

Multicast is the delivery of a message to a group of destination nodes simultaneously in a single transmission. The MPR flooding mechanism has been implemented to improve multicasting in routing protocols in MANETs. The Multicast Optimized Link State Routing protocol (MOLSR), is presented in [59] by Laouiti et al., MOLSR is designed to construct a multicast structure in order to route multicast traffic in MANETs. MOLSR works for mobile multicast routers and in heterogeneous networks composed of simple unicast OLSR routers. In [63, 62], Macker et al., present issues and performance analysis of optimized cover set algorithms supporting Simplified Multicast Forwarding(SMF). Authors, compare the classical flooding (CF) mechanism versus MPR-based algorithms such as: Source-specific Multipoint Relay (S-MPR), Non-source-specific MPR(NS-MPR), Essential Connected Dominating Set (E-CDS) and MPR-based CDS (MPR-CDS) algorithms.

In [72], Meraihi et al., evaluate and compare performances of three different multicast forwarding routing protocols in MANETs based on the selection of MPRs as a flooding mechanism. Authors compare the: Multicast Overlay Spanning Tree (MOST) [86] proposed by Rodolakis et al., Multicast OLSR (MOLSR) [59] proposed by Laouiti et al., and Simple Multicast OLSR (SMOLSR) $[2,68]$ presented by Minet et al., with respect to scalability and performance. 


\subsubsection{Multipath Routing}

Multipath routing has been proposed in MANETS to support application constraints such as reliability, load-balancing, energy-conservation and Quality-of-Service (QoS) [70]. Multipath OLSR(MP-OLSR) is proposed by Yi et al. in [103, 102, 104, 105]. MP-OLSR is explained in detail in Chapter 7.

In [55] Kun et al., propose a different version of multipath OLSR using IP-source routing. Based on Dijkstra's algorithm, the node calculate multiple disjoint paths. Additionally, authors introduce an algorithm of load-assigned to transmit data through the paths based on the congestion information of all intermediate nodes on each path. Badis and Al Agha [18], also propose a path selection criteria and QOLSR multi-path calculation based on bandwidth and delay. The resulting protocol computes multiple loop-free and node-disjoint paths. Authors implement the shortest-widest path algorithm to guarantee the loop-free property.

In [14], Aiache et al., propose an strategy to improve security and performance of an ad hoc network through a multipath routing strategy. Frequently, if security increases, the quality of service $(\mathrm{Q} o S)$ decreases. Authors, propose a solution that provides anonymity and security to Ad Hoc networks with a limited impact on QoS. Authors also give some security proofs of their solution for Ad Hoc networks.

In the following chapter we present our preventive mechanism to mitigate the effect of misbehaving nodes in OLSR-based networks. 


\section{Chapter 5}

\section{Mitigation of Flooding Disruption Attacks in OLSR}

\subsection{Introduction}

The Optimized Link State Routing (OLSR) [34] did not include security constrains in its original design. The core of the protocol is the selection, by every node, of Multipoint Relays (MPRs) among their one-hop symmetric neighbors. The nodes selected as MPRs are responsible of generating and forwarding control traffic messages and used to form optimal routes from a given node to any destination in the network. Thus, if an MPR fails or misbehaves sending or forwarding control traffic information, the connectivity of the network is compromised. The standard OLSR specification (RFC3626, Section 20), enumerates security considerations for confidentiality, integrity and interaction with external routing domains, but does not include security measures. Our research focus on mitigating control traffic attacks in OLSR.

Selecting the MPR sets as small as possible ensures that the overhead of the protocol is kept at minimum. However, adding extra coverage in the selection of the MPRs is an alternative solution to mitigate the effect of control traffic attacks. Additional MPR coverage, ensures that reachability for a node is advertised by more nodes. The RFC3626 [34], Section 16, defines an MPR coverage parameter (MPR_Coverage) to specify by how many MPRs any strict two-hop node should be covered. We present this approach in function $k$ covered-MPR. Nevertheless, the overhead of the protocol increases considerably due to excessive control traffic messages.

We propose function $k$-robust-MPR, in order to improve the selection of MPRs with additional coverage. Thus every node selects, when it is possible, $k+1$ disjoint MPRs sets. The union of the $k+1$ disjoint sets is a $k$-robust-MPR set, if we remove a maximum of $k$ elements from the MPR set of a given node $n$, all nodes two hops away from node 
$n$ are still covered by the remaining elements in the $k$-robust-MPR set. We tested functions $k$-robust-MPR and $k$-covered-MPR with the presence of two types of misbehaving nodes. One adversary interrupts the proper flooding of the control traffic messages, and the second one, generates them incorrectly. Our results show that our improved MPR selection offers a better balance between the protection against control traffic attacks and the overhead generated by the increased number of messages.

\subsubsection{Control Traffic Attacks to OLSR}

The generation and exchange of critical information are important vulnerability targets. The control messages flood the network to allow every node to create optimal paths to any destination in the network. If a node misbehaves by generating or forwarding incorrect control traffic information the integrity of the network is compromised. During the execution of the protocol, each node broadcasts Hello messages to advertise their presence among their one-hop neighbors, to learn about their two-hop neighbors and to select its MPRs. The MPRs generate and retransmit Topology Control(TC) Messages. The information from Hello and TC messages allows every node to construct their routing tables. Thus, in an OLSR network, the nodes have two principal tasks to perform [94]: (1) to generate correctly routing information (i.e., Hello and TC messages), and (2) to correctly relay traffic on behalf of other nodes in the network.

A target of an attack can be that legitimate nodes never receive correct control traffic messages or store incorrect information to affect negatively the network topology. The attacks at the routing level can be classified in two categories [38]: incorrect traffic generation and incorrect traffic relaying.

- Incorrect traffic generation: a node misbehaves by generating incorrect Hello or TC messages under a false identity or generating control traffic messages reporting an incorrect set of links. An attacker can either hide valid links or insert non-existing links. In all cases, the network connectivity is disrupted.

- Incorrect traffic relaying: if a node decides to drop valid packets, e.g., an MPR 
refuses to forward TC messages, the network will experience a degradation of communication. Equally, an attacker misbehaves by resending old valid control messages (not timestamped), or forwarding altered control messages. The nodes in the network will receive wrong information and update their routing tables with stale information.

Additionally, two or more nodes can collude to perform an attack. For instance, misbehaving nodes can establish invalid links and replay valid information in different regions of an OLSR network mounting a wormhole attack. This type of attack is particularly severe, because it is difficult to detect even in a network where security constraints (i.e., authentication, integrity and confidentiality) are implemented.

\subsubsection{Contributions of the Chapter}

In this chapter, we propose an improved MPR selection with additional coverage (cf., function $k$-robust-MPR) [23] to mitigate the effect of traffic control message attacks. We compare our function against the MPR selection with additional coverage (cf., function $k$ covered-MPR) proposed in the RFC3626. We analyze the cost and benefit of functions $k$ covered-MPR and $k$-robust-MPR for the selection of MPR with extra coverage in the presence of misbehaving nodes in an OLSR network. We also present the correctness of both functions in Section 5.3.3. Our function $k$-robust-MPR, mitigates the effect of the adversaries performing control traffic attacks and affecting negatively the construction of the routing tables for every node in an OLSR network. We also compare functions $k$ covered-MPR and $k$-robust-MPR to measure the level of mitigation against two types of adversaries. The experiments show that it is possible to offer equivalent protection but reducing the overhead due to the excessive number of control traffic messages generated by function $k$-covered-MPR. Additionally, function $k$-robust-MPR increases the performance ratio between the number of nodes with complete routing tables and the increased number of TC messages.

\subsubsection{Related Work}

The MPR selection process appears at the earliest stage of the OLSR routing protocol. The standard procedure used in the standard OLSR protocol for the selection of MPRs 
is a mix of greedy and most restrictive rule first strategies. Minimization of the number of nodes in the resulting MPR set has been addressed, for example, in [37, 73, 22]. The objective of the OLSR protocol is to optimize the flooding of topological information. According to this improvement, a node is included in the topology forwarding CDS iff (1) its identifier is the smallest in the neighborhood; or (2) it was selected as an MPR by the node with smallest identifier in the neighborhood. Notice that this method requires a numeric labeling of nodes. The identifier can be based, for instance, on the IP address of the nodes, or any other unique ordering of nodes. A variation of the previous proposal was presented in $[100,101]$ where the inclusion to the forwarding CDS satisfies the following two conditions: (1) the node has the smallest identifier in the neighborhood and, at least, two unconnected neighbors; or (2) it was selected as an MPR by the node with smallest identifier in the neighborhood.

Our scheme focuses on service availability and security by implementing an improved MPR selection with additional coverage. In the literature, the MPR_Coverage parameter is implemented to enhance fault tolerance, load balancing, reliability and Quality-ofService(QoS) [70, 103]. However, they do not analyze their approaches with a security perspective. An improvement of the OLSR flooding method presented in [10] proposes the transformation of MPRs into Connected Dominating Sets (CDS). The use of these CDS-based algorithms for the flooding of topological data as a replacement of the standard flooding procedure of OLSR is claimed by the authors as a promising trade-off between minimization and reliability. However, they do not address the use of an additional coverage in the selection of the MPR sets, for tuning the flooding process once the CDS has been built.

In [95] authors provide a trade-off between selecting MPRs sets with additional coverage using the MPR_Coverage parameter proposed in [34] and redundancy in the link-state information (TC_Redundancy parameter) to improve the QoS of the protocol. Nonetheless, they analyze their approach using the MPR with extra coverage proposed in [34] and without the presence of misbehaving nodes. In [93], authors prove that an MPR selection with additional coverage can be used to preserve $k$-connectivity in an OLSR network; based on 


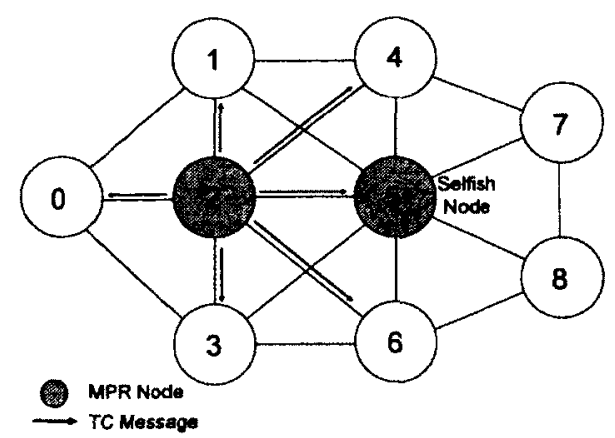

(a) Selfish Attack.

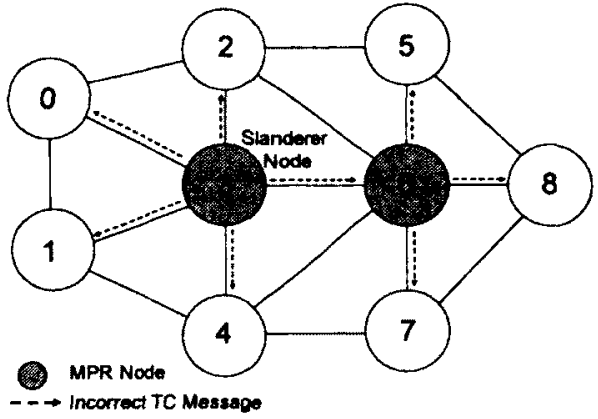

(b) Slanderer Attack

Figure 5.1: Example of a selfish and a slanderer attack. In (a), node 5 is a selfish node and has been selected by node 2 as an MPR. In (b), node 3 is a slanderer node and has been selected by node 1 as an MPR.

that approach we improve the selection of MPRs with additional coverage to reduce the overhead generated for excessive control messages and to mitigate traffic control attacks.

\subsection{Adversary Model}

In this section, we describe the characteristics of our adversaries. Flooding the network with TC messages allows nodes to construct a path to all destinations. The TC messages are generated exclusively by the MPRs, each MPR includes in the messages all its selector nodes. Thus, the receptor of the message learns that the original sender is the last-hop to all the nodes included in the message. If an MPR misbehaves generating or forwarding TC messages, then the connectivity of the entire network is compromised. In [38], authors define node profiles according to their behavior in a network during the execution of the OLSR protocol. We select the Selfish and Slanderer node profiles as active attackers.

- A Selfish node, is an MPR that decides neither to generate nor to retransmit TC messages. Figure 5.1(a) is an example of a selfish attack. For instance, consider node 2 as an MPR of node 0 , and node 5 an MPR of node 2 . If node 2 broadcasts a TC message, then node 5 might be responsible to retransmit the message but may decide not to do so. In consequence, nodes 7 and 8 will never learn that the last-hop to reach node 0 is node 2 . 
- A Slanderer node, is an MPR that generates incorrect information, i.e., an MPR that does not declare a complete MPR Selector Set. Figure 5.1(b), is an example of a slanderer attack. Node 3 has been selected by nodes 0,1,2 and 4 as an MPR. Node 3 is an attacker and generates a TC message but without including node 1 in the message. Node 6 receives and forwards the message. In consequence, node 8 will never be aware of the presence of node 1 .

These misbehaving nodes affect the integrity and the proper construction of routing tables for each node in the network. The nodes can be isolated and will not compute a complete view of the network topology. We consider that a node's routing table is affected if at least one element for a valid path in the network is missing. In both cases, alternative paths between nodes can help to mitigate the attacks. For instance, in Figure 5.1(a) node 2 can select node 5 or nodes 4 and 6 as MPRs. Thus, node 2 can reach nodes 7 and 8 trough nodes 4 and 6 . We analyze in the sequel two approaches for additional coverage in the selection of MPRs: the function presented in the RFC3626 [34] (cf., function $k$-coveredMPR), and an alternative selection of disjoint MPR sets (cf., function $k$-robust-MPR). The objectives are to minimize the overhead generated by an increased number of TC messages in the network and to offer equivalent protection in the presence of misbehaving nodes.

\subsection{MPR Computation with Additional Coverage}

This section describes the selection of MPRs with additional coverage presented in the RFC3626 [34] and our proposed function $k$-robust-MPR choosing disjoint groups of MPRs. In [34], additional coverage is defined as the ability of a node to select redundant MPRs. The selection of MPRs must be as small as possible to reduce the overhead due to flooding the network with TC messages. Nevertheless, additional coverage allows a node to advertise its presence to more nodes in the network. In this manner, extra coverage helps to maintain the integrity of the network in spite of the presence of misbehaving nodes during the execution of the OLSR protocol. However, the overhead generated by the increased number of TC messages reduces the performance of the network. This problem is addressed with our improved function $k$-robust-MPR (cf. subsection 5.3.2), which balances additional coverage and traffic overhead. 


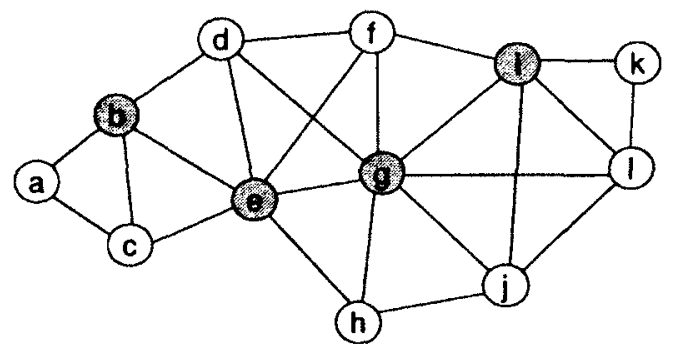

(a) MPR selection without additional coverage.

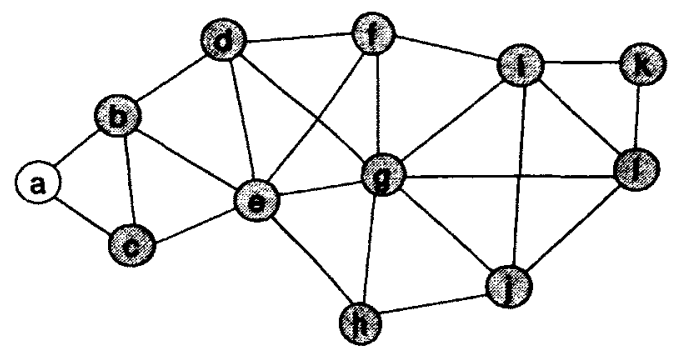

(b) $k$-Covered-MPR selection $k$ equal to two.

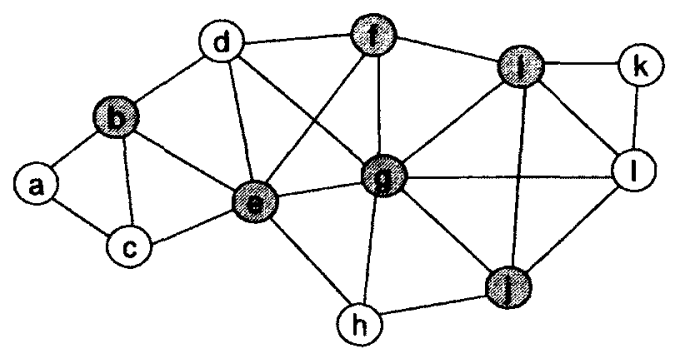

(c) $k$-Robust-MPR selection $k$ equal to one.

Figure 5.2: MPR selection in an OLSR network with and without additional coverage. Consider gray nodes as MPRs.

\subsubsection{RFC3626's MPR Coverage Parameter}

The RFC3626 [34] defines the MPR_Coverage parameter to specify by how many onehop nodes any two-hop neighbors must be covered. If MPR_Coverage is equal to one then the overhead is kept at minimum and the function is equivalent to the MPR selection without additional coverage specified in [34], Section 8.3.1. If MPR_Coverage is equal to $k$, a node selects its MPR set such as any two-hop neighbor is covered by $k$ one-hop neighbors, whenever possible. A poorly covered node, is a node in the two-hop neighborhood that cannot be covered by at least $k$ nodes in the one-hop neighborhood. The MPR_Coverage parameter is local to every node in the network. Nodes with different values of MPR_Coverage may operate in a same network. Function $k$-covered-MPR describes the MPR selection with coverage $k$ defined in [34], Section 16.1. To explain functions $k$-covered-MPR and $k$-robust-MPR, we will use the following notation:

- $d(n, u)$ : number of hops between nodes $n$ and $u$.

- $N_{1}(n):=\left\{n_{1}: d\left(n, n_{1}\right) \leq 1\right\}$.

- $N_{\leq 2}(n):=\left\{n_{2}: d\left(n, n_{2}\right) \leq 2\right\}$. 
- $N_{2}(n):=N_{\leq 2}(n) \backslash N_{1}(n)$.

- degree $\left(n, n_{1}\right):$ returns the number of nodes in $N_{2}(n)$ such that $N_{1}\left(n_{1}\right) \cap N_{2}(n) \neq \emptyset$, assuming that $n_{1} \in N_{1}(n)$.

- $M: M$ is an MPR set for node $n \Leftrightarrow M \subseteq N_{1}(n)$ such that for every node $n_{2} \in N_{2}(n), N_{1}\left(n_{2}\right) \cap M \neq \emptyset$.

- reachability $\left(n, n_{1}, A\right)$ : returns the number of nodes in $N_{2}(n)$ such that $d\left(n_{1}, n_{2}\right) \leq 1$ and $N_{1}\left(n_{2}\right) \cap A=\emptyset$, assuming that $A \subseteq N_{1}(n)$ and $n_{1} \in N_{1}(n) \backslash A$.

- required $(n, A):$ returns a set $B$, such that $B \subseteq N_{1}(n)$, and for every $b \in B$, $d(b, a) \leq 1$, assuming that $a \in A$ and $A \subseteq N_{2}(n)$.

- linked $(n, A)$ : returns a set $B$, such that $B \subseteq N_{2}(n)$, and for every node $b \in B$, $N_{1}(b) \cap A \neq \emptyset$, assuming that $A \subseteq N_{1}(n)$.

- nextNode $(n, A, B)$ : returns a node $a \in A$ such that $a$ provides the largest degree $(n, a)$ and reachability $(n, a, B)$. We assume that $A, B \subseteq N_{1}(n)$ and $A \cap B=\emptyset$.

- PoorlyCovered: subset of $N_{2}(n)$ formed by every node $n_{2} \in N_{2}(n)$ such that $\left|N_{1}\left(n_{2}\right) \cap N_{1}(n)\right|<k$.

- WellCovered: subset of $N_{2}(n)$ formed by every node $n_{2} \in N_{2}(n)$ such that $\left|N_{1}\left(n_{2}\right) \cap N_{1}(n)\right| \geq k$.

Function $k$-covered-MPR, with respect to a given node $n$ works as follows [34]:

1. First, we obtain the poorly covered nodes in $N_{2}(n)$. Then, we include in the MPR set $M$, the nodes in $N_{1}(n)$ that poorly cover nodes in $N_{2}(n)$.

2. We remove the poorly covered nodes from $N_{2}(n)$.

3. While there exist nodes in $N_{2}(n)$ not yet covered by at least $k$ nodes in the MPR set:

- We add to $M$ the node $n_{1}$ in $N_{1}(n)$ not in the MPR set, that provides the largest reachability $\left(n, n_{1}, M\right)$ and degree $\left(n, n_{1}\right)$. 
- We eliminate all the nodes in $N_{2}(n)$ now covered by at least, $k$ nodes in the MPR set.

For instance, according to the example presented in Figure 5.2, the MPR sets selected by all the nodes, derived in polynomial time by function $k$-covered-MPR, corresponds to column four in Table 5.1. Due to the additional MPRs selection, the number of TC messages increases considerably the amount of traffic in the network. In the sequel, we describe an improved function to select MPR sets with extra coverage that presents a balanced trade-off between additional coverage and traffic overhead.
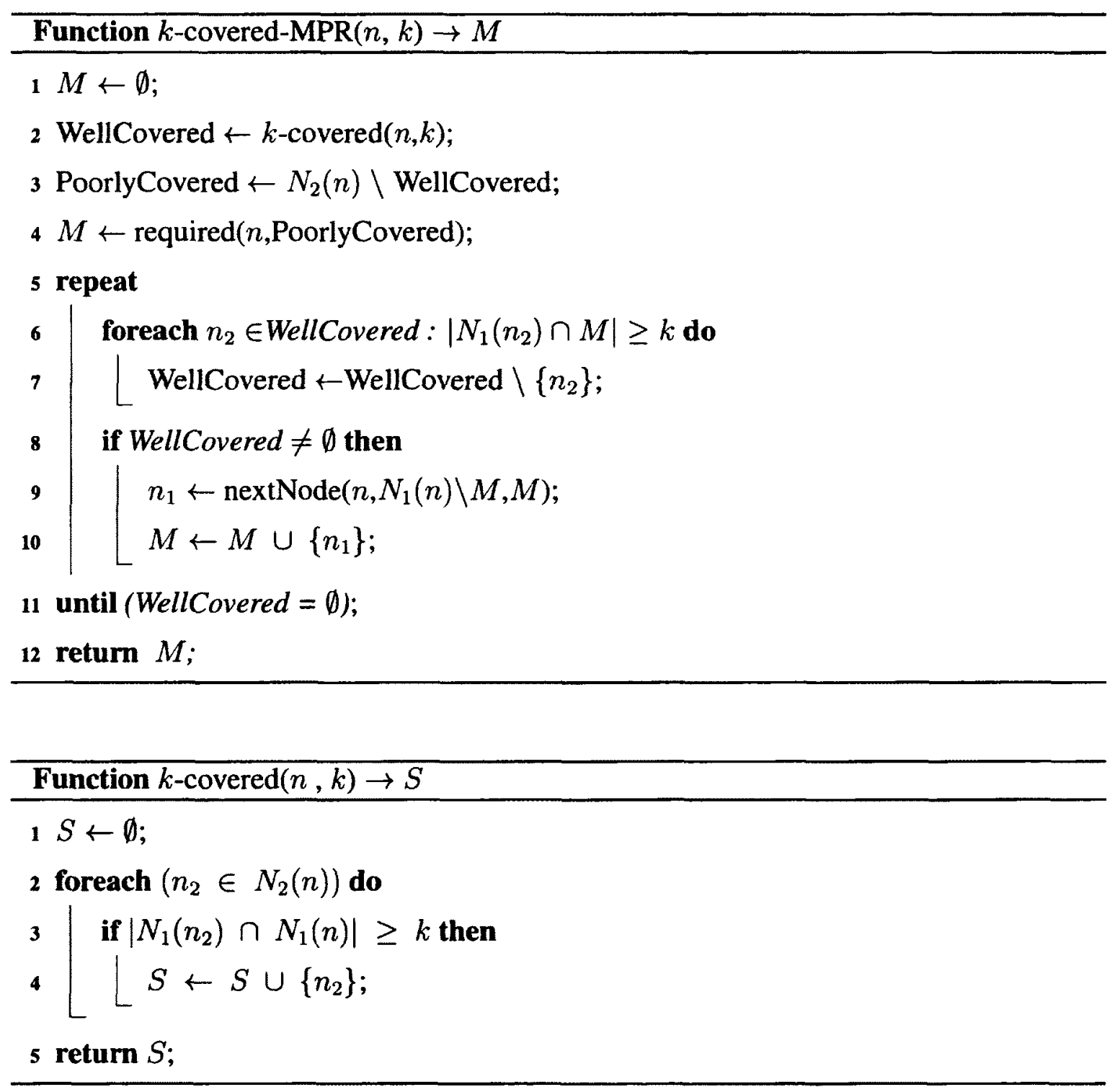

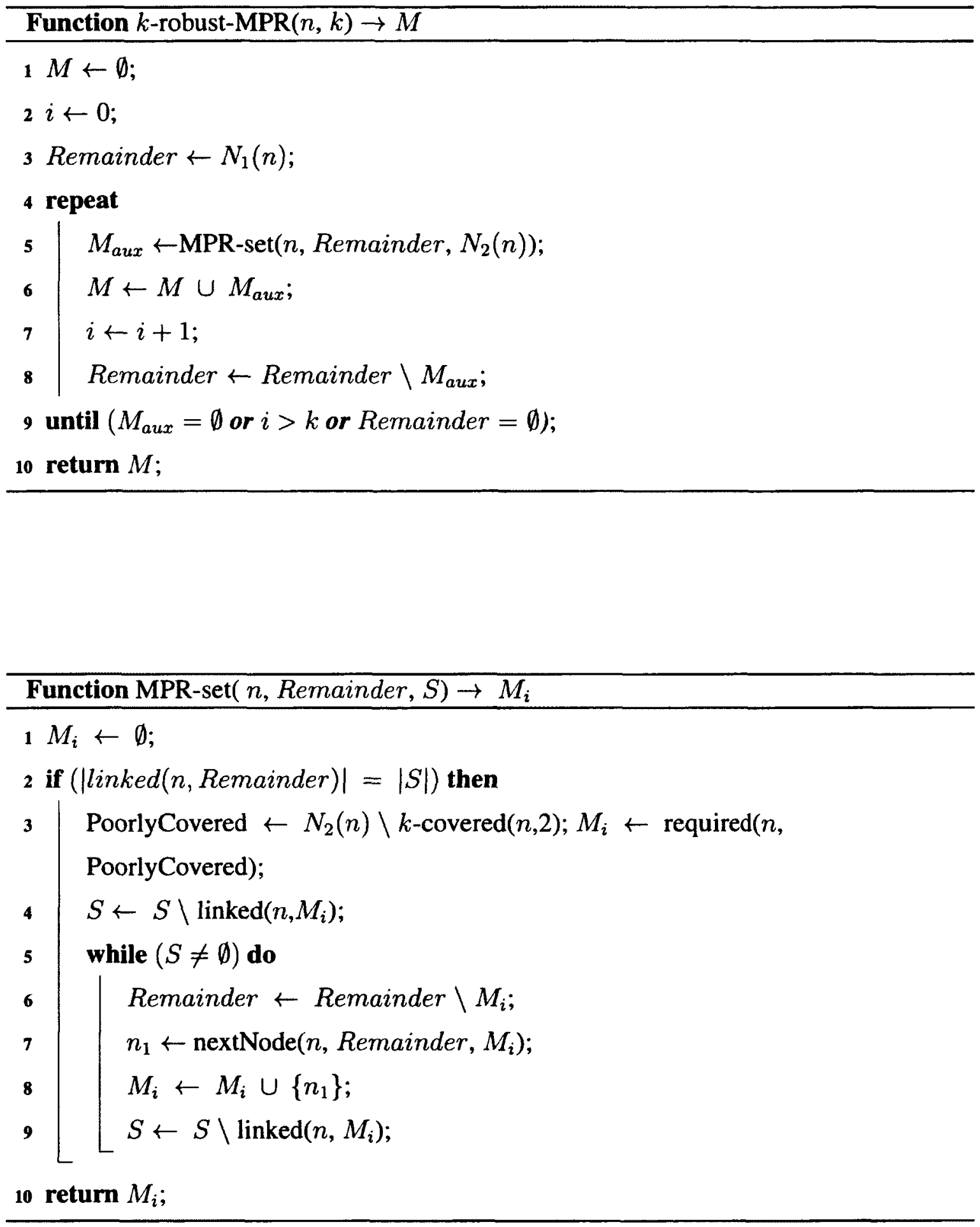


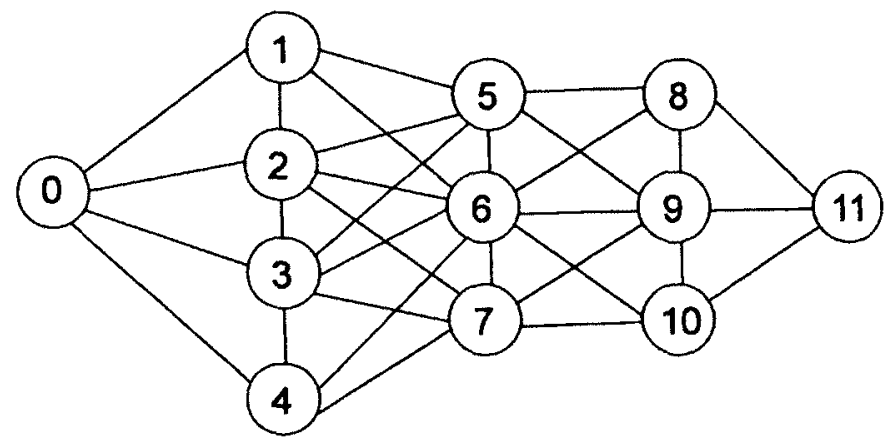

Figure 5.3: Consider nodes 2, 3, 6 and 9 as MPRs, selected with no extra coverage.

\subsection{2 $k$-Robust-MPR Selection}

In this section, we describe function $k$-robust-MPR that improves the selection of MPR sets with additional coverage. Function $k$-robust-MPR computes an MPR set that is composed of, at most, $k+1$ disjoint groups, i.e., every two-hop node is covered, if possible, by $k+1$ disjoint groups of one-hop neighbors. Function $k$-robust-MPR works as follows:

1. First, by invoking function MPR-set, we obtain a subset $M_{i}$ such that $M_{i}$ is subset of $N_{1}(n)$ and covers all the nodes in $N_{2}(n)$.

2. We repeat the function until it is not possible to find a new disjoint subset $M_{i}$ that covers all the nodes in $N_{2}(n)$ or we have found a maximum of $k+1$ disjoint subsets.

3. The MPR set is formed by the union, if it is possible, of $k$ disjoint subsets $M_{i}$.

If we apply function $k$-robust-MPR to the example depicted by Figure 5.3, then we obtain the set of MPRs shown in Table 5.1, column five. For example, the execution of function $k$ robust-MPR on node 0 , with parameter $k$ equal to two, returns the MPR set $\{2,3,1,4\}$, which is 2 -robust.

\subsubsection{Correctness of the Functions}

In this section, we demonstrate the correctness of functions $k$-covered-MPR and $k$-robustMPR. 
Lemma 1 Let $S$ be the set returned by applying function $k$-covered to node $n$. Every node in $N_{2}(n)$ covered by at least $k$ nodes in $N_{1}(n)$, is in $S$.

Proof Suppose that if we apply the function $k$-covered to node $n$ then it is possible to have a node $n_{2}$ in $N_{2}(n)$ such that $\left|N_{1}\left(n_{2}\right) \cap N_{1}(n)\right|$ is greater than or equal to $k$ and $n_{2}$ is not in $S$. However, according to the definition of function $k$-covered, every node $n_{2}$ in $N_{2}(n)$ is inspected by the foreach-loop and, if $\left|N_{1}\left(n_{2}\right) \cap N_{1}(n)\right|$ is greater or equal to $k$, we add node $n_{2}$ to $S$ in the body of the loop. Thus, $S$ is equal to the union of every node in $N_{2}(n)$ covered by at least $k$ nodes in $N_{1}(n)$.

Theorem 1 Let $M$ be the set obtained by applying function $k$-covered-MPR to node $n$. Every node in $N_{2}(n)$ with exactly $k^{\prime}$ neighbors in $N_{1}(n)$, such that $k^{\prime}$ is less than $k$, is covered by exactly $k^{\prime}$ nodes in $M$.

Proof Consider $M$ as the set obtained by applying function $k$-covered-MPR to node $n$. Then, suppose that there exists a node $n_{2}$ in $N_{2}(n)$ with exactly $k^{\prime}$ neighbors in $N_{1}(n)$, $k^{\prime}$ less than $k$, that is not covered by $k^{\prime}$ nodes in $M$. However, by applying function $k$ covered-MPR, in line 2, and according to Lemma 1, we assign to the set WellCovered every node in $N_{2}(n)$ covered by at least $k$ nodes in $N_{1}(n)$. In line 3 , we assign to the PoorlyCovered set all the nodes in $N_{2}(n)$ not in the set WellCovered. Then in line 4, for every node $n_{2}$ in PoorlyCovered, we assign to $M$ every node $n_{1}$ in $N_{1}(n)$ such that $N_{1}(n) \cap N_{1}\left(n_{2}\right)$ is not equal to the empty set. Therefore, every node in $N_{2}(n)$ with $k^{\prime}$ neighbors in $N_{1}(n)$ is covered by exactly $k^{\prime}$ nodes in $M$.

\begin{tabular}{|c|c|c|c|c|}
\hline Node & $\begin{array}{c}\text { Possible disjoint } \\
\text { MPR sets }\end{array}$ & $\begin{array}{c}\text { No extra } \\
\text { coverage }\end{array}$ & $\begin{array}{c}k \text {-covered-MPR } \\
k=2\end{array}$ & $\begin{array}{c}k \text {-robust-MPR } \\
k=2\end{array}$ \\
\hline 0 & $\{\{2\},\{3\},\{1,4\}\}$ & $\{2\}$ & $\{2,3\}$ & $\{2,3,1,4\}$ \\
\hline 1 & $\{\{6\}\}$ & $\{6\}$ & $\{6,5,2,0\}$ & $\{6\}$ \\
\hline 2 & $\{\{6\},\{7,5\}\}$ & $\{6\}$ & $\{6,7,5\}$ & $\{6,7,5\}$ \\
\hline 3 & $\{\{6\},\{5,7\}\}$ & $\{6\}$ & $\{6,5,7\}$ & $\{6,7,5\}$ \\
\hline 4 & $\{\mid 6\}\}$ & $\{6\}$ & $\{6,7,3,0\}$ & $\{6\}$ \\
\hline 5 & $\{\{3,9\},\{6,2,8\}\}$ & $\{3,9\}$ & $\{6,3,9,2,8\}$ & $\{3,9,6,2,8\}$ \\
\hline 6 & $\{\{2,9\},\{3,8\},\{1,10\}\}$ & $\{2,9\}$ & $\{2,3,9,8\}$ & $\{2,3,9,8\}$ \\
\hline 7 & $\{\{2,9\},\{6,3,10\}\}$ & $\{2,9\}$ & $\{6,2,9,3,10\}$ & $\{2,9,6,3,10\}$ \\
\hline 8 & $\{\{6\}\}$ & $\{6\}$ & $\{6,5,9\}$ & $\{6\}$ \\
\hline 9 & $\{\{6\}\}$ & $\{6\}$ & $\{6,5,7\}$ & $\{6\}$ \\
\hline 10 & $\{\{6\}\}$ & $\{6\}$ & $\{6,7,9\}$ & $\{6\}$ \\
\hline 11 & $\{\{9\}\}$ & $\{9\}$ & $\{9,8,10\}$ & $\{9\}$ \\
\hline
\end{tabular}

Table 5.1: MPR computation examples in figure 5.3. 
Theorem 2 Let $M$ be the set obtained by applying function $k$-covered-MPR to node $n$. Then, every node in $N_{2}(n)$ with exactly $k^{\prime}$ neighbors in $N_{1}(n)$, such that $k^{\prime}$ is greater than or equal to $k$, is covered by, at least, $k$ nodes in $M$.

Proof Consider $M$ as the set with coverage $k$ obtained by applying function $k$-coveredMPR to node $n$. Then, suppose that there exists a node $n_{2}$ in $N_{2}(n)$ with exactly $k^{\prime}$ neighbors in $N_{1}(n), k^{\prime}$ greater than or equal to $k$, that is not covered by at least $k$ nodes in $M$. However, in line 2, we assign to the set WellCovered all nodes in $N_{2}(n)$ covered by at least $k$ nodes in $N_{1}(n)$. We assign to the PoorlyCovered set every node in $N_{2}(n)$ not in WellCovered. Thus, according to Theorem 1, we assign to $M$ the nodes in $N_{1}(n)$ that poorly cover all nodes in the PoorlyCovered set. Then, we repeat the following procedure until every node in WellCovered is covered by, at least, $k$ nodes in $M$ :

- In lines 6 and 7, we eliminate every node in the set WellCovered covered by, at least, $k$ nodes in $M$.

- If WellCovered is not equal to the empty set, then we add to $M$ the node in $N_{1}(n)$ that covers the largest number of elements in $N_{2}(n)$.

Therefore, if $k^{\prime}$ is greater than or equal to $k$, every node in $N_{2}(n)$ with $k^{\prime}$ neighbors in $N_{1}(n)$, is covered by, at least, $k$ nodes in $M$.

Lemma 2 Let $M_{i}$ be a set obtained by applying function MPR-set to node n, such that $M_{i}$ is not equal to the empty set and $M_{i} \subseteq N_{1}(n)$. Then $M_{i}$ successfully covers all nodes in $N_{2}(n)$ (i.e., $M_{i}$ is a valid MPR candidate).

Proof Consider that after applying function MPR-set to node $n$ with parameters $\mathrm{Re}$ mainder, a subset of $N_{1}(n)$, and $S$, equal to $N_{2}(n)$, it is possible to obtain a set $M_{i}$ not equal to the empty set, such that $M_{i}$ does not cover all elements in $N_{2}(n)$. However, by applying function MPR-set to node $n$, if $\mid \operatorname{linked}(n$, Remainder $) \mid$ is equal to $|S|$, then we can construct a set $M_{i}$, such that $M_{i}$ covers all elements in $S$. In function MPR-set line 3, we add to $M$ every node in Remainder that is the only one to provide reachability to a node in $S$. After that, while there exist nodes in $S$ that are not yet covered by, at least, one node in $M_{i}$, we select a new node $n_{1}$ from Remainder not in $M_{i}$ that covers the largest number of nodes in $S$. Thus, we obtain $M_{i}$ such that $\left|\operatorname{linked}\left(n, M_{i}\right)\right|$ is equal to $|S|$. Therefore, if 
$M_{i}$ is not equal to the empty set, we can affirm that every node in $S$ is covered by, at least, one node in $M_{i}$ and so that every set $M_{i}$ returned by function $M P R$-set is, indeed, a valid MPR set.

Theorem 3 Let $M$ be a set obtained by applying function $k$-robust-MPR to node $n$. Then $M$ is a valid MPR set, i.e., $M$ covers all the nodes in $N_{2}(n)$.

Proof Suppose that by applying function $k$-robust-MPR we obtain a set $M$, such that that $M$ is not a valid MPR set. However, according to Lemma 2, if $M_{i}$ is not equal to the empty set, then $M_{i}$ is a valid MPR set. Notice that, function $k$-robust-MPR invokes function MPR-set $k^{\prime}+1$ times, such that $k^{\prime}$ is greater than or equal to zero and less than or equal to $k$. Since $M$ is the union of $k^{\prime}+1$ disjoint valid MPR sets, we can affirm that $M$ is a valid MPR set.

Corollary 1 Let $M$ be a valid MPR set obtained by applying function k-robust-MPR to node $n$. If a node in $N_{2}(n)$ is covered by $k^{\prime}$ nodes in $N_{1}(n)$, with $k^{\prime}$ less than or equal to $k$ and there is no $k^{\prime \prime}$ in $N_{1}(n)$ such that $k^{\prime \prime}$ is less than $k^{\prime}$. Then, it is possible to invoke a maximum of $k^{\prime}$ times function MPR-set and $M$ is a $k^{\prime}$-robust-MPR set.

Proof Suppose that there exists a node $n_{2}$ in $N_{2}(n)$ such that $n_{2}$ is covered by $k^{\prime}$ nodes in $N_{1}(n)$, then it is possible to invoke function MPR-set $k$ times to obtain $k$-robust-MPR set. However, by applying function $k$-robust-MPR to node $n$, we invoke function MPR-set with parameters $n$, Remainder and $S$. Initially, in line 3 we assign to Remainder the set $N_{1}(n)$, and every time a valid $M_{i}$ is obtained from function MPR-set, we subtract the set $M_{i}$ from Remainder. Thus, if a node in $N_{2}(n)$ is covered by $k^{\prime}$ nodes in $N_{1}(n)$, after invoking function MPR-set $k^{\prime}$ times, then there exists a node $n_{2}$ in $N_{2}(n)$ that is not covered in Remainder and $\mid$ linked(n, Remainder $) \mid$ is less than $\left|N_{2}(n)\right|$. Then, function MPR-set returns the empty set and $M$ is equal to the union of $k^{\prime}$ valid disjoint $M_{i}$ sets.

Theorem 4 Let $M$ be the set obtained by iteratively applying $k^{\prime}+1$ times function MPRset from function $k$-robust-MPR with parameters $n$ and $k$, such that $k^{\prime}$ is greater than zero and less than or equal to $k$. Then, for any $S$ subset of $M$ of size $k^{\prime}$, the nodes in $M$ not in $S$ still cover all the nodes in $N_{2}(n)$. 
Proof Suppose that $M$ is a valid MPR set obtained by applying function $k$-robust-MPR to node $n$, and the elements in $M$ not in $S$ do not cover all nodes in $N_{2}(n)$. However, every $M_{i}$ in $M$ is obtained by applying $k^{\prime}$ times function MPR-set to node $n$. According to Corollary 1 , we have $k^{\prime}+1$ valid $M_{i}$ sets in $M$. We consider the worst case when every element in $S$ belongs to a different $M_{i}$ and we have $k^{\prime}$ invalid $M_{i}^{\prime}$ sets in $M$. Consider $M^{\prime}$ equal to the union of $k^{\prime}$ invalid $M_{i}^{\prime}$, i.e., $M$ is equal to the union of $M_{k^{\prime}+1}$ and $M^{\prime}$ such that, according to the definition of function MPR-set, $\left|\operatorname{linked}\left(n, M_{k^{\prime}+1}\right)\right|$ is equal to $\left|N_{2}(n)\right|$. Then, $M_{k^{\prime}+1}$ is a valid MPR set and the elements in $M$ not in $M^{\prime}$ still cover all nodes in $N_{2}(n)$. Therefore, nodes in $M$ not in $S$ are a valid MPR set.

In the next section, we compare functions $k$-covered-MPR and $k$-robust-MPR in the presence of selfish and slanderer nodes to evaluate the benefits of both functions.

\subsection{Experiments}

We conducted simulations to confirm that our $k$-robust-MPR set selection allows to minimize the effect of misbehaving nodes and helps to reduce the overhead generated by the $k$-covered-MPR function proposed in the standard OLSR protocol. To measure the effectiveness of our proposal, we count the number of nodes that were able to find a path to all nodes in the network after executing the two different MPR selection methods, for a certain period of time. Additionally, we take into account, the number of retransmissions during the simulations. We conducted our simulations using the NS-2 simulator [66], version 2.29, with the UM-OLSR [87] package. We modified the original UM-OLSR code to implement the $k$-robust and $k$-covered MPR functions.

The RFC3626 considers that nodes with different characteristics can coexist in an OLSR network. For our experiments, we assume that all the nodes have the same characteristics, every node has just one interface and all the links between the nodes are bidirectional. Additionally, all the nodes have the same willingness to carry and forward traffic on behalf of other nodes, except for those that have been selected as misbehaving nodes. The misbehaving nodes do not collude to perform an attack. Table 5.2 describes the parameters for our simulations in NS-2. In our experiments, no data traffic is generated and all the scenarios are static. 


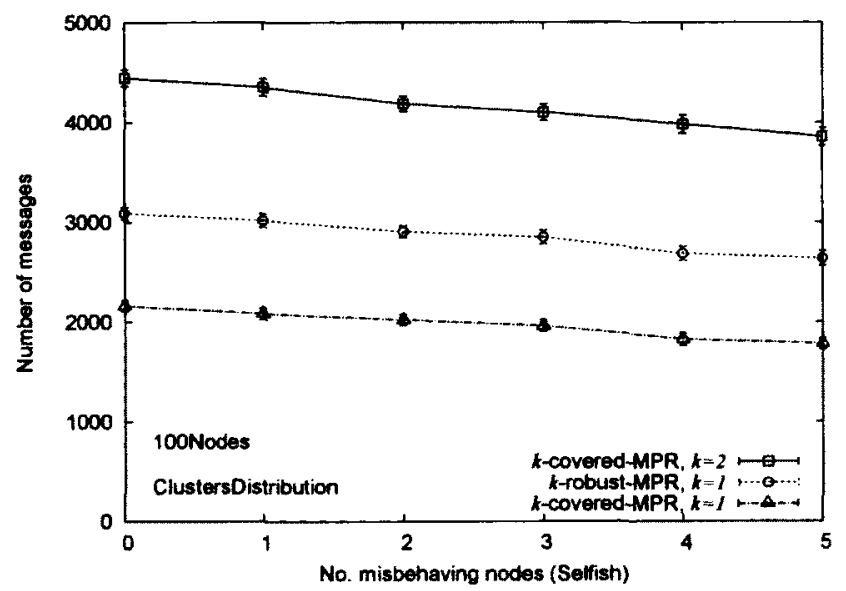

(a) Number of Topology Control messages.

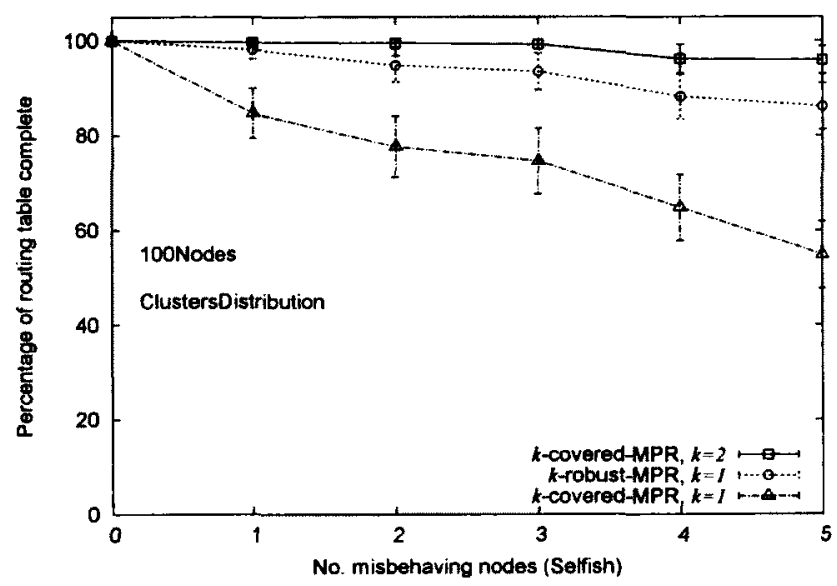

(b) Percentage of nodes with complete routing tables.

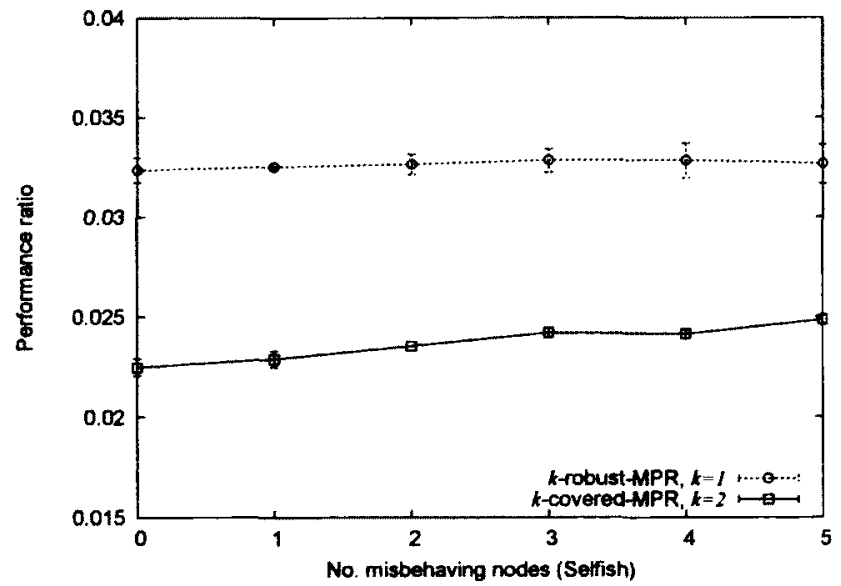

(c) Performance ratio.

Figure 5.4: Comparative of functions $k$-covered-MPR and $k$-robust-MPR under the presence of selfish nodes. 


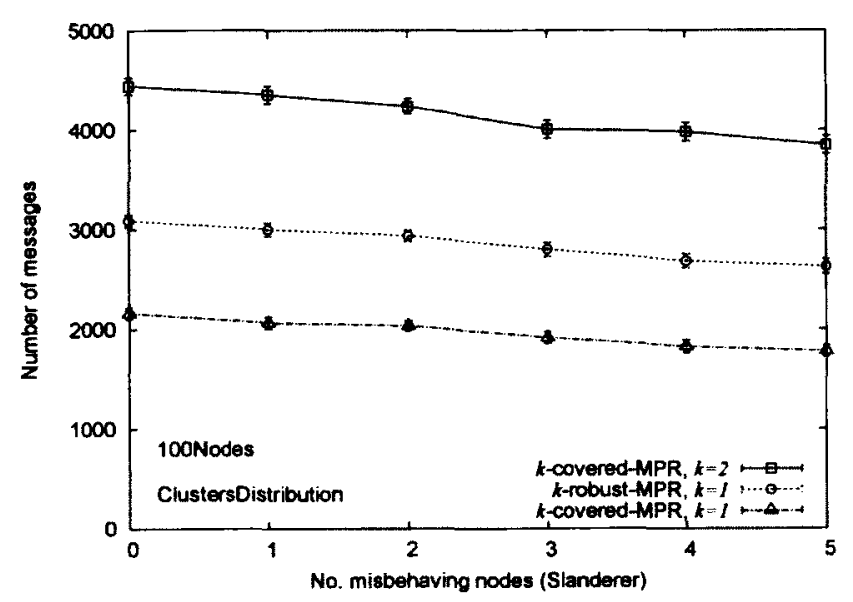

(a) Number of Topology Control messages.

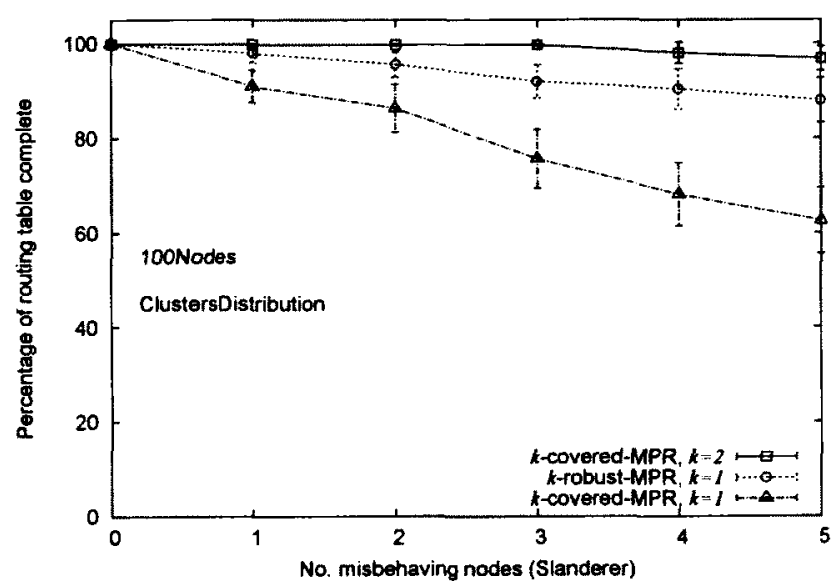

(b) Percentage of nodes with complete routing tables.

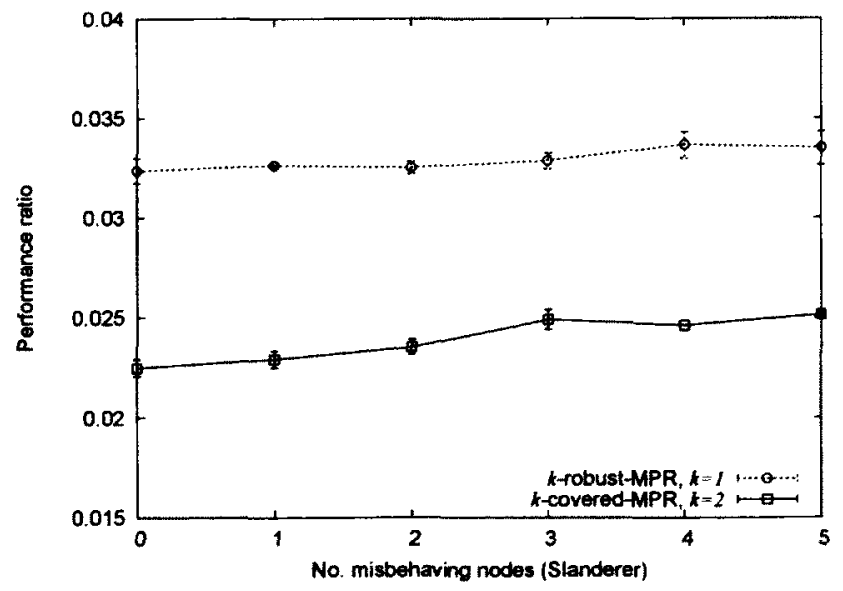

(c) Performance ratio.

Figure 5.5: Comparative of functions $k$-covered-MPR and $k$-robust-MPR under the presence of slanderer nodes. 


\begin{tabular}{|l|l|}
\hline \multicolumn{2}{|c|}{ Simulator Parameters } \\
\hline Propagation model & TwoRayGround \\
\hline Network type & IEEE $802.11(2 \mathrm{Mbps})$ \\
\hline Area & $1000 \mathrm{~m} \times 1000 \mathrm{~m}$ square \\
\hline Transmission Range & $200 \mathrm{mts}$ \\
\hline Number of nodes & 50,100 \\
\hline Nodes' Distribution & Clusters \\
\hline \% Misbehaving nodes & $1-5$ \\
\hline Coverage & $\mathrm{k}=1,2$ \\
\hline Simulations Time & 15 sec \\
\hline Adversaries & Selfish and Slanderer nodes \\
\hline
\end{tabular}

Table 5.2: NS-2 and scenario parameters.

We test functions $k$-robust-MPR and $k$-covered-MPR (with and without additional coverage), against one to five selfish or slanderer nodes. We test one hundred different topologies and one hundred nodes in each case. For each topology, we test all the approaches. In all the topologies, the misbehaving nodes are selected at random among all the MPRs. For our experiments, the nodes are distributed in ten clusters. In [96] authors organize an OLSR network in clusters in a hierarchical architecture. We can consider our topology as a particular set of clusters at the same level. The nodes in each cluster follow a Zipf [39] distribution. Then, following this distribution the nodes are located in the center of each cluster with higher probability. Thus, it is possible to obtain topologies where at least $40 \%$ of the nodes have $k$-robust MPR sets, with $k \geq 1$.

Figure 5.4 depicts the average number of nodes with complete routing tables and $95 \%$ confidence intervals. It shows how our strategy offers additional protection to mitigate the effect of misbehaving nodes in contrast with the selection of MPRs without additional coverage. We point out that is not always possible to find $k$-robust MPR sets for all the nodes in the network. In consequence, if the number of misbehaving nodes increase the level of protection decrease. Notice that our $k$-robust-MPR function mitigates the effect of misbehaving nodes with a better performance than the $k$-covered-MPR (cf. Figure 5.4(c)). Figure 5.5 depicts our results, but with one to five slanderer nodes. Again, we observe that our $k$-robust-MPR function mitigates the effect of misbehaving nodes with a better performance than the $k$-covered-MPR (cf. Figure 5.5(c)). 


\subsection{Conclusion}

In this chapter, we proposed an improved MPR selection with additional coverage (cf., function $k$-robust-MPR) to mitigate control traffic attacks in an OLSR network. Our goal is to provide service availability and security. In our proposal, every node selects, if it is possible, $k+1$ disjoint MPR sets. As a result, we obtain a $k$-robust-MPR set. The RFC3626 defines an MPR selection with additional coverage (cf., function $k$-covered-MPR), however, the number of topology control messages increases considerably reducing the performance of the network. We compared functions $k$-covered-MPR and $k$-robust-MPR in the presence of misbehaving nodes. We measured the number of nodes with complete routing tables after the execution of the OLSR protocol. Our experiments show that our function $k$-robust-MPR reduces the amount of traffic generated by function $k$-coveredMPR, and offers equivalent protection against control traffic attacks. Additionally, our function $k$-robust-MPR increases the performance ratio of the number of nodes with complete routing tables over the number of topology control messages. 


\section{Chapter 6}

\section{Security Issues in Hierarchical OLSR}

\subsection{Introduction}

The Hierarchical Optimized Link State Routing (HOLSR) [96] is a proactive routing protocol designed to improve scalability of heterogeneous MANETs. HOLSR organizes the network in logical levels and distributes the nodes in clusters. In every cluster, it implements the mechanisms and algorithms of the original OLSR [34] to generate and to distribute control traffic information. Nevertheless, HOLSR was designed without security concerns and both inherits and add new security threats. In HOLSR, every node must be able to acquire an accurate topology map to preserve the connectivity in the network. Then, each node has two main tasks to perform: (a) to generate control traffic information or (b) to relay that information on behalf of other nodes. Thus, information contained in Hello and TC messages are used to calculate optimal routes from any given node to any destination within each cluster. Additionally, Hierarchical Topology Control (HTC) messages are implemented to advertise membership information from a cluster to other nodes in higher levels.

As in bare OLSR, the core optimization of the protocol is the selection of MPR as a flooding mechanism for distributing TC and HTC messages to all levels of the hierarchical architecture. In HOLSR, topology map acquisition [31] is the ability of any given node to acquire a complete view of the network connectivity (i.e., routing tables) according to their topological level in the network. A node with an incomplete topological map is unable of calculating routing paths and forwarding data. In this context, a malicious node is defined as a node that interrupts the flooding of control traffic information or does not obey the rules of the protocol to maintain the hierarchical architecture. Topology map acquisition is affected by a malicious node that performs a flooding disruption attack to interrupt the propagation of control information. This attack can be performed by a misbehaving node 
that reports either a false identity (i.e., identity spoofing) or a false link (i.e., link spoofing) to perturb the proper selection of the MPRs. Furthermore, a malicious node might not relay properly control traffic information on behalf other nodes. Thus, the nodes in the network will not be able of constructing a complete map of other nodes attached to its cluster or in lower hierarchical levels. Notice that in some cases flooding disruption attacks can be performed even in a secured HOLSR network(e.g., a node does not forward control traffic information to save energy). Additionally, if an attack is detected, it is necessary to implement an efficient mechanism to advertise other nodes in the network.

Hierarchical routing protocols for MANETs have been analyzed from a performance perspective, but not security. In our work, we analyze flooding disruption attacks that affect the topology map acquisition process in hierarchical networks, i.e., inter-cluster communication. Also, we study the effect of misbehaving node that attempts to unbalance the distribution of nodes during the cluster formation phase. We use HOLSR to present the attacks and our proposed solutions. However, this attacks may affect other OLSR-based hierarchical networks such as: Cluster OLSR (C-OLSR) [88] proposed by Ros et al., the Multi-level OLSR Routing using the Host and Network Association messages Extension (MORHE) [97] presented by Voorhean et al. and the tree-based logical topology $[17,16]$ to provide hierarchical routing presented by Baccelli., are are also affected by flooding disruption and cluster formation attacks presented in this chapter. To mitigate the effect of the attacks, we propos the selection of MPRs with additional coverage, i.e., $k$-Coverd-MPR and $k$-Robust-MPR selections, and a Non-source dependant (NSD) mechanism RFC3626 [34] as strategies to enforce the inter-cluster communication (c.f., Section 6.5). To prevent attacks during the cluster formation phase, we proposed an algorithm based on hash chains to protect mutable fields in topology control messages (c.f., Section 6.6.2). 


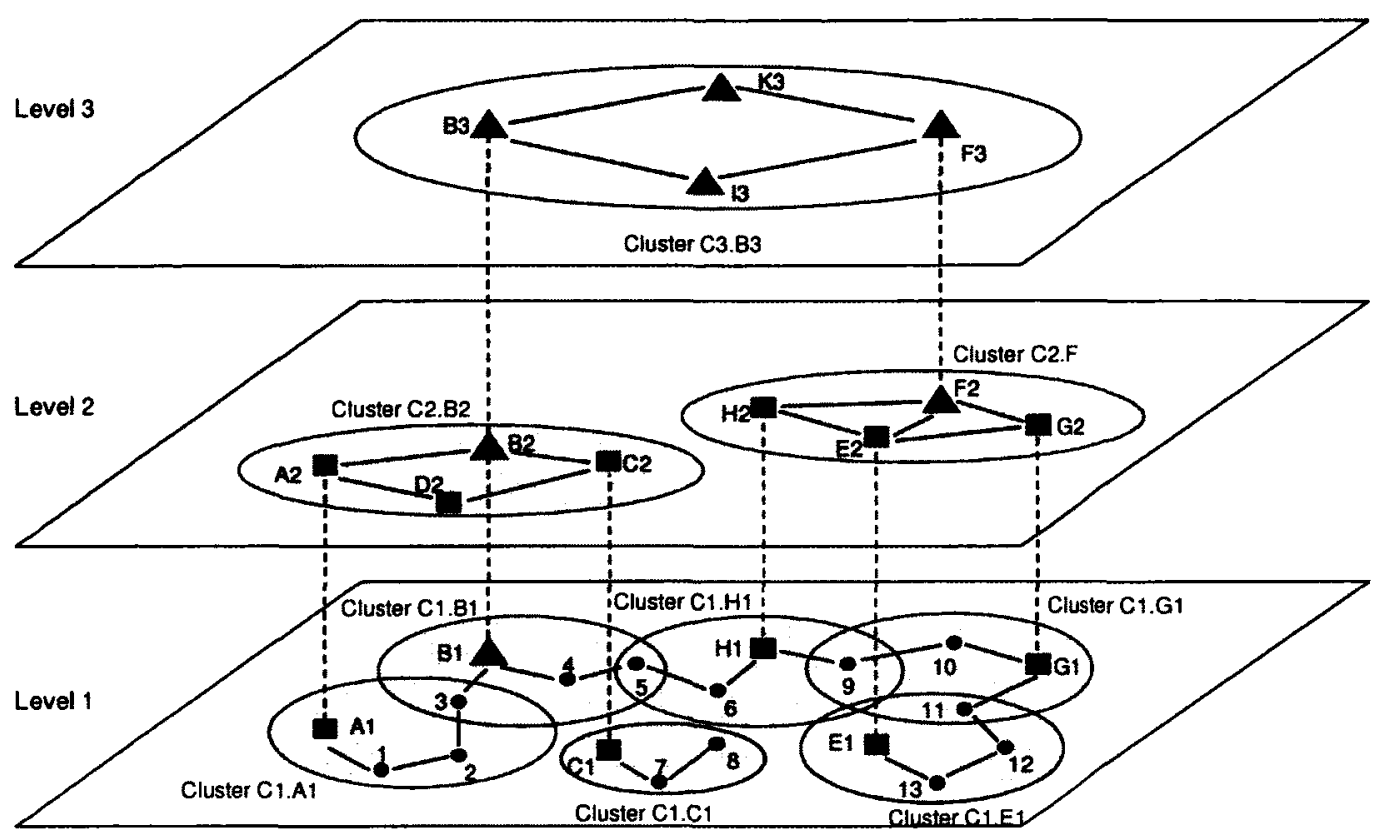

Figure 6.1: Example of a hierarchical architecture with heterogeneous nodes.

\subsection{Hierarchical OLSR (HOLSR)}

MANETs are by nature formed by heterogeneous devices and nodes that can join the network without following a predictable pattern. Furthermore, scalability is a problem in MANETS. Scalability can be defined as the capacity of the network to adjust or to maintain its performance even if the number of nodes in the network increases [96]. OLSR is a flat routing protocol and the performance of the protocol tends to degrade when the number of nodes increases due to a higher number of topology control messages propagated through the network. The MPR mechanism is local and therefore very scalable. However, the diffusion by all the nodes in the network of all the link-state information is less scalable. For instance, in [76] Palma et. al., show that OLSR have good results in terms of scalability in networks with up to 70 nodes, preferably with a moderate node speed and where the number of traffic flows is also moderate. However, OLSR's performance decreases in large heterogeneous ad hoc networks. Additionally, OLSR does not differentiate the capabilities of its member nodes and, in consequence, does not exploit nodes 
with higher capabilities. Thus, HOLSR is an approach designed to improve the scalability of OLSR protocol in large-scale heterogeneous networks. The main improvements are a reduction in the amount of topology control traffic and efficient use of high capacity nodes.

HOLSR organizes the network in hierarchical clusters. This architecture allows to reduce the routing computational cost, i.e., in case a link is broken only nodes inside the same cluster have to recalculate their routing table while nodes in different clusters are not affected. In HOLSR, nodes are organized according to their capacities. A HOLSR architecture is illustrated in Fig. 6.1. At level 1, we have low-capability nodes and one interface represented by circles. Nodes at the topology level 2 are equipped with up to two wireless interfaces, designated by squares. Nodes at level 2 employ one interface to communicate with nodes at level 1 . Nodes at level 3 , designated by triangles, represent high-capacity nodes with up to three wireless interfaces to communicate with nodes at every level. Thus, in Fig. 6.1, node F3 represents node F's interface at level 3. The only restriction for nodes at levels 2 and 3 is that they have at least one interface to communicate with nodes at levels 2 or 3 , respectively. For instance, in Fig. 6.1 node $F$ has two interfaces and can communicate with nodes at levels 2 and 3 . Node $A$ has also two interfaces and establishes communication with nodes at levels 1 and 2 . Node D can just communicate with nodes at level 2 . In the example, the notation used to name the clusters reflects the level of the cluster and the cluster head, e.g., C1.A means that the cluster is at level 1 and the cluster head is node A. HOLSR allows formation of multiple clusters and, unlike OLSR, HOLSR nodes can exchange Hello and TC messages exclusively within each cluster. This constraint reduces the amount of traffic information broadcast to the entire ad hoc network.

\subsubsection{Cluster Formation}

The topology control information between clusters is exchanged via specialized HOLSR nodes designed as cluster heads. The selection of cluster heads and classification of nodes according to their capabilities is defined at the startup of the HOLSR process. A cluster is formed by a group of mobile nodes -at the same hierarchical level- that have selected a common cluster head. Nodes can move from one cluster to another and associate with the nearest cluster head. Any node participating in multiple topology levels automatically 
becomes the cluster head of the lower-level cluster. In HOLSR, a cluster head declares its status and invites other nodes to join it by periodically sending out Cluster ID Announcement (CID) messages. These messages are transmitted in the same packet with Hello messages using a message grouping technique. This technique is implemented to reduce the number of packet transmissions. A CID message contains two fields:

- cluster head: interface address of the originator of the message.

- distance: distance in hops to the cluster head generating the message.

Every time the cluster head generates a CID message, it initializes the field distance to one. The receiver node joins the cluster head and sends a new CID message. The new CID message increases the value of the distance by one unit. This mechanism allows to invite other nodes to join the same cluster. Nodes in an HOLSR network might receive CID messages from different cluster heads indicating that they are located in an overlapping region. In that case, the receiver node will join the closest cluster head (in terms of hops). If a node receives multiple CID messages with the same hop count, then it will attach itself to the cluster head from which it receives the first CID message. The clusters are automatically reconfigured. If the topology changes, nodes will evaluate new CID messages and will attach themselves to a closer cluster head. For instance, in Fig. 6.1 the interface A1 of node A, sends out a CID message with values: cluster head $=\mathrm{A} 1$ and distance $=0$. Then, node 1 receives the message and joins cluster head Al. Next, node 1 generates a new CID message increasing the hop count, i.e. cluster head=A1 and distance $=1$, node 2 receives the messages and also joins cluster head A1. Node 3 , which is in transmission range of both node 2 and cluster head B1, receives two CID messages but decides to join the closest cluster C1.B, managed by $B 1$. If a node receives various CID messages with the same distance value, it will attach itself to the cluster head from which it has received the first message. The nodes will remain attached to the selected cluster head as long as the topology does not change. If it happens, the nodes will process CID messages to find the closest cluster head and attach themselves to a new cluster.

Robustness is ensured thanks to a built-in diagnostic feature. Each node registers a timeout value for the CID messages received. If a cluster head becomes inactive or moves away, 
then the neighbor nodes will stop receiving CID messages. Eventually the CID message timeout will expire and the CID information will become invalid. Thus, nodes will start to process new CID messages from other clusters and will select a new cluster head. For instance, in Fig. 6.1, assuming cluster head B1 went down, then node 3 will join cluster head $\mathrm{Al}$ after receiving a CID message from node 2 , and node 4 will join cluster $\mathrm{C} 1 . \mathrm{H}$ after receiving a message from node 5 . If no CID messages are received, then it means that the network is not partitioned in clusters anymore and behaves following the basic OLSR protocol.

\subsubsection{Cluster Head Message Exchange}

The hierarchical architecture must support the exchange of topology control information between clusters without introducing additional overhead. Thus, HTC messages are generated by the cluster head and used to transmit the membership information of a cluster to higher level nodes. There are three basic types of HTC messages:

- full membership: these messages are periodically transmitted by a cluster head to provide information about its cluster members, including any node in lower levels beneath it.

- update: to provide information about cluster membership changes. The updated HTC are used when a node leaves or joins a cluster.

- request: request HTC messages are used when a packet loss has occurred. HTC messages carry a sequence number field, this field allows a node to request the retransmission of a full membership HTC message.

HTC forwarding is enabled by the MPRs and restricted within a cluster. Nodes at the highest topology level have full knowledge of all nodes in the network and their routing tables are as large as they would be in an OLSR-based network. However, in lower levels, the size of the routing table of every node is restricted to the size of the cluster and it is smaller than in OLSR. For instance, in Fig. 6.1 the cluster head A generates an HTC message for the interface A2 (level 2) announcing that nodes 1,2 and A1 are members of its cluster at level 1 . The message is relayed to all nodes at the same level. Then, node $B$ 


\begin{tabular}{llll}
\hline Messages & Generated by & Retransmitted by & Reported information \\
\hline Hello & Every node & N/A & One-hop neighbors \\
TC & MPRs & MPRs & MPR Selector Set \\
CID & Cluster heads & N/A & Cluster head Identification \\
HTC & Cluster heads & MPRs & Nodes within a cluster \\
\hline
\end{tabular}

Table 6.1: Summary of control traffic messages in HOLSR.

generates an HTC message for the interface B3 (level 3) advertising that nodes 1, 2, 3, 4, $5,7,8, \mathrm{~A} 1, \mathrm{~B} 1, \mathrm{C} 1$ (at level 1) and A2, B2, C2, D2 (at level 2) are members of its cluster. Table 6.1 presents a summary of the messages implemented in HOLSR.

\subsubsection{Topology Control Propagation}

Nodes in each cluster at different levels select their MPRs to flood control traffic information. Control messages are generated and propagated exclusively within each cluster, unless a node is located in the overlapping zone of several clusters. For example, in Fig. 6.1 node 2 is within the border of cluster C1.A and may accept a TC or HTC message from node 3 located in cluster C1.B. However, node 2 retains the information without retransmitting it to its cluster. Thus, except for the border nodes, knowledge of nodes about the cluster is restricted to the cluster itself. Data transfer between nodes in the same cluster is achieved directly via the information in the routing tables. However, when transmitting data to destinations outside the local scope of a cluster, the cluster heads are always used act a gateway mechanism by member nodes at lower hierarchal levels. When transmitting data between border nodes in different clusters at the same level, a different strategy might be used. In this situation, the cluster head is not used as a gateway to relay the information, and nearby nodes in different clusters at the same topology level can communicate directly without having to follow the strict clustering hierarchy. This means that, data transfer between nodes is achieved following three different strategies:

- communication between nodes in the same cluster is achieved via the routing information in their routing tables,

- data transfer between nodes in different clusters is achieved through the cluster heads as a gateway mechanism, but 
- if the nodes are neighboring nodes in different clusters at the same topology level, the cluster heads are not used and data packets are directly relayed.

Therefore, HOLSR offers two main advantages (a) the traffic control messages reflecting local movement are restricted to each cluster (thus, reducing the routing table computation overhead), and (b) an efficient use of high-capacity nodes without overloading them.

\subsection{Other Hierarchical Approaches}

In this section, we review other hierarchical models based on OLSR to improve scalability in MANETs.

\subsubsection{Cluster OLSR}

In [88], Ros et al. present the Cluster OLSR (C-OLSR) protocol. Unlike HOLSR, COLSR does not assume any particular cluster formation algorithm nor existence of higher capacity nodes. C-OLSR implements OLSR inside every cluster and uses the MPR mechanism for distributing control traffic at both inter-cluster and intra-cluster levels. C-OLSR limits the forwarding of TC messages inside every cluster to minimize the number control traffic messages. Every node can compute routes to any other node inside its cluster. To reach nodes in other clusters, nodes create routes to every cluster and not to every node. When a data packet arrives to a destination cluster, every node has enough information to deliver the packet to its final destination. This mechanism reduces the size of the routing tables.

For inter-cluster communications, Cluster Hello (C-Hello) and Cluster Topology Control (C-TC) messages are defined. C-Hello messages are used to sense neighboring clusters and to compute the Cluster MPR (C-MPR) set. C-Hello messages are flooded within the receiver cluster but not retransmitted to neighbor clusters. A C-MPR is a cluster selected to reach other clusters and mitigate the overhead of distributing C-TC messages for intercluster communications. C-TC messages advertise the nodes within a cluster to all the network. Fig. 6.2, shows an example of a C-OLSR network. At the first level, nodes are 


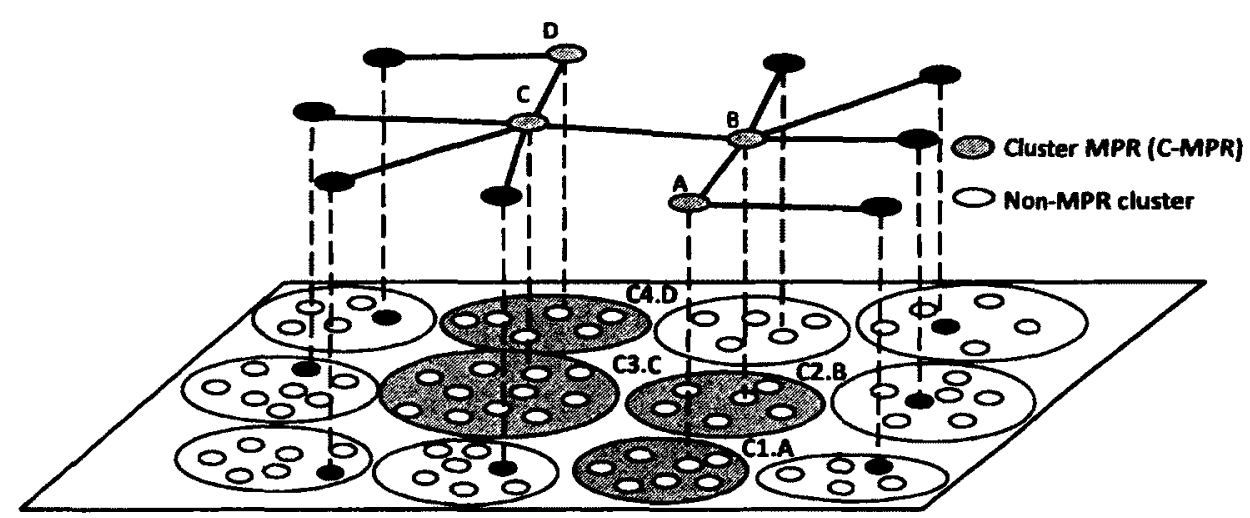

Figure 6.2: Example of a Cluster OLSR network. Consider gray clusters as C-MPRs.

organized in clusters. The second level, shows how clusters are linked. Gray clusters are C-MPRs, e.g., C1.A is a C-MPR and node $A$ is the cluster head. When a node in a cluster needs to send a data packet to a node inside another cluster, it computes a path through the clusters selected as C-MPRs, i.e., $C 1 . A, C 2 . B, C 3 . C$ and $C 4 . D$.

When a C-Hello or C-TC messages arrive to a cluster, they are relayed to every node in the cluster. This allows nodes to learn about clusters topological information. C-TC messages must be relayed to adjacent clusters, only if the sender of the message has selected the receiver node as an C-MPR. To support this hierarchical architecture, every C-OLSR node has additional information repositories: one-hop neighbor cluster set, two-hop neighbor cluster set, cluster topology set, cluster MPR set and cluster MPR selector set. The information in these repositories supports inter-cluster communications. In C-OLSR, not every node has to generate inter-cluster information. The generation of C-Hello and C-TC messages can be done according to three different algorithms: a cluster head-based algorithm, a distributed algorithm or a hybrid approach. In the former case, only cluster heads generate control information. In the second algorithm, topology information is generated exclusively by border nodes. Finally, in the hybrid approach, C-Hello messages are generated by border nodes and C-TC messages are generated by the cluster heads. In all cases, the selected C-MPRs are responsible for forwarding C-TC messages. 


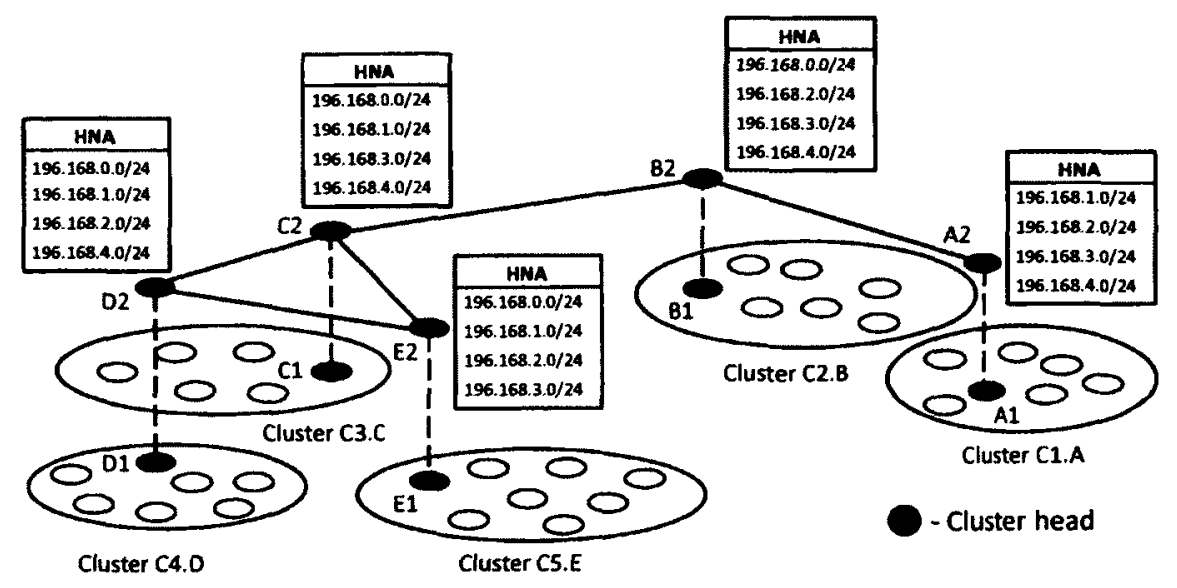

Figure 6.3: Example of a MORHE network. Consider black nodes as backbone nodes.

\subsubsection{The Multi-level OLSR Routing Using the HNA Extension}

In [97], Voorhaen et al. present a multi-level routing scheme for ad hoc networks based on OLSR. Multi-level OLSR Routing using the HNA Extension (MORHE) protocol improves scalability by exploiting high capability nodes. Using HNA messages and hierarchical addressing, MORHE constructs an overlay network formed by nodes with higher capabilities. Nodes with higher capabilities are selected as cluster heads. A cluster head is called a backbone node. Backbone nodes are chosen before network deployment and have more than one interface. Nodes are organized into clusters around every backbone node. Fig. 6.3, shows an example of a two-levels MORHE network. Nodes $A, B, C, D$ and $E$ are backbone nodes. Backbone nodes use one interface to communicate with the nodes inside their cluster and the second interface for inter-cluster communications. For instance, backbone node $A$, communicates with the nodes at the first level through the interface $A 1$ and uses interface $A 2$ to communicate with other backbone nodes. OLSR is implemented at each level.

MORHE is similar to HOLSR, nonetheless it only uses HNA messages already defined in the RFC 3626 [34]. Each backbone node periodically sends HNA messages informing other backbone nodes that it can reach all the nodes in the subnet that it is connected to. When a backbone node receives a HNA message, it updates its association database. Every backbone node uses HNA messages to inform all the nodes in its cluster about other 
clusters that can be reached. HNA messages are distributed using the MPR mechanism as defined in OLSR. Nodes can communicate directly with every node inside its cluster. Backbone nodes enable communication between nodes in different clusters. When a packet arrives at a backbone node, it attempts to find a route to the destination in its cluster. If this fails, then the backbone node retransmits the message to another backbone node. If the receiver finds a route, then it forwards the packet inside its cluster. In a MORHE network, every cluster is identified as a subnetwork. For instance, in Fig. 6.3, the network is divided in five subnetworks. Every backbone node has the IP addresses of every subnetwork in its association table. For example, 192.168.1.0/24 is the prefix of an IPv4 subnetwork, having 24 bits allocated for the network prefix, the remaining 8 bits are reserved for host addressing. If a node inside the subnetwork 192.168.0.0/24 needs to communicate with a node in the subnetwork 192.168.2.0/24, then it sends the packet to its backbone node which retransmits the packet to its final destination.

\subsubsection{Tree Clustering}

In [17, 16], Baccelli proposed a Tree Clustering mechanism to enable hierarchical routing within an OLSR-based network. Each cluster is a tree. Their head is the root. To organize the network in trees, every node selects as its parent the adjacent node with the maximum number of one-hop neighbors. The parent of a node is called a node's preferred neighbor. A node with maximum degree, i.e., maximum number of neighbors, is selected as the root of the three. The network is then viewed as a forest, i.e., a collection of logical trees. To form and maintain trees, OLSR nodes periodically exchange Branch messages. These messages are piggy-backed with Hello messages. Branch messages are not retransmitted. Within a Branch message, a node specifies its identity, the tree it belongs to, its parent in the tree and its distance in hops to the root. Roots can choose to limit the size of their three by imposing a maximum depth value. The organization in trees is dynamic. A mechanism allows to switch between a traditional flat networking, i.e., flat mode or a hierarchical networking, i.e., tree mode. The mechanism to transit between the flat mode and the tree mode is explained in detail in [17]. 


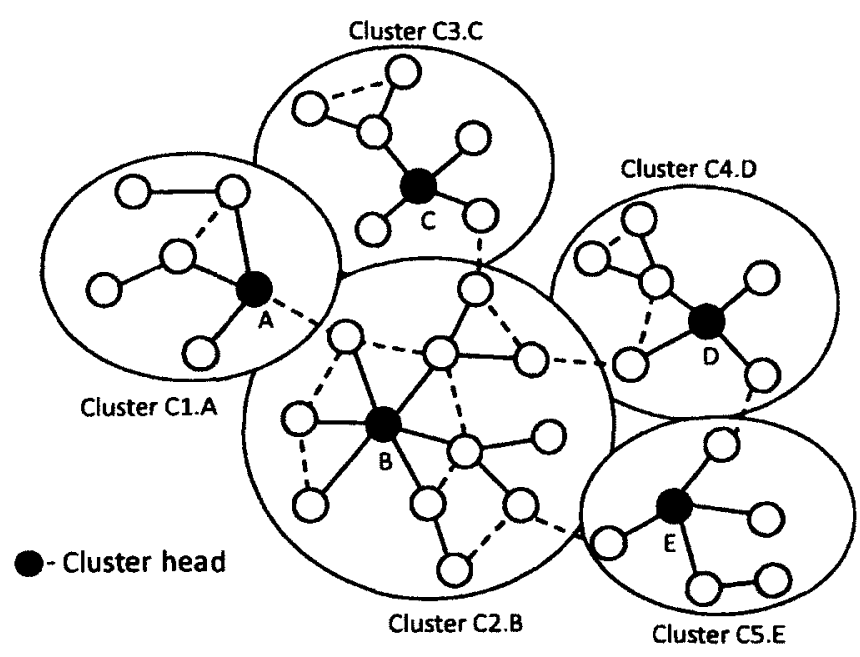

Figure 6.4: Tree clustering. Black nodes represents the roots of the tree. Branches of the trees are shown with solid lines between nodes. Links that are not branches are dashed.

Within a tree, OLSR nodes operate as if there was no tree, except that messages originated by a node in a different tree are not considered and not forwarded, the root is responsible for the communication between the tree and the rest of the network, and a node in contact with another tree i.e., a leaf node, must inform its entire tree (specially its root), of the distance to reach other roots. A leaf node must generate a Leaf message for each other tree it reaches. In a Leaf message, the node specifies its ID, the root of the neighbor tree and the estimated distance between the roots, i.e., the sum between its depth in its tree, and the distance to the root of the neighbor tree. With this information, every root is able to compute the shortest path to reach its neighbor roots.

This protocol employs Hello and TC messages within every tree, but implements Super-Hello (S-Hello), Super-TC (S-TC) and Super-HNA (S-HNA) messages for intercluster communications. Super messages are generated exclusively by the roots. These messages are identical to regular messages except for an additional field that includes the IP address of the next root to reach. Unlike regular messages, Super-messages are routed using the constructed paths instead of being flooded. Super-messages are unicasted using the shortest root-to-root path advertised by Leaf messages. Super-messages are the only messages to be forwarded outside a tree. MPR selection is performed as if there were no trees. When a tree mode is activated, the scope of TC messages is limited to the tree they 
were generated. However, Super-messages are forwarded between clusters following the MPR flooding mechanism.

To allow hierarchical routing, routes exchange Super-messages in order to identify other roots and construct a Super-topology. S-Hello and S-TC messages allow the roots to construct a super-topology formed by roots. The roots periodically exchange S-Hello messages to learn about other roots in neighbor trees (i.e., one-super-hop neighbors). As in OLSR, every root computes its super-MPR set formed by other roots. A super set of MPRs is used for distributing S-TC messages among clusters. S-Hello messages are not forwarded. S-TC messages are forwarded by the S-MPRs. S-TC messages include the super-selector set, i.e., the roots that have selected the sender as a S-MPR. Finally, every root generates S-HNA messages to inform other roots about the link state information within its cluster. Therefore, every root is aware of the link state information of other threes. Routing among clusters is achieved using the information between S-TC and SHNA messages. Traffic outside the tree scope is achieved via the root nodes. Fig. 6.4 shows an example of a tree clustering hierarchical architecture. Nodes $A, B, C, D$ and $E$ are selected as roots. These nodes have the maximum degree. Root node $A$ selects $B$ as its MPR to reach root trees $C, D$ and $E$. When a node inside cluster C1.A needs to communicate with a node inside cluster C5.E, it sends the data traffic to its root node $A$ which retransmits the traffic to its final destination trough $B$ and $E$.

Table 6.2 presents a summary of the features of each hierarchical approach that we

\begin{tabular}{|c|c|c|c|c|}
\hline $\begin{array}{l}\text { Routing } \\
\text { Protocol }\end{array}$ & Network & $\begin{array}{l}\text { Logical } \\
\text { Levels }\end{array}$ & Messages & $\begin{array}{l}\text { Cluster Formation Algo- } \\
\text { rithm }\end{array}$ \\
\hline HOLSR & Heterogeneous & $\mathbf{n}$ & CID and HTC & Yes \\
\hline MORHE & Heterogeneous & $\mathrm{n}$ & HNA & No \\
\hline C-OLSR & Homogeneous & 2 & C-Hello and C-TC & No \\
\hline Tree & Homogeneous & 2 & $\begin{array}{l}\text { Leaf, Branch, S-Hello, S-TC } \\
\text { and S-HNA }\end{array}$ & Yes \\
\hline
\end{tabular}

Table 6.2: Comparison of OLSR-based hierarchical approaches. All approaches implement Hello and TC message for intra-cluster communications. 
reviewed. Unlike MORHE and C-OLSR, HOLSR and the Tree clustering approaches include a cluster formation mechanism. MORHE and HOLSR were designed for heterogeneous networks and multiple hierarchical levels. C-OLSR and Tree clustering were designed for homogeneous networks and two hierarchical levels. Nevertheless, these approaches might be implemented in networks with heterogeneous capabilities. All approaches implement the MPR mechanism for distributing control traffic messages.

\subsection{Flooding Disruption Attacks in HOLSR}

The flooding mechanism for control traffic information in HOLSR is based on the correct selection of the MPRs. Control traffic messages (i.e., TC and HTC messages) are forwarded exclusively by the MPRs. An attacker seeking to interrupt the control traffic flooding can either (a) manipulate the information about the one and two-hop neighbors of a given node to cause the MPR selection to fail, or (b) misbehave during the generation and forwarding processes. Thus, a node will receive incomplete information about other nodes in its cluster or in lower level clusters. The attack has a cross layer impact if the affected node is a cluster head with an interface to an upper level. In this case, nodes in the upper level will fail to compute a route to nodes in lower levels of the network. For instance, consider in Fig. 6.1 that node E2 selects node H2 as its MPR, nonetheless $\mathrm{H} 2$ misbehaves and does not retransmit any control traffic message. In consequence, node F2 and nodes in cluster C3.B will not be aware of nodes within cluster C1.E.

\subsection{Countermeasures}

In HOLSR, the MPR selection reduces at minimum the overhead generated by control traffic messages, if every node selects its MPR set with the following conditions: (i) the MPR set is kept at minimum, (ii) an MPR retransmits control traffic messages if and only if the sender node is included in its selector table, and (iii) only partial link state information is transmitted, i.e., an MPR reports only links with its selector nodes. Nevertheless, we can loosen up the previous restriction in order to offer a higher level of security while maintaining a trade-off between security and performance. In the following subsections, we 
describe a set of strategies to reduce the effect of flooding disruption attacks. The strategies that we describe are based on the selection of MPRs with additional coverage, generation of TC messages with redundant link state information and a non source-dependent forwarding mechanism.

\subsubsection{MPRs with Additional Coverage}

Additional coverage in the selection of the MPRs is defined in [34], as the ability of a node to select redundant MPRs. In Chapter 5, we reviewed the selection of the MPRs with additional coverage for flat OLSR. The selection of MPRs with extra coverage is defined in the RFC3626 [34], we named this approach a $k$-Covered-MPR set. However, the overhead generated by the excessive number of TC and HTC messages reduces the performance of the network. This problem is addressed with an improved $k$-Robust-MPR selection presented in [23] and detailed in Chapter 5. The $k$-Robust-MPR set has two main properties: (a) in a $k$-Robust-MPR set it is possible to discard a maximum of $k$ MPR sets, and the remaining set it is still a valid MPR set, and (b) if we can only find $k^{\prime}+1$ disjoint MPR sets, such that $k^{\prime}+1$ is less or equal than a value of $k$, we obtain a valid $k^{\prime}$-robust-MPR set. This improved MPR selection balances security and traffic overhead.

In HOLSR the MPR selection algorithms with or without additional coverage remain the same. Then, the approaches presented in Chapter 5 can be applied in HOLSR. The selection of MPRs for intra-cluster or inter-cluster communication, must be as small as possible to reduce the overhead generated by flooding the network with topology control messages. Nevertheless, additional coverage allows a node to advertise its presence to more nodes within a cluster and helps a cluster head to advertise the nodes in its cluster to other nodes at the same or upper levels. In this manner, extra coverage helps to maintain the integrity of the hierarchical architecture in spite of the presence of malicious nodes during the execution of the protocol.

Figure 6.5 shows an example of $k$-Robust-MPR selection with a value of $k$ equal to one. Node $i$ can select either $\{g\}$ or $\{f, j\}$ as valid disjoint MPR sets, then node $i$ can compute a 1-Robust-MPR set formed by $\{g, f, j\}$. Then, if node $g$ misbehaves, node $i$ can 
discard it and the subset $\{f, j\}$ remains as a valid MPR set.

\subsubsection{Redundant Information}

In contrast to other classic link state protocols, such as the OSPF [69], in HOLSR only partial link state information is diffused. Periodically, an MPR generates TC messages reporting only nodes in its selector table to calculate optimal routes to every destination. However, the advertised link set of a node may include links to neighbor nodes which are not in the MPR selector set of the node. The minimal set of links that any MPR must advertise in its TC messages are the links to its MPR selectors. Nevertheless, the advertised link set may include links to the whole neighbor set of the node. The diffused link-state information can be tunned through the TC_Redundancy parameter defined in the RFC3626 [34], Section 15.

The parameter TC_Redundancy is local to every node and determines the amount of information that should be included in the TC messages. If the TC_Redundancy parameter is equal to zero, then the advertised link set of the MPR is limited to its MPR selector set. If the TC_Redundancy parameter is equal to one, then the MPR will advertise its MPRs and its MPR selector set. Finally, if the parameter is equal to two, then the MPR will report all its one-hop neighbors. For instance, in Fig. 6.5 node $a$ selects node $\{b\}$ as its only MPR. However, suppose node $c$ misbehaves and reports a false link to node $d$ and a phantom node $x$, node $a$ can not select disjoint MPR sets and will select node $c$ as its only MPR set. If node $c$ does not generate or forward control traffic, then node $a$ will remain isolated. Notice that node $b$ is selected by node $d$ as its MPR, then it reports in its TC messages node $d$ as its only selector node. If node $b$ sets its TC_Redundancy parameter equal to three, then it will report all its one-hope neighbors, including node $a$. As a result, the size of the TC message will increase but this strategy might be used to prevent flooding disruption attacks.

\subsubsection{Non-Source Dependent Mechanism}

In HOLSR, an MPR retransmits a control traffic message (TC or HTC message) following a Source Dependent (SD) strategy, i.e., an MPR forwards a control traffic message if and 
only if the sender of the message is included in its selector table. This mechanism allows to minimize the number of retransmissions and overhead generated by excessive TC messages in the network. In [62], Macker et al. analyze the overhead generated by a nonsource dependent MPR (NSD-MPR) mechanism to support simplified multicast IP routing in MANETs. Nonetheless, this approach can be used to enforce security in HOLSR. In order to avoid an excessive overhead, the mechanism can be useful to retransmit exclusively HTC messages according the following algorithm for a given node $n$ :

- If node $n$ receives an HTC message and node $n$ 's selector table is not empty then process and forward the message. Otherwise, just process the message.

- If node $n$ receives a TC message and node $n$ 's selector table is not empty and the sender of the message is included in node $n$ 's selector table then process and forward the message. Otherwise, just process the message.

For instance, in Fig. 6.5 consider node $a$ as a cluster head and can not select disjoint MPR sets. Suppose, node $c$ misbehaves and reports a false link to node $d$ and a false link to a phantom node $x$. Then, node $a$ is forced to select node $c$ as its only MPR. Node $c$ generates TC messages and announces node $a$ as it selector node but it does not retransmit HTC messages generated by node $a$. In consequence, all nodes reported by node $a$ in its HTC messages will not be advertised by other nodes in its cluster and in upper levels. However, if node $b$ is selected by node $d$ as its MPR and it follows a non-source dependent strategy to retransmit HTC messages, node $a$ 's messages will be retransmitted by node $b$ even if node $a$ is not included in its selector node.

\subsubsection{Future Work: Locally Proactive HOLSR}

In HOLSR, every node must follow the same rules of the protocol even if they have different capabilities, for instance, every node selects its MPR set following the same algorithm without considering its hierarchical level. Nevertheless, in some situations some nodes might follow a different behavior in order to avoid malicious attacks. Therefore, in a Locally Proactive HOLSR (LP-HOLSR) network, a node uses local information to determine the best mechanism to enforce the neighbor discovery process, MPR selection and 


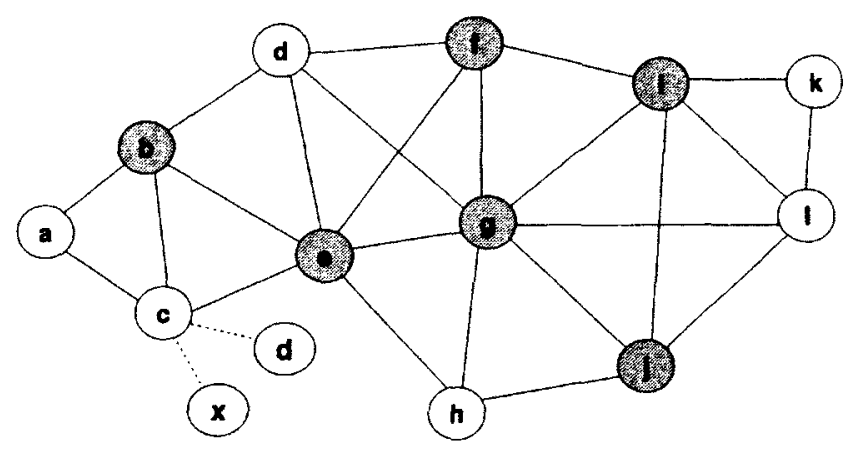

Figure 6.5: Flooding disruption due to link spoofing attack (phantom node).

propagation of link-state information. In [89], Shaukat et al. propose a locally proactive protocol for OLSR, to generate control information using a time interval based on its local network conditions. Thus, according to local parameters the nodes generate control traffic information with more or less frequency. Their goal is to maximize the packet delivery ratio and minimize the control overhead. The authors, however, do not use this approach to enforce security measures.

An LP-HOLSR network can be implemented to prevent an incorrect map acquisition due to flooding disruption attacks. Thus, a node can use local information (i.e., topological level, network density, selector table size, number of interfaces, etc.) to increase the frequency of its topology control messages, to select its MPR set with additional coverage or to modify its forwarding policy in order to enforce the proper flooding of control traffic messages. Notice that nodes with different MPR selection mechanism or forwarding policies can coexist in an LP-HOLSR network. For instance, in Fig. 6.5, node $i$ can select a 1-Robust-MPR set formed by nodes $\{\mathrm{f}, \mathrm{g}, \mathrm{j}\}$ and node $b$ can forward control messages (HTC) following a non-source dependent mechanism.

\subsubsection{Experiments}

We conducted simulations to assess the effectiveness of our proposed countermeasures against flooding disruption attacks in HOLSR networks. We count the number of nodes in a HOLSR network that are able to build complete routing tables under the presence of one to four malicious nodes. We obtain as performance ratio, the percentage of nodes with 


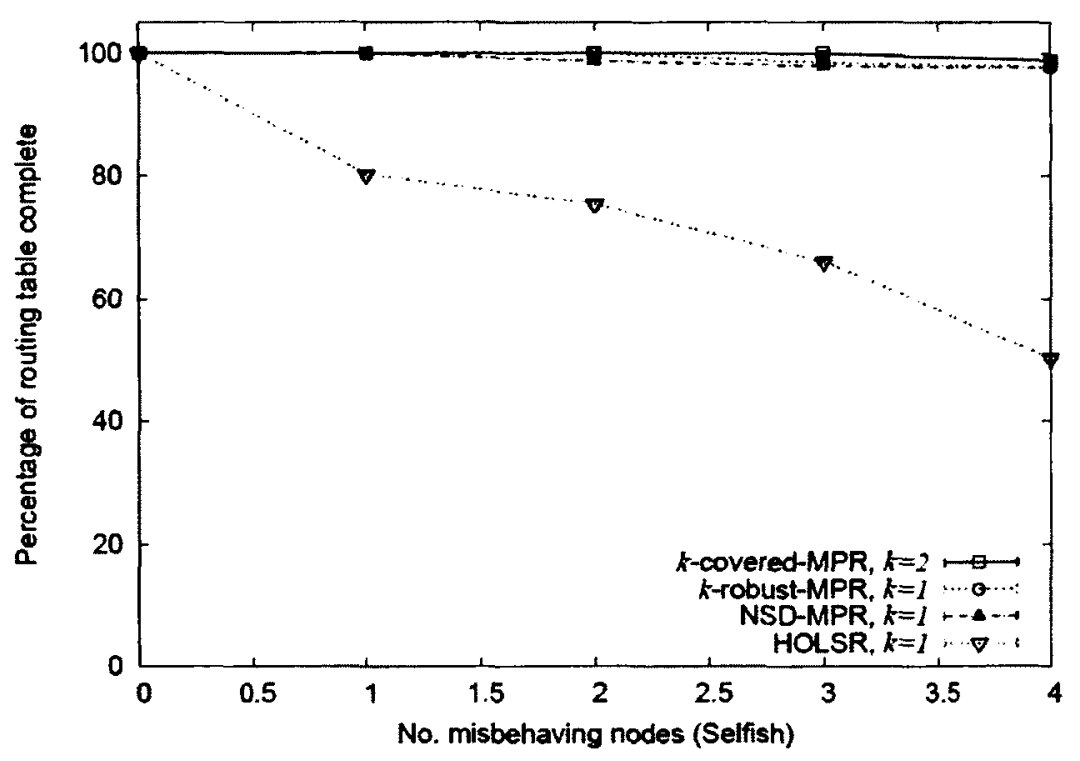

(a) Percentage of nodes with complete routing tables.

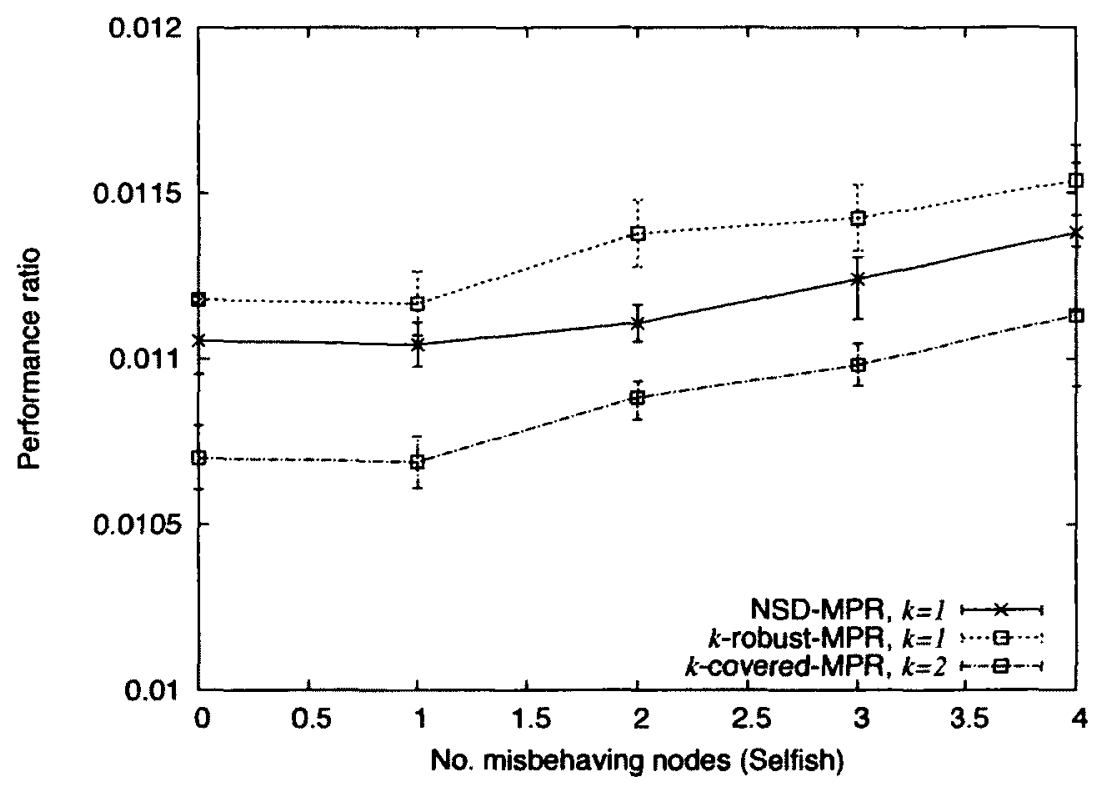

(b) Performance ratio.

Figure 6.6: Comparison of functions NSD-MPR, $k$-covered-MPR, and $k$-robust-MPR under the presence of selfish nodes.

complete routing tables over the number of messages generated during the simulation. We conducted our experiments using the NS-3 simulator [40], version 3.9. We modified the original OLSR code developed by Ros and Carneiro to implement the hierarchical 
approach (i.e., HOLSR) and the countermeasures described in Section 6.5.The malicious nodes are selected among the MPRs, they do not collude to perform an attack, no data traffic is generated and all the scenarios are static.

We test our proposed countermeasures in HOLSR networks with three levels and two hundred nodes in each case: 175 nodes with one interface and a transmission range of 100 $\mathrm{m}, 20$ nodes with up to two interfaces and a transmission range of $200 \mathrm{~m}$, and five nodes with up to three interfaces and a transmission range of $500 \mathrm{~m}$. The nodes with just one interface at the first level, are placed following an uniform distribution. We assume that the administrator of the network can decide the best criteria to distribute the cluster heads. Figure 6.6(a) depicts the average number of nodes with complete routing tables and $95 \%$ confidence intervals. It shows how our strategies offer additional protection to mitigate the effect of selfish nodes in contrast with the selection of MPRs without additional coverage. Notice that the $k$-robust-MPR function mitigates the effect of misbehaving nodes with a better performance than the $k$-covered-MPR and NSD-MPR approach (cf. Figure 6.6(b)). Similar results are expected for the other two cases described in Section

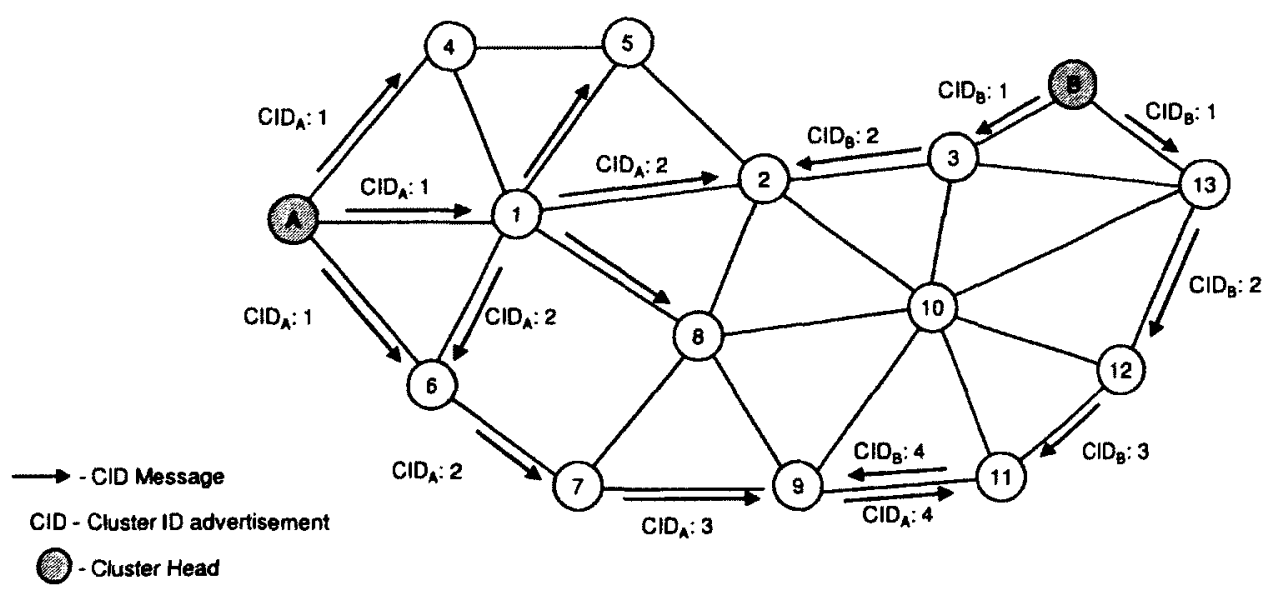

Figure 6.7: CID messages propagation. 


\subsection{Cluster Formation Attack against the HOLSR protocol}

HOLSR has two phases: i) cluster formation and ii) topology map acquisition. In the first phase, HOLSR organizes the network in logical levels and nodes in clusters. Nevertheless, HOLSR is not secure by design. Therefore, the cluster formation phase is also vulnerable to malicious attacks. During the first stage, every cluster head advertises itself through the periodical generation of CID messages that invites other nodes to join. Every CID message has a hop count field that indicates the distance to the originator. The cluster head field of a CID messages points to the originator. When the receiver node joins a cluster head, it generates a new message increasing by one the hop count field. When a node receives messages from different cluster heads, it joins the closest cluster head, in terms of hops. When a node receives CID messages from multiple cluster heads, but with the same hop count, it attaches itself to the cluster head from which it received the first message and remains with that cluster head until the topology changes. As a consequence, a node at the border of different clusters only accepts control traffic information from its cluster.

Fig. 6.7 shows an example of the cluster formation process. Nodes A and B are cluster heads and generate $\mathrm{CID}$ messages $\left(C I D_{A}\right.$ and $C I D_{B}$ respectively). The one-hop neighbor nodes join the originator of the message and generate a new message increasing by one the hop count field. Notice that node 2 receives CID messages from $C H_{A}$ and $C H_{B}$ with the same hop count value. In this case, node 2 chooses the cluster from which it has received the first message. In the same figure, node 9 joins cluster head $A$ and generates a new CID message with hop count equal to four. Node 11 rejects that message because cluster head $B$ is only three hops away. We refer to neighbor nodes in different clusters, such as nodes 9 and 11, as border nodes. Every node registers a timeout value for each CID message received. When a cluster head becomes inactive or moves away, then each neighbor node stops receiving CID messages. Eventually the CID message timeout expires and the CID information becomes invalid. Thus, each node starts to process new CID messages from other clusters and selects a new cluster head. For instance, in Fig. 6.7, assuming cluster head $C H_{A}$ went down, then all nodes attached to it will join cluster head $C H_{B}$ after receiving new CID messages from that cluster head. If no CID messages are received, then it means that the network is not partitioned in clusters anymore and behaves as the original 
OLSR protocol.

An attacker might unsettle this process by generating CID messages with an invalid hop count field. This attack, has a higher impact when the hop count field value is drastically reduced. The receiver nodes may join a cluster head which is farther away than it appears. As a result, the affected cluster head may be overloaded due to an unbalanced node distribution. Additionally, the nodes in some clusters have to include more elements in their routing tables adding unnecessary overhead to the cluster. We handle this risk by implementing a mechanism that implements hash chains to protect the hop count field in every CID message. Our solution is based on the work of Hong et al. in [44]. They present a wormhole detective mechanism and an authentication protocol to strengthen the neighbor relationship establishment in standard OLSR. We address a different kind of attack in HOLSR. Our mechanism protects the integrity of CID messages and enforces the uniform distribution of nodes in every cluster.

\subsubsection{Adversary Model}

The flow of CID messages is an important vulnerability target. The hop count has to be updated every time a new message is retransmitted. Thus, a malicious node might alter this field to unsettle the cluster formation process. The attack, has a bigger impact when the malicious node drastically reduces the hop count field. This is because the receiver nodes accept the CID message with the lowest hop count value. Thus, when an attacker increases drastically the value, the receiver nodes automatically discard the altered message and accept the valid message from other nodes, as this is described in Section 6.2.1. If a node that generates a CID message reinitializes the value of the field hop count, the receiver nodes may join a farther cluster head and discard valid CID messages from closer cluster heads. Then, we only need to address the case when the hop count field is maliciously reduced.

In general, if an attacker is at distance $d$ (in hops) from a cluster head $C H_{i}$, and generates a new CID message with hop count value $j$, the nodes with hop count greater or 


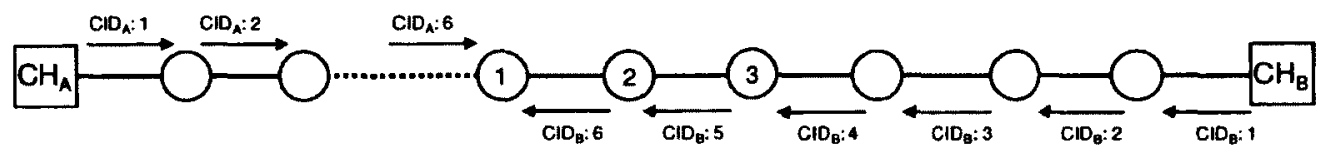

a) Correct CID message propagation.

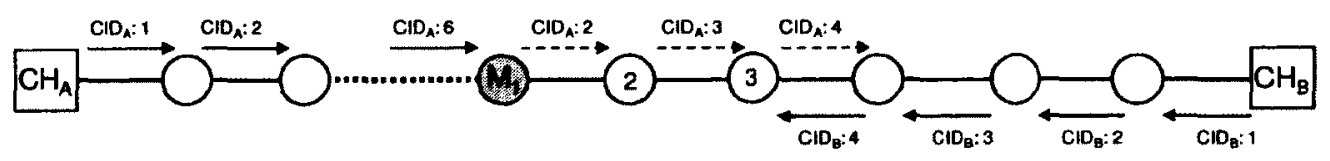

b) Incorrect CID message propagation, decreasing the hop count value.

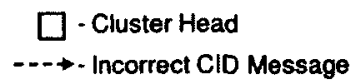

Figure 6.8: CID message attack.

equal to $j+\frac{d}{2}$ from the $C H_{i}$ are potentially affected. For instance, Fig. 6.8 (a) shows the correct propagation of CID messages. Fig. 6.8 (b) shows an example of the attack. In Fig. 6.8 (b), $M_{1}$ is a malicious node at distance six hops from cluster head $C H_{B} . M_{1}$ receives CID messages from $C H_{B}$ and $C H_{A}$, and generates a new CID message assigning an incorrect value to the field hop count, i.e., hop count is set to two. Thus, all nodes at distance greater or equal to four hops (nodes 2 and 3 ) process the message and incorrectly join $\mathrm{CH}_{A}$. Notice that the lowest value that can be used to reinitialize the field hop count is two because CID messages with field hop count equal to one are generated exclusively by the cluster heads. Additionally, we consider that the attacker only has one interface, it can not impersonate a cluster head and it only modifies the hop count value. In the following section, we present our proposed solution to handle this problem.

\subsubsection{Handling the Attack with the use of Hash Chains}

We describe in this section a security improvement over HOLSR based on the use of hash chains [91]. Authentication and integrity is achieved by using hash key chains. For instance, in [57], Lamport proposes a method of user password authentication based on a secure one-way hash function. We do not attempt to address authentication, but the integrity of the messages. A one-way hash chain is based on a one-way hash function $h$ that 
is applied $n$ times to a unique value $x$. Hash functions are relatively easy to compute and can be applied to a block of data of any size.

A hash function can be applied to a block of data of any size and produce a fixed-length output. According to [91], a strong one-way hash function $h$ must have the following properties:

1. The one-way property implies that for any given value $h(x)$, it is infeasible to find the value of $x$.

2. The weak collision resistance property implies that for any given block $x$, it is computationally infeasible to find $y \neq x$ such that $h(x)=h(y)$.

3. The strong collision resistance property implies that it is computationally infeasible to find a pair $(x, y)$ such that $h(x)=h(y)$.

These properties are explained in detail in [91]. Our scheme prevents the attack presented in Section 6.6 while avoiding the use of computationally expensive cryptographic operations. We use the following notation:

- $s_{j}$ : is a random value (i.e., a nonce) generated and known exclusively by the cluster head $\mathrm{CH}_{j}$.

- $h(x)$ : is a strong one-way hash function applied to $x$.

- $h^{n}(x)$ : is a hash chain constructed by applying $n$ times the hash function $h$ to $x$, $h^{n}(x)=h(\ldots h(h(x)))$.

- $t$ : is the maximum number of times that a hash function is applied to $x$.

- $\operatorname{Max}_{j}$ : is the value obtained by applying $t$ times the hash function to a nonce $s_{j}$, $\operatorname{Max}_{j}=h^{t}\left(s_{j}\right)$.

- $i$ : is the distance in hops between the receiver and a cluster head. 


\section{ALGORITHM: HCCD}

1. A cluster head $\left(\mathrm{CH}_{j}\right)$ generates a random number $s_{j}$, i.e., a nonce.

2. $C H_{j}$ sets the field $i=1$.

3. $C H_{j}$ calculates the value $\operatorname{Max}_{j}=h^{t}\left(s_{j}\right)$.

4. $C H_{j}$ generates the $C I D$ message:

$$
<\operatorname{Max}_{j}, h^{i}\left(s_{j}\right), i>\text {. }
$$

5. The receiver node verifies that the sender node is $i$-hops away by applying the following criteria:

- If $\operatorname{Max}_{j}=h^{t-i}\left(h^{i}\left(s_{j}\right)\right)$, then the CID message is valid.

- Else, the receiver node discards the CID message.

6. If the $C I D$ message is valid, then the receiver node generates a new $C I D$ message with the hop count increased by one and applying the hash function to $h^{i}\left(s_{j}\right)$ :

$$
<\operatorname{Max}_{j}, h\left(h^{i}\left(s_{j}\right)\right), i+1>\text {. }
$$

Consider that the hash function $h(x)$ and the value of $t$ are known by all nodes in the network. For our purposes, we suppose that the malicious attacker is not able of generating a valid nonce $s_{j}$. Algorithm HASH-CHAINED_CID_DISSEMINATION (henceforth HCCD for brevity) formalizes our proposal.

Algorithm HCCD works as follows: firstly, a valid cluster head $\left(\mathrm{CH}_{j}\right)$ generates a random number $s_{j}$, i.e., a nonce that is only known by the originator of the message. Then, it initializes the hop count field $i$ equal to one and computes the $\operatorname{Max}_{j}$ value by applying $t$ times the hash function $h(x)$ to the nonce $s_{j}$, such as $M a x_{j}$ is equal to $h^{t}\left(s_{j}\right)$. We assume that $M a x_{j}$ and the value of $t$ are known by all the nodes in the network during the execution of the protocol. Additionally, $C H_{j}$ applies $i$ times the hash function to $s_{j}$, to obtain $h^{i}\left(s_{j}\right)$. Then, $C H_{j}$ generates a $C I D$ message with the fields: $\left\langle\operatorname{Max}_{j}, h^{i}\left(s_{j}\right), i\right\rangle$. The receiver node verifies that the CID message is valid by applying $t-i$ times the hash 
function to $h^{i}\left(s_{j}\right)$ and comparing the result with $\operatorname{Max}_{j}$. Therefore, if $\operatorname{Max}_{j}$ is equal to $h^{t-i}\left(h^{i}\left(s_{j}\right)\right)$, then the hop count value $i$ has not been altered and the received CID message is valid. Finally, the receiver node joins $\mathrm{CH}_{j}$ until it receives a CID message from a different cluster head with a lower hop count value. In the mean time, the receiver node generates periodically CID messages announcing its cluster head and the hop count distance to reach it, i.e., $<\operatorname{Max}_{j}, h\left(h^{i}\left(s_{j}\right)\right), i+1>$.

Theorem 1 Given a HOLSR network applying the algorithm HCCD for the dissemination of CID messages, such that malicious nodes in the network are not able of generating a valid nonce $s, h$ is a strong one-way hash function, $i$ is the distance in hops to reach a cluster head $j$ and $M a x_{j}$ is a value obtained by applying $t$ times $h$ to the nonce $s_{j}$. Then algorithm HCCD guarantees that a malicious node cannot generate a valid CID message with a hop count value $k \neq i$, such that $\operatorname{Max}_{j}=h^{t-k}\left(h^{i}\left(s_{j}\right)\right)$.

Proof According to algorithm HCCD , $\operatorname{Max}_{j}=h^{t}\left(s_{j}\right)$ and a CID message is valid if $\operatorname{Max}_{j}=h^{t-i}\left(h^{i}\left(s_{j}\right)\right)$. Then, let us assume that there exists a value $k \neq i$ such that $\operatorname{Max}_{j}=h^{t-k}\left(h^{i}\left(s_{j}\right)\right)$. Thus, $h^{t}\left(s_{j}\right)=h^{t-k}\left(h^{i}\left(s_{j}\right)\right)$. Then, function $h$ does not meet the weak collision resistance property of strong one-way hash functions due to $h^{t-k}\left(h^{i}\left(s_{j}\right)\right)$ and $h^{t-i}\left(h^{i}\left(s_{j}\right)\right)$ are both equal to $\operatorname{Max}_{j}$. Therefore, $h^{t}\left(s_{j}\right)$ is equal to $h^{t-k}\left(h^{i}\left(s_{j}\right)\right)$ only if $k$ is equal to $i$.

\subsubsection{Wormhole Attack in HOLSR}

In Section 6.6.1, we presented a malicious node that might alter the hop count field to unsettle the cluster formation process. However, other variations of the attack can be perpetrated. Consider an adversary with more than one interface. A malicious node may create an invalid cluster by tunneling valid CID messages from a cluster in a different level. This is a variation of a wormhole attack.

In Fig. 6.10, node $M_{1}$ never reports that it has two interfaces and creates a tunnel from cluster C1.B1 to cluster C2.A2. The malicious node $M 1$ receives valid CID messages 


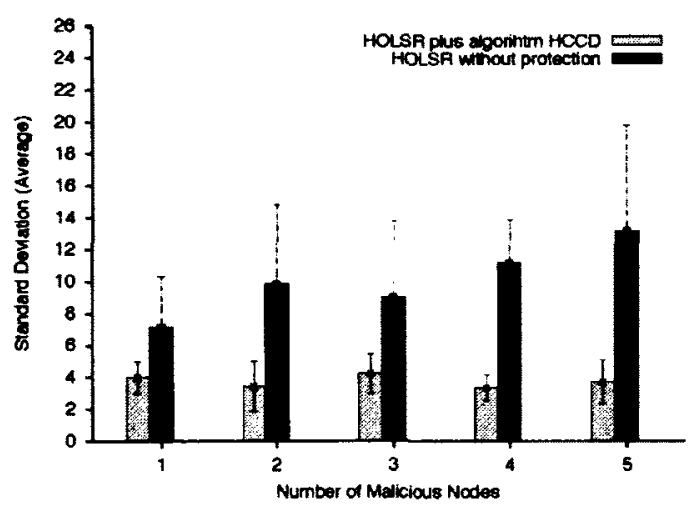

(a) Test $1(\sigma \leq 5)$.

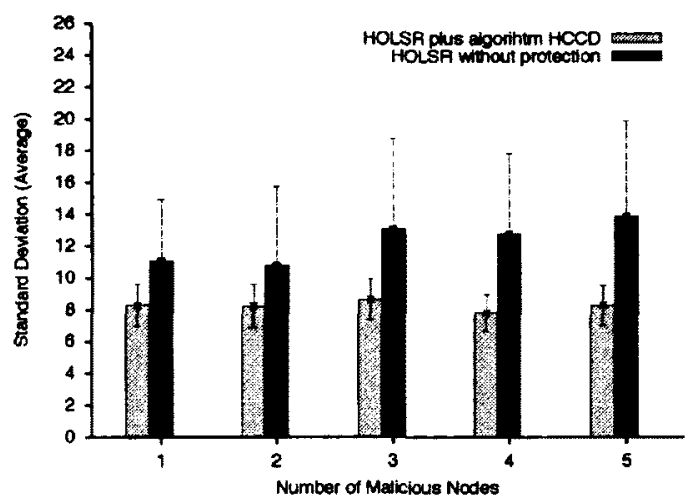

(c) Test $3(7<\sigma \leq 10)$.

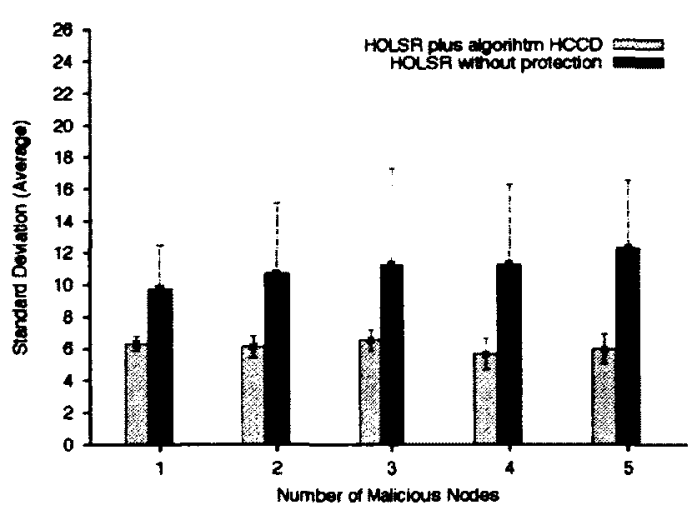

(b) Test $2(5<\sigma \leq 7)$.

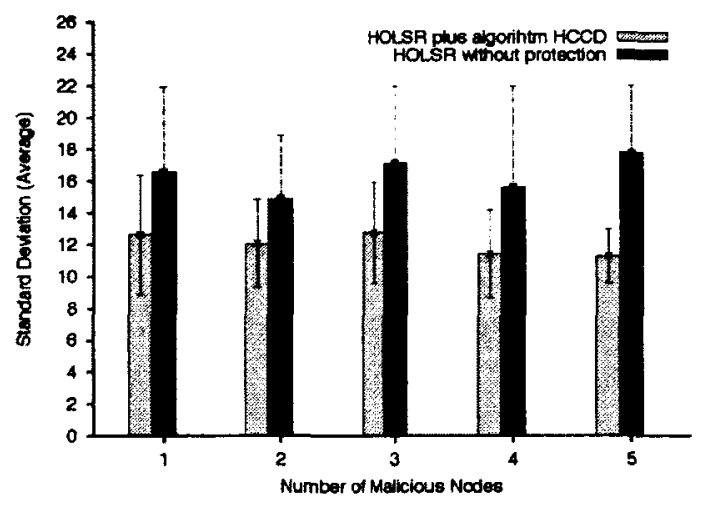

(d) Test $4(10<\sigma)$.

Figure 6.9: Standard deviation of the average number of nodes per cluster testing different HOLSR networks with up to five malicious nodes and applying algorithm HCCD .

from cluster head $C 1$ and retransmit them to cluster C2.A2. The malicious node can not impersonate a cluster head. Hence, if the hop count value is equal to one (i.e., it received the message from a valid cluster head), then it may increase it following the rules of the protocol to avoid being detected. As a consequence, the receivers at level two may join incorrectly a cluster head from level one, creating an invalid cluster. Affected nodes reject valid control traffic information from other nodes at the same level. Additionally, the communication between cluster heads may be disrupted and the propagation of HTC messages is affected. To avoid this attack the algorithm HCCD needs to be modified. When a cluster head generates a CID message, the $\operatorname{Max}_{h}$ value must include an identifier of the interface that it is using to generate the messages. Then, algorithm $\mathrm{HCCD}_{2}$ is proposed. $\mathrm{HCCD}_{2}$ must consider:

- $l_{i f}$ identifies the interface (i.e., level) through which the message has been generated. 


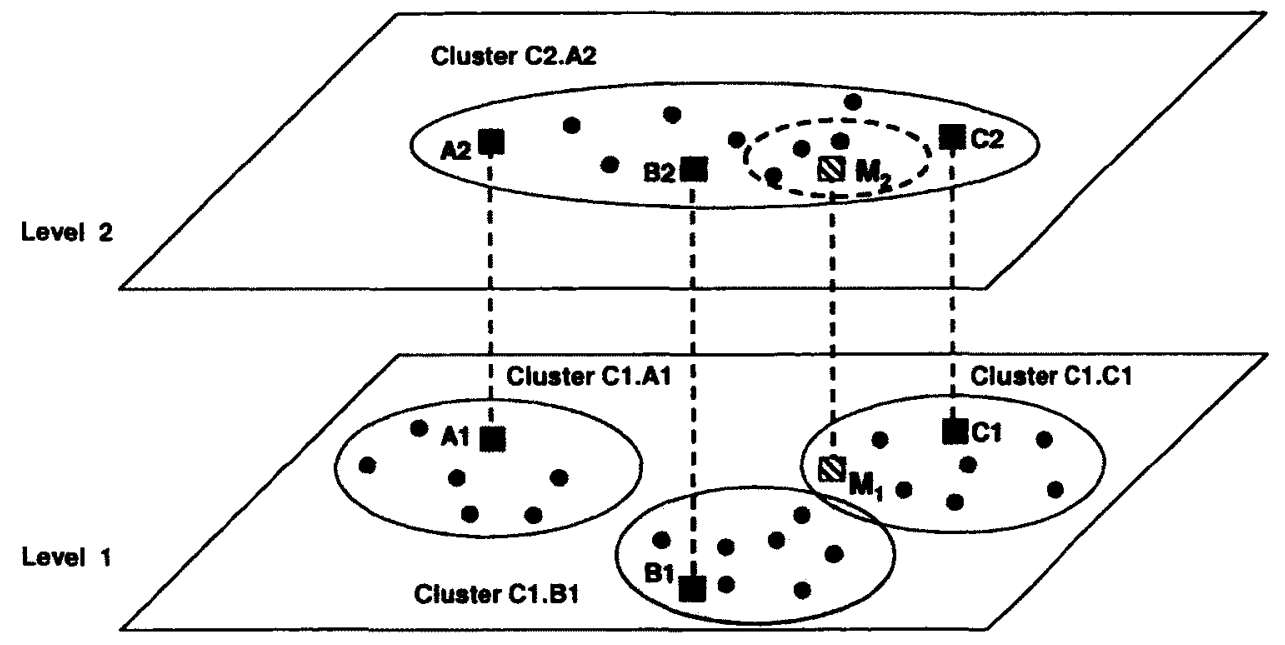

Figure 6.10: Wormhole attack in HOLSR.

This value is known and used by every node with the same interface to communicate.

- $C H_{j}$ calculates the value $\operatorname{Max}_{h}$ including $l_{i f}$ every time function $h(x)$ is applied, i.e., $t$ times. Then, $\operatorname{Max}_{h}=h^{t}\left(\left(\left(s_{j}, l_{i f}\right), l_{i f}\right), \ldots, l_{i f}\right)$

Theorem 2 Given a HOLSR network applying the algorithm $\mathrm{HCCD}_{2}$ for the dissemination of CID messages, such that malicious nodes in the network are not able of generating $a$ valid nonce $s, h$ is a strong one-way hash function, $i$ is the distance in hops to reach a cluster head $j, l_{i f}$ identifies the interface of $\mathrm{CH}_{j}$ through which a CID message is generated and $\mathrm{Max}_{j}$ is a value obtained by applying $t$ times $h$ to the nonce $s_{j}$ and $l_{i f}$. Then algorithm $\mathrm{HCCD}_{2}$ guarantees that a malicious node cannot use a valid CID message from level $l_{i f}$ to level $l_{i f}^{\prime}, l_{i f}^{\prime} \neq l_{i f}$ such that $\operatorname{Max}_{h}==h^{t-i}\left(h^{i}\left(x_{i}\right), l_{i f}^{\prime}\right)$

Proof According to algorithm $\mathrm{HCCD}_{2}, \operatorname{Max}_{h}=h^{t}\left(x_{t}\right)$ and a CID message is valid if $\operatorname{Max}_{h}==h^{t-i}\left(h^{i}\left(x_{i}\right), l_{i f}\right)$. Then, let us assume that there exists a value $l_{i f}^{\prime} \neq l_{i f}$ such that $\operatorname{Max}_{h}==h^{t-i}\left(h^{i}\left(x_{i}\right), l_{i f}^{\prime}\right)$. Then, function $h$ does not meet the weak collision resistance property of strong one-way hash functions due to $h^{t-i}\left(h^{i}\left(x_{i}\right), l_{i f}\right)$ and $h^{t-i}\left(h^{i}\left(x_{i}\right), l_{i f}^{\prime}\right)$ are both equal to $\operatorname{Max}_{j}$. Therefore, $h^{t-i}\left(h^{i}\left(x_{i}\right), l_{i f}^{\prime}\right)$ is equal to $\operatorname{Max}_{h}$ only if $l_{i f}$ is equal to $l_{i f}^{\prime}$. 
ALGORITHM: $\mathrm{HCCD}_{2}$

1. A cluster head $\left(\mathrm{CH}_{j}\right)$ generates a random number $s_{j}$, i.e., a nonce.

2. $C H_{j}$ sets the field $i=1$.

3. $C H_{j}$ calculates the value $\operatorname{Max}_{h}=h^{t}\left(x_{t}\right), h^{t}\left(x_{t}\right)$ is equal to:

- $h^{t}\left(x_{t}\right)=h^{t-1}\left(h^{t-1}\left(x_{t-1}\right), l_{i f}\right)$.

- $h^{1}\left(x_{1}\right)=h\left(s_{j}, l_{i f}\right)$.

4. Then, $\mathrm{CH}_{j}$ generates a $C I D$ message with the fields:

$<\operatorname{Max}_{h}, h^{i}\left(s_{j}, l_{i f}\right), i, l_{i f}>$.

5. The receiver node verifies the interface through which the message has been generated and that the sender node is $i$-hops away by applying the following criteria:

- If $\operatorname{Max}_{h}==h^{t-i}\left(h^{i}\left(x_{i}\right), l_{i f}\right)$ then the $C I D$ message is valid.

- Else, the receiver node discards the $C I D$ message.

6. If the $C I D$ message is valid, the receiver node generates a new $C I D$ message with the hop count increased by one and applying the hash function $h$ to $h^{i}\left(x_{i}\right)$ and $l_{i f}$ :

$<\operatorname{Max}_{h}, h\left(h^{i}\left(x_{i}\right), l_{i f}\right), l_{i f}, i+1>$.

\subsection{Results and Simulations Setup}

In this section, we describe the experiments and results after assessing the effectiveness of our proposed countermeasure to the cluster formation attack presented in Section 6.6. We conducted our experiments using the NS-3 simulator [40], version 3.9. We modified the original OLSR code developed by Ros and Carneiro to implement the hierarchical approach (i.e., HOLSR), attack, and countermeasure described in Section 6.6.2. We tested our algorithm in an HOLSR network with two levels, 200 nodes in the first level with only one interface and four nodes with up to two interfaces (i.e., cluster heads). The transmission range for nodes in the first level and second level is $120 \mathrm{~m}$ and $500 \mathrm{~m}$ respectively. Nodes at the first level are placed following a uniform distribution in an area of $1000 \mathrm{~m}$ 
by $1000 \mathrm{~m}$. We assume that the administrator of the network can decide the best criteria to distribute the cluster heads. Thus, we divide our scenario in four quadrants and place a cluster head in the center of each of them. We also assume that the malicious node knows the position of the cluster heads and sets itself in the border of two different clusters to maximize the impact of an attack. We also assume that the malicious nodes do not collude to perform an attack, no data traffic is generated and all the scenarios are static.

In an ideal scenario, the number of nodes per cluster must be equally balanced. However, due to the position of the nodes in the network this is not always possible. Additionally, the presence of misbehaving nodes may disproportionately increase the imbalance of the number of nodes per cluster. We compute the average of the standard deviation of the number of nodes per cluster with up to five malicious nodes launching the cluster formation attack. Then, we compare the average of the standard deviation of the number of nodes per cluster on a series of simulated HOLSR networks without protection and the average of the standard deviation of the number of nodes per cluster but applying algorithm HCCD. We use the standard deviation $(\sigma)$ as a measure of dispersion. The standard deviation is computed with the formula: $\sigma=\sqrt{\frac{\sum\left(x_{j}-\bar{X}\right)^{2}}{N_{C H}}}$ and expressed in the same units as the data, where $x_{j}$ is equal to the number of nodes in the cluster $j, \bar{X}$ is the average of nodes per cluster, i.e., total number of nodes $(n)$ over the number of clusters $\left(N_{C H}\right)$. In our experiments, the standard deviation formula can be simplified as follows: $\sigma=\sqrt{\frac{\sum\left(x_{i}-50\right)^{2}}{4}}$. Fig. 6.9 shows how the CID attack affects the average of the standard deviation of 100 experiments with different topologies and $90 \%$ confidence interval.

To present our results, we consider two factors that affect the distribution of nodes per cluster: the network topology and the presence of malicious nodes. Thus, Fig. 6.9(a) shows the experiments where $\sigma \leq 5$, this means that the distribution of nodes per cluster in the network is relatively balanced when there is no malicious nodes. Fig. 6.9(b) shows the experiments where the distribution of nodes per cluster is less balanced due to the network topology, i.e., $5<\sigma \leq 7$, Fig. 6.9(c) shows the experiments where $7<\sigma \leq 10$, and Fig. 6.9(d) shows the experiments where $10<\sigma$. In each case, the first column 
shows the average of the standard deviation with malicious nodes and implementing algorithm HCCD. Therefore, the distribution of the nodes per cluster is affected only by the network topology. The second column represents the average of the standard deviation with malicious nodes but without applying our algorithm. Notice that the average of the standard deviation and size of the confidence interval increase because the number of nodes per cluster is less balanced due to the network topology plus the effect of the attack.

\subsection{Related Work}

In this chapter, we reviewed the cluster formation phase in HOLSR networks, however other hierarchical approaches based on the OLSR protocol are also vulnerable during the cluster formation stage. Therefore, the hierarchical approaches presented in Section 6.3 i.e., the cluster OLSR (C-OLSR) [88] proposed by Ros et al., the Multi-level OLSR Routing using the Host and Network Association messages Extension (MORHE) [97] presented by Voorhean et al. and the tree-based logical topology $[17,16]$ to provide hierarchical routing presented by Baccelli., are are also affected by the cluster formation attacks presented in this chapter.

In [44], Hong et al., present a solution to secure OLSR (SOLSR). Authors present a wormhole detective mechanism and authentication to strengthen the neighbor relationship establishment. Thus, they use digital signature to ensure the non-mutable fields and hash chains to secure Hop Count and TTL fields. Their solution is similar to our proposed algorithm, however it is implemented in flat OLSR to protect only standard control traffic messages. Kush and Hwang, present in [56] a mechanism based in hash chains to secure AODV. Then Hashing is done for route request and reply messages to achieve complete security in terms of availability, integrity and authentication, minimal overhead, network performance in terms of throughput and node mobility. Similarly, Hu et al., [45] propose a hashing mechanism to secure distance vector routing protocols.

A hierarchical approach similar to HOLSR which uses HNA messages instead of HTC messages for inter-cluster communication is proposed by Arce et al. in [15]. Like 
HOLSR, cluster heads are predefined then is not necessary a cluster head selection algorithm, however a cluster formation mechanism is needed. Therefore, any strategy that uses the distance in hops as the main parameter to invite other nodes to join a particular cluster head will be affected by the attack presented in this chapter.

Clustering is commonly used to reduce the amount of control traffic information and to improve efficiency and scalability. However, in [43], authors propose a clustering mechanism to manage and to distribute cryptographic keys in an OLSR-based network. Hajami et al., propose an improved model for ad hoc clustering based on multi hops and network density. To calculate the density of a node the algorithm uses the information contained in the Hello messages, thus, it is not necessary to implement new kinds of messages. The goal is to select a reduced number of stable cluster heads that will be employed for keys exchange.

In [60], Liu et al., propose a hierarchical routing scheme for MANETs to preserve privacy and anonymity. Authors consider that if the number of nodes in a MANET with cryptographic capabilities scales up, then the number of public key operations increases generating additional overhead that affects the routing and data communication performance. Thus, authors propose a Hierarchical Anonymous On-demand Routing Protocol (HANOR) to achieve an efficient anonymous protocol suitable for hierarchical architectures. HANOR provides two levels of anonymity: intra-group and inter-group anonymous routing. Nevertheless, the authors do not consider in their proposal malicious attacks against service availability or defense mechanisms in a network with compromised nodes.

\subsection{Conclusion}

In this chapter, we reviewed security issues in hierarchical OLSR-based networks. HOLSR has been designed to improve scalability in MANETs. However, the protocol has been designed without security countermeasures. We presented the effect of flooding disruption attacks that affect the topology map acquisition HOLSR. These kind of attacks affect either the MPR selection process or the flooding of control traffic information for inter-cluster or intra-cluster communication. We presented a set of strategies to mitigate the effect of 
this kind of attacks. According to our experiments, it is possible to mitigate the effect of flooding disruption attacks by selecting the MPR sets with additional coverage or generating control traffic with redundant information.

Additionally, we proposed a method to protect the cluster formation stage in HOLSR. Our mechanism prevents an attacker from maliciously altering the hop count field in CID messages. Thus, we present an algorithm based on hash chains that allows to detect and discard invalid CID messages. Our experiments show that the distribution of nodes is less balanced when the hop count in CID messages is maliciously altered. We also show that we can prevent this kind of attacks by applying our proposed algorithm. Notice that our mechanism, can be also applied in other hierarchical routing protocols for MANETs that utilize mutable information such as the hop count or TTL fields to organize the network in clusters. 


\section{Chapter 7}

\section{Security Issues in Multipath OLSR}

\subsection{Introduction}

The design of an efficient routing protocol in Mobile Ad-Hoc Networks (MANETs) has become a challenging problem. This kind of networks are more prone to both link and node failures due to restricted energy or mobility. Additionally, if a node misbehaves during the execution of the routing protocol the connectivity of the network is compromised. Multipath routing has been proposed in MANETS to improve scalability, fault tolerance, security, load-balancing, energy-conservation and Quality-of-Service (QoS) [70]. Unlike the single path strategy, in a multipath approach different paths are computed between a source and a destination to increase the routing resilience against failures. According to Tarique et al. [92], in multipath routing protocols there are three major challenges to be addressed: a) discovery multiple routes, i.e., strictly disjoint routes or routes with nodes or links in common, b) path selection, i.e., multiple paths can be used as backups or simultaneously for parallel data transmission, and c) load distribution, i.e., how data is transmitted trough the multiple routes. In this thesis, we address security issues that affect either the discovery or selection of routes in link-state multipath routing protocols.

Several OLSR-based multipath routing strategies have been proposed. In general, OLSR-based multipath protocols have two phases: Topology Discovery and Route Computation. In the first phase, the nodes obtain information about the network topology through the exchange of Hello and TC messages. In the second phase, the nodes compute multiple paths to a particular destination in the network based on the information gathered during the first phase. Ideally, to increase the resilience against failures or to cope with security threats, a node may construct strictly disjoint paths, i.e., none of the computed routes share links or nodes. 
The optimizations in OLSR reduce the chances of constructing strictly disjoint paths. In the first optimization, TC messages are generated exclusively by the MPRs. In the second optimization, the MPRs report only their selector set. Additionally, the original algorithm defined in RFC3626 [34] to compute MPR sets minimizes the number of nodes selected as MPRs to reduce the overhead generated by control traffic messages. Thus, only a subset of nodes generate partial link-state information. Hence, some important links to the construction of strictly disjoint paths are unannounced.

The flooding of link-state information is also affected by misbehaving nodes in the network. In [24], we present a taxonomy of flooding disruption attacks that affect either the flooding of control traffic information or the selection of the MPRs in OLSR-based networks. All the multipath routing strategies based on the selection of MPRs as a flooding mechanism are susceptible to these attacks. The attacks have impact either in the topology discovery or route computation phases. In [102, 103, 104, 105], Yi et al., proposed a multipath extension to OLSR, Multipath OLSR (MP-OLSR). Nevertheless, as many other routing protocols based on OLSR, MP-OLSR has been proposed without security measures. We selected it as an example to present drawbacks and security risks in multipath routing protocols based on OLSR. MP-OLSR is a hybrid multipath routing protocol with multiple description coding for data transfer. In MP-OLSR, the construction of multiple paths leverages on Dijkstra's algorithm to find optimal routes in terms of hops. MP-OLSR uses TC messages with redundant information to increase the chances of constructing disjoint routes. MP-OLSR comprises the TC redundancy (TCR) parameter defined in RFC3626 [34] to include more information in every TC message. This parameter is defined in the RFC3626 [34] and has three possible values:

- If TCR is equal to zero, then MPRs report its selector set.

- If TCR is equal to one, then MPRs report its selector table and its MPR set.

- If TCR is equal to two, then MPRs report its one-hop neighbor set.

This information allows every node to construct and to maintain its topology table [47]. 
However, in some cases, TC messages with redundant information are not enough. MPOLSR does not considers nodes with partial views of the network nor flooding disruption attacks. Additionally, the computed routes are not necessarily disjoint. The algorithm computes several routes but it is impossible to know how many of them are strictly disjoint.

To address these problems, we propose to compute MPR sets with additional coverage during the network topology discovery phase and a mechanism to obtain, if possible, $t+1$ disjoint paths during the route computation phase, where $t$ is a positive integer. Additional coverage in the selection of the MPRs is defined in [34], as the ability of a node to select redundant MPRs to advertise its presence to more nodes in the network. In this manner, extra coverage helps to maintain the integrity of the network in spite of the presence of misbehaving nodes during the network topology map acquisition. The selection of MPRs with extra coverage is defined in RFC3626 [34]. We named this approach a $k$-CoveredMPR selection. However, the overhead due to the excessive number of TC messages reduces the performance of the network. This problem is addressed by the $k$-Robust-MPR selection presented in [23], which balances security and traffic overhead. In OLSR, the MPRs form a Connected Dominating Set (CDS). A CDS is a subset of connected nodes such that if a node in the network is not part of the CDS, then it has a link to a node in the CDS. We define an MPRCDS as a CDS such that every node in the CDS has been selected as an MPR. When the nodes select their MPRs following a $k$-Covered-MPR selection we obtain a $k$-CCDS. When the nodes compute their MPRs following a $k$-Robust-MPR selection we obtain a $k$-RCDS. These variation on the selection of MPRCDS are formally defined in Section 7.5.1. We propose the function DM-OLSR to construct multiple strictly disjoint paths. Our improved mechanism, utilizes additional coverage in the selection of MPRs during the topology discovery phase.

\subsubsection{Contributions}

In this Chapter, we present the Disjoint Multipath OLSR (DM-OLSR) function to address the following problems: i) a partial view of the network topology, ii) flooding disruption attacks, and iii) load balancing in OLSR-based networks. DM-OLSR, the nodes select 
their MPRs with additional coverage during the topology discovery phase and compute, when possible, $t+1$ strictly disjoint paths during the route computation phase. Our mechanism privileges the nodes with the smallest number of nodes in their selector set to be included in the computed paths. Clearly, in sparse networks it is not always possible to compute disjoint paths. Nevertheless, multipath routing takes advantage of large and dense networks. Then, we focus on the cases where the construction of multiple disjoint paths is affected either by an incomplete view of the network topology or by the presence of a misbehaving node that perpetrates a flooding disruption attack.

\subsection{Related Work}

In this section, we present other multipath routing strategies proposed for MANETs. Multipath routing in MANETs has been widely studied in reactive routing protocols. In [92], Tarique et.al., present a review of multipath routing protocols for mobile ad hoc networks. The strategies presented are derived from the Dynamic Source Routing(DSR) [50] and the Ad hoc On-demand Distance Vector (AODV) [79] routing protocols. The authors classify several multipath routing protocols into different categories based on their main goals (e.g., reliability, QoS, etc.). A similar review of issues and challenges in multipath routing based on reactive approaches is presented in [70] by Mueller et al. In [81] proposed a Multi-Path

Routing Protocol with Load Balance (MRP-LB) to reduce congestion in MANETs. The protocol is based on the reactive approach. The main objective of MRP-LB is to split data traffic simultaneously and equally to multiple disjoint paths. The protocol considers a route discovery and route maintenance phases. In addition, it also defines two more phases: data transmission and load balance maintenance.

In [82], Pham and Perreau analyzed and compared the reactive single-path and multipath routing with load balance mechanisms in MANETs, in terms of overhead, traffic distribution and connection throughput. Their results showed multi-path routing mechanism creates more overheads but provides better performance in congested networks. Nevertheless, reactive approaches are based on the generation and exchange of route request (RRQ) and route replay (RRP) messages. According to Yi et al. [102], multipath reactive routing 
protocols increase the number of route request messages. When intermediate nodes process duplicate request messages. As a result, redundant overhead packets are introduced in the network. Additionally, to find node-disjoint or link-disjoint paths, some multipath routing protocols prevent an intermediate node from sending reply messages from its route cache. Therefore, a source node has to wait until a destination replies. Hence, the route discovery process of a multipath routing protocol takes longer compared to that of DSR or AODV protocols.

Moreover, several multipath routing approaches take advantage of the proactive behavior and the MPR flooding mechanism proposed in OLSR. In [55], Kun et al., proposed a different version of multipath OLSR using IP-source routing. Based on Dijkstra's algorithm, nodes calculate multiple disjoint paths. Additionally, the authors introduce an algorithm of load-assigned to transmit data through the paths based on the congestion information of all intermediate nodes on each path. Badis and Al Agha [18], also proposed a path selection criteria and multi-path calculation based on bandwidth and delay to improve QoS in OLSR-based networks (QOLSR). The resulting protocol, computes multiple loopfree and node-disjoint paths. The authors implement the shortest-widest path algorithm to guarantee loop-free routes. Additionally, they evaluated and compared QOLSR multipath routing versus a QOLSR single-path routing using a scalable simulation model.

In [14], Aiache et al., proposed an strategy to improve security and performance of an ad hoc network through a multipath routing strategy. Frequently, when security increases, the QoS decreases. The authors proposed a solution that provides anonymity and security to ad hoc networks with a limited impact on QoS. The authors also give some security proofs of their solution for ad hoc networks. In [90], Srinivas and Modiano proposed algorithms for finding minimum energy disjoint paths in wireless networks. Their main contribution is a polynomial time algorithm for the minimum energy $k$ node-disjoint problem. Node-disjoint paths are more resilient to failures. However, the authors showed that link-disjoint paths save more energy.

Conti et al. provided in [61] a method to improve the performance and reliability 
of nodes communication in presence of misbehaving nodes. The authors proposed and evaluated a forwarding policy that is based on multi-path routing and considers nodes reliability and routes length in forwarding decisions. The authors showed how their proposed mechanism improves the reliability of TCP data transfers. In particular, they showed that the simultaneous use of multiple paths offers higher throughput and continuous network connectivity when compared to single path forwarding. The authors evaluated both fault conditions and intentional nodes misbehavior in MP-OLSR networks. Zhou et al. proposed in [106] the Source Routing based Multi-Path OLSR (SR-MPOLSR) protocol. The protocol implements Dijkstra's algorithm to calculate multiple disjoint routes and to allocate the loads in a weighted round-robin fashion. Data transmission at the source is carried out through predetermined multiple paths (i.e., source routing).

All strategies proposed, are not analyzed from a security perspective. Both reactive and proactive approaches are vulnerable to misbehaving nodes that interrupt the flooding of control traffic information. Specifically with OLSR-based multipath routing protocols, all approaches are affected by an incomplete view of the network topology. These problems are addressed in the following sections.

\subsection{Multipath OLSR}

In this section, we describe in detail the Multipath OLSR (MP-OLSR) routing protocol proposed by $\mathrm{Yi}$ et al. in [103, 104, 105, 102]. MP-OLSR is proposed to enhance load-balancing, energy-conservation, QoS and security. MP-OLSR is a hybrid multipath routing protocol that takes advantage of the MPR mechanism to flood the network with control traffic information. In MP-OLSR, the OLSR proactive behavior is changed for ondemand route computation. MP-OLSR becomes a source routing protocol. There are two phases: topology discovery and routes computation. During the topology discovery phase, nodes obtain a partial topology map just like in OLSR. However, MP-OLSR nodes do not construct routing tables. During the routes computation phase, nodes calculate multiple paths to reach any other node in the network following an on-demand scheme. MP-OLSR implements Multiple Description Coding (MDC) for data transfer. MDC adds redundancy 
to information streams and split them up into several sub-streams to improve the integrity of data. These sub-streams are sent along multiple paths from the source to the destination. MP-OLSR implements source routing with route recovery and loop detection to adapt to the changes in the network topology. Thus, when data transfer is required, route recovery and loop detection allow every node to detect if a path is not valid anymore and to find a new path to reach the final destination. MP-OLSR implements the MultiPath Dijkstra's algorithm to discover the shortest routes. The paths that are obtained can be grouped in two categories:

1. Disjoint: In this category we have two types of disjoint paths: node-disjoint and link-disjoint. Node-disjoint paths type do not share nodes except for the source and destination nodes. Link-disjoint paths can share some nodes but all the links are different.

2. Inter-twisted: In this case, the paths may share several links.

To construct disjoint paths, MP-OLSR defines cost functions to obtain new paths that tend to be node-disjoint or link-disjoint. Once a path is computed, a function $f_{p}$ is used to increase the costs $c$ of the links that belong to the computed path, e.g., $f_{p}(c)=3 c$. A function $f_{e}$ is defined to increase the cost of the links of the nodes included in the path previously obtained. In MP-OLSR, neither nodes nor links used in computed paths are eliminated. This strategy allows MP-OLSR to construct multiple paths in sparse networks where is not always possible to find strictly node-disjoint paths. In addition, to increase the chances of constructing node-disjoint paths, the MPRs report all their one-hop neighbors (i.e., the TCR parameter is equal to two). Therefore, to construct disjoint paths, there are three possibilities:

- if $f_{i d}=f_{e}<f_{p}$, then paths tend to be link-disjoint.

- if $f_{i d}<f_{e}=f_{p}$, then paths tend to be node-disjoint.

- if $f_{i d}<f_{e}<f_{p}$, then paths also tend to be node-disjoint, but when not possible they tend to be link-disjoint. 
For example, in Fig. 7.1(a), node $s$ attempts to construct multiple paths to node $d$. Consider initial cost $c$ of every link equal to one and $f_{p}(c)=3 c$ and $f_{e}(c)=c$, i.e., a penalty is only applied to the used links. The first time the Dijksra's algorithm is applied, the computed path is $s \rightarrow b \rightarrow h \rightarrow d$. The cost of the links $(s, b),(b, h)$ and $(h, d)$ is changed from one to three using $f_{p}$, see Fig. 7.1(b). The path: $s \rightarrow c \rightarrow f \rightarrow i \rightarrow d$, is a node-disjoint path. However, the path $s \rightarrow a \rightarrow b \rightarrow i \rightarrow d$ has the same chances of being discovered. This path is link-disjoint. If that path is selected, then is not possible to obtain node-disjoint routes. The path: $s \rightarrow c \rightarrow f \rightarrow h \rightarrow j \rightarrow d$, is also a link-disjoint path. The cost of all used links is set to three, see Fig. 7.1(c). To obtain paths that tend to be node-disjoint, we define functions $f_{p}(c)=3 c$ and $f_{e}(c)=2 c$. In this case, the penalty is also applied to the used nodes. First, the path $s \rightarrow b \rightarrow h \rightarrow d$ is computed and the cost of the links is updated. The links that include a node in the computed path -except for the source $s$ and the destination $d$ - are set to two, see Fig. 7.1(d). Then, the next paths we can obtain are: $s \rightarrow c \rightarrow f \rightarrow i \rightarrow d$ and $s \rightarrow a \rightarrow e \rightarrow g \rightarrow j \rightarrow d$. These three paths are node-disjoint. The path: $s \rightarrow a \rightarrow b \rightarrow i \rightarrow d$, is an example of an inter-twisted path.

\subsubsection{Route Computation}

The goal of MP-OLSR, is to construct a set $P$ of $t$ paths, with no loops, between a source node $s$ and a destination node $d$. The network topology is represented by a graph $G=$ $(V, E, c)$, where $V$ is the set of vertices $v$ (i.e., nodes), $E \subset V \times V$ is the set of arcs $e$ (i.e., links between nodes) and $c$ a strictly positive cost function. An $\operatorname{arc} e \in E$, is defined as a pair of vertex $\left(v_{q}, v_{q+1}\right)$ with a bidirectional link. A path between a pair of distinct vertices $(s, d)$, is defined as a sequence of vertices $\left(v_{1}, v_{2}, \ldots, v_{m}\right)$ such that $\left(v_{q}, v_{q+1}\right) \in E$, $v_{1}=s$ and $v_{m}=d$. The cost of an arc formed by the vertices $v_{q}$ and $v_{q+1}$ is noted $c\left(e_{q}\right)=$ $\mathrm{c}\left(v_{q}, v_{q+1}\right)$ and is always positive. Additionally, we assume the following considerations:

- The graph $G$ is bidirectional, i.e., $\left(v_{q}, v_{q+1}\right) \in E \Rightarrow\left(v_{q+1}, v_{q}\right) \in E, v_{q} \in V$ and $c\left(v_{q}, v_{q+1}\right)=c\left(v_{q+1}, v_{q}\right)$.

- The graph is loop free, i.e., no arcs join a node to itself.

- Every pair of vertices is not connected by more than one link. 


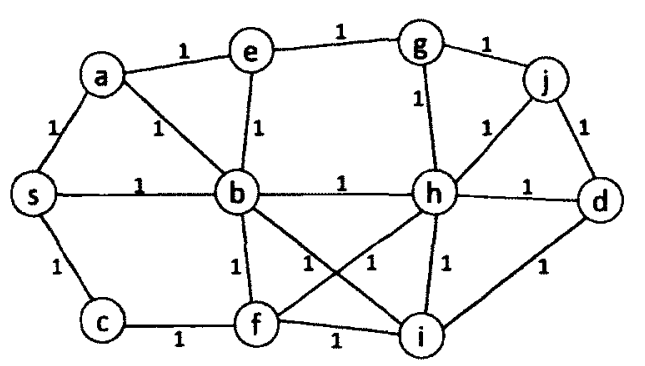

(a) Initial state. The cost of the links is equal to one.

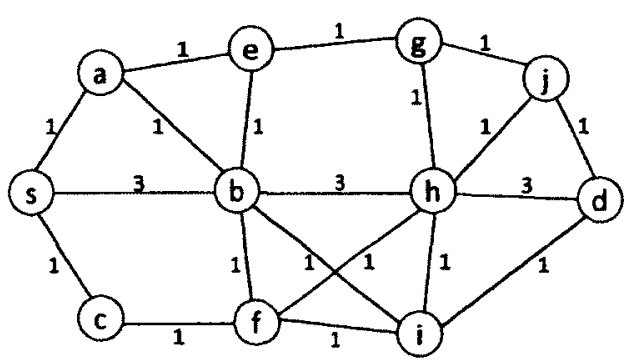

(b) Applying function $f(c)=3 c$ after computing the first path.

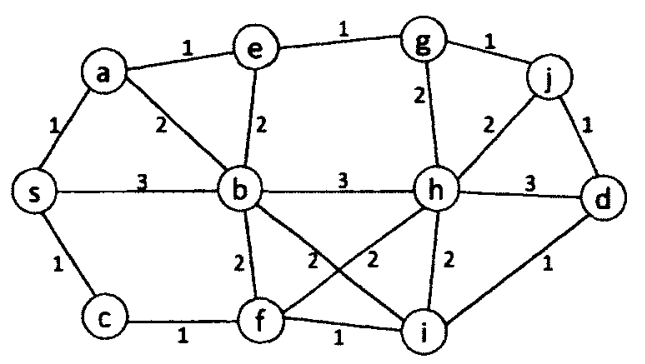

(d) Applying function $f(c)=3 c$ and $f(c)=2 c$ after computing the first path. (c) Applying function $f(c)=3 c$ after computing the second and third link-disjoint paths.

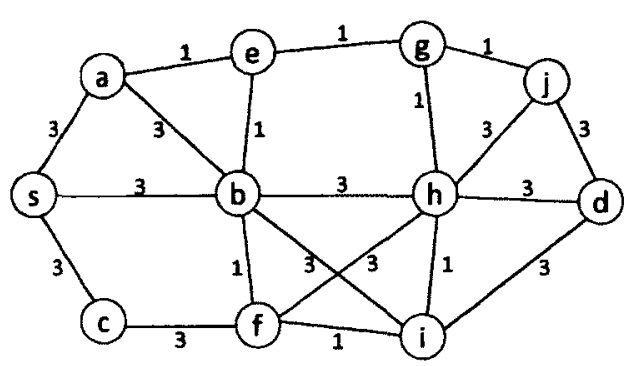

Figure 7.1: Example of constructing multiple paths in MP-OLSR.

MP-OLSR uses the hop count as the metric to select multiple paths, however, other metrics can be used to select the best path, e.g., bandwidth, delay, etc. Given a source node $s$, MP-OLSR will keep an updated flag (updatedFlag) to identify if the routes are still valid. Initially, the updatedFlag is set to false, this means that the routes are not calculated yet or need to be renewed. When a node $s$ needs to calculate a route to node $d$ it verifies the updatedFlag, then:

- If updatedFlag equals false, then node $s$ executes function MultipathDijsktra to compute $t$ routes. After, it saves them in its multipath routing table and sets the parameter updatedFlag equal to true.

- If updatedFlag equals true, then node $s$ obtains a valid route to a node $d$ from its multipath routing table.

When the source node $s$ receives a new Hello or TC message and it detects a change in the network topology, the parameter updatedFlag is set to false. Function MultipathDijsktra obtains a set of $t$ paths $P=\left(P_{1}, P_{2}, \ldots, P_{t}\right)$ from a graph $G=(V, E, c)$. The paths 
in $P$ are not necessarily disjoint, once a path $P_{i}$ is calculated, all the $\operatorname{arcs}\left(v_{q}, v_{q+1}\right) \in E$ such as $v_{q}, v_{q+1} \in P_{i}$ are penalized, i.e., the cost of the links is increased. Function MultipathDijsktra works as follows:

- First, function Dijkstra $(G, s)$ is the standard Dijkstra's algorithm which returns a source tree of the shortest path from node $s$ in graph $G$. Initially, the cost of all the links is set to one.

- Then, function GetPath(SourceTree,d) extracts the path $P_{i}$ from node $s$ to node $d$.

- Given an $\operatorname{arc} e_{q}=\left(v_{q}, v_{q+1}\right)$, function Reverse $\left(e_{q}\right)$ returns the opposite edge $\left(v_{q+1}, v_{q}\right)$. Function $\operatorname{Head}\left(e_{q}\right)$ obtains the vertex edge to $e_{q}$ which $e_{q}$ points, i.e., $v_{q+1}$.

- The procedure is repeated $t$ times until we obtain a set $P$ with $t$ routes to reach node $d$ from a source node $s$

The incremental functions $f_{p}$ is used to increase the cost of the arc $e_{q}$ or Reverse $\left(e_{q}\right)$ that belong to the path $P_{i}$. This will make that future paths tend to be link-disjoint. The incremental function $f_{e}$ is used to increase the cost of the arcs if $H e a d\left(e_{q}\right)$ belongs to $P_{i}$, then this will make that the arcs tend to be node-disjoint. The paths constructed by function MultipathDijsktra do not need to be strictly disjoint. Yi et al. define the minimalcut as the size of the smallest subset of nodes that the source node $s$ can not avoid to reach destination node $d$, i.e., one and two-hop neighbors of nodes $s$ and $d$. For instance, in Fig. 7.1(a), the maximum number of disjoint paths that a source node $s$ can construct is the minimum value between the number of one-hop neighbors (i.e., three) and two-hop neighbors (i.e., four).

\subsection{MP-OLSR Drawbacks and Security Vulnerabilities}

In this section, we review vulnerabilities in multipath routing strategies based on OLSR. In OLSR every node must acquire and maintain a routing table that effectively reflects the 


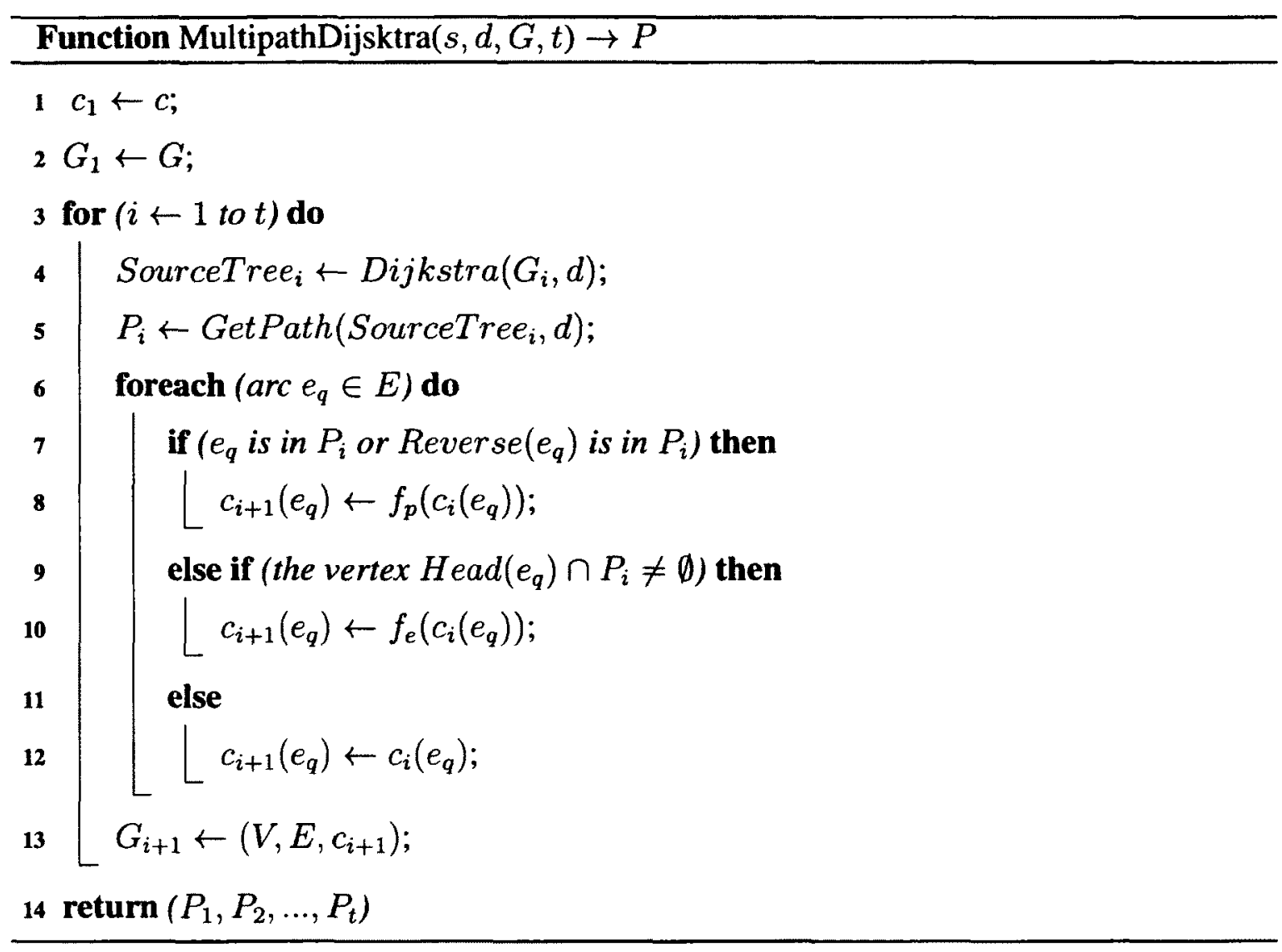

network topology [31]. According to Herberg and Clausen, the routing tables constructed by every node must converge, i.e., all nodes must have an identical topology map. Therefore, the target of a misbehaving node may be that the nodes in the network (a) build inconsistent routing tables that do not reflect the accurate network topology, or (b) acquire an incomplete topology map.

MP-OLSR constructs multiple paths that are not necessarily disjoint. MP-OLSR is affected by the flooding disruption attacks presented in [24]. Thus, an attacker might select an invalid MPR set to prevent other nodes to calculate disjoint paths to reach other nodes in the network. Furthermore, it presents different limitations and vulnerabilities.

1. The nodes in an OLSR system only obtain a partial view of the network topology.

2. OLSR-based networks are vulnerable to flooding disruption attacks, such as the attacks presented in [24]. 


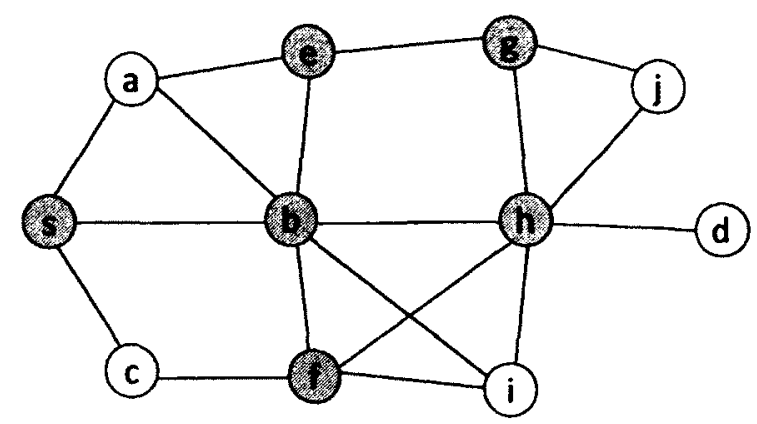

Figure 7.2: Node $s$ perspective of the network topology with parameter TCR equal to two. Gray nodes represent MPRs.

3. The computed shortest path is not always the best option in terms of load balancing or fault tolerance.

Therefore, multipath routing protocols based on OLSR are affected by the vulnerabilities and restrictions that we describe in the following sections.

\subsubsection{Partial Network Topology View}

The Topology Discovery phase in MP-OLSR is based on the exchange of topology control messages. The MPRs generate and forward TC messages to advertise its selector set to other nodes more than two hops away in the network. However, with this information nodes only obtain a partial view of the network topology. This is because TC messages are generated exclusively by the MPRs and the MPRs only report their selector set. In other words, the MPRs only report partial link-state information. Fig. 7.2 shows the network perspective of node $s$ after the topology discovery phase. Notice that node $s$ receives only partial information about the network topology, i.e., the edges $(j, d)$ and $(i, d)$ are never reported in TC messages. These links are not reported because neither $j$ nor $i$ are MPRs. Therefore, from the perspective of node $s$, node $h$ is the only node to reach node $d$ and it is not possible to compute multiple disjoint paths. All possible paths to reach node $d$ are inter-twisted. To increase the chances of finding disjoint paths, the MPRs in MP-OLSR report more information in their TC messages by tunning their TCR parameter. 


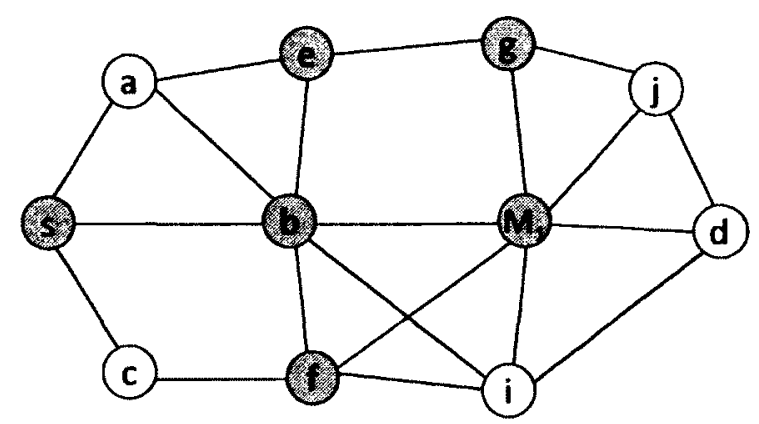

Figure 7.3: Node $M_{1}$ is a misbehaving node.

\begin{tabular}{|c|l|c|l|c|l|l|}
\hline \multirow{2}{*}{ Node } & \multicolumn{2}{|c|}{ 0-Robust-MPR } & \multicolumn{2}{c|}{ 1-Robust-MPR } & \multicolumn{2}{c|}{ 2-Covered-MPR } \\
\cline { 2 - 7 } & Selectors & $S_{d}(v)$ & Selectors & $S_{d}(v)$ & Selectors & $S_{d}(v)$ \\
\hline s & a,c & 2 & a,c & 2 & a,c & 2 \\
a & - & 0 & - & 0 & s,e & 2 \\
b & s,a,e,f,h,i & 6 & s,a,e,f,h,i & 6 & s,a,e,f,h,i & 6 \\
c & - & 0 & - & 0 & s,f & 2 \\
d & - & 0 & - & 0 & i,j & 2 \\
e & a,g & 2 & a,g & 2 & a,b,g & 3 \\
f & b,c,h,i & 4 & b,c,h,i & 4 & b,c,h,i & 4 \\
g & e,j & 2 & e,j & 2 & e,h,j & 3 \\
h & b,d,f,g,i,j & 6 & b,d,f,g,i,j & 6 & b,d,f,g,i,j & 6 \\
i & - & 0 & d & 1 & b,d,f,h & 4 \\
j & - & 0 & d & 1 & d,g & 2 \\
\hline
\end{tabular}

Table 7.1: MPR selectors with or without additional coverage ( $k$ is equal to two).

The TCR parameter is defined locally by every node. Nodes with a different TCR value can coexist in the network. MP-OLSR nodes set their TC parameter equal to two. However, the size of the TC messages increases and in some situations is not enough to report important links. For example, in Fig. 7.2 the edges $(j, d)$ and $(i, d)$ are never reported even if the MPRs set their TCR parameter equal to two. As a consequence, if node $k$ misbehaves, then all the paths to reach node $d$ are compromised.

\subsubsection{Flooding Disruption Attacks in Multipath OLSR}

The flooding mechanism for control traffic information in an OLSR-based network is based on the correct selection of the MPRs. Control traffic messages (i.e., TC, HNA or 


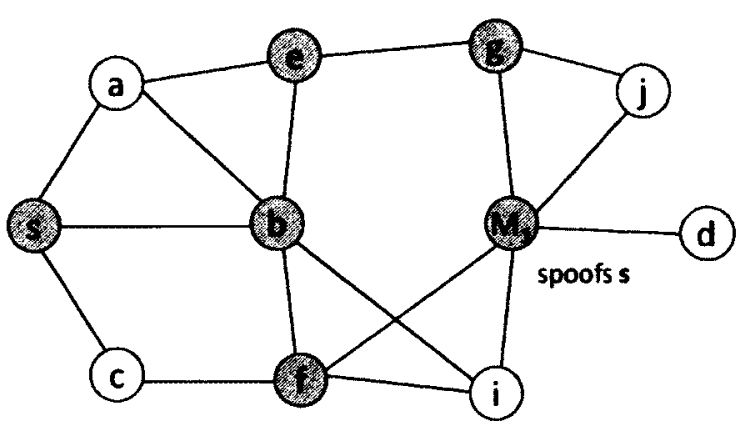

Figure 7.4: Node $s$ perspective of the network topology after an identity spoofing attack. Node $M_{1}$ spoofs the identity of node $s$.

MID messages) are forwarded exclusively by the MPRs. An attacker seeking to interrupt the control traffic flooding can either (a) manipulate the information about the one and twohop neighbors of a given node to cause the MPR selection to fail, or (b) misbehave during the generation and forwarding processes. Flooding disruption attacks can be launched to affect both Topology Discovery and Route Computation phases. For instance, when an attacker interrupts the flooding of topology control messages important links or nodes are lost. Thus, the nodes obtain only a partial view of the network topology and this reduces the chances of constructing multiple strictly disjoint paths to a receiver node. A detailed description of the attacks is presented in the following sections.

\section{Incorrect MPR Selection}

A misbehaving node can affect the MPR selection process by injecting incorrect information. Consider $M_{1}$ in Fig. 7.3 as a misbehaving node. Node $M_{1}$ may launch the following attacks:

Identity Spoofing. The identity spoofing attack [31] is performed by a malicious node pretending to be a different node in the network. The goal of the attack is to report false information about nodes one or two-hops away in order to maliciously affect the MPR selection process. Fig. 7.4 illustrates an example where node $M_{1}$ spoofs the identity of node $s$ and broadcasts hello message advertising valid links with nodes $g, j, d, i$ and $f$. As the information extracted from different Hello messages is accumulative. Node $b$ selects incorrectly only $s$ as the only element in its MPR set. The links $\left(b, M_{1}\right)$ and $\left(M_{1}, \mathrm{~d}\right)$ are 
reported only if $M_{1}$ generates TC messages with its real identity. As a consequence, from the perspective of node $s, d$ is isolated and reachable only trough $M_{1}$. In order to maximize the impact of the attack, a misbehaving node may simultaneously spoof multiple identities by overhearing TC and Hello messages for a while.

Link Spoofing. The link spoofing attack [31] is performed by a malicious node that reports an inexistent link to other nodes in the network. The objective of the attacker is to manipulate the information about the nodes one or two hops away and be selected as part of the MPR set. Once the malicious node has been selected as an MPR, it neither generates nor forwards control traffic information. The flooding disruption attack due to link spoofing is illustrated in Fig. 7.5. In this example, node $M_{1}$ spoofs links to nodes $s, a, g$ and $j$. Node $M_{1}$ generates Hello messages and looks like the best option to be selected as an MPR by the nodes $b, d$ and $f$. After this incorrect MPR selection, node $b$ is not a selector of $h$ and vice versa. Node $d$ selects $M_{1}$ as its only MPR. The links $(b, h)$ and $(h, d)$ are not reported anymore. Thus, from the perspective of $s$, all possible paths to reach $d$ are inter-twisted. A variant of the attack can be performed by reporting a link to an inexistent node.

Invalid MPR Set. In this attack, a misbehaving node disrupts the flooding of topology control information by misbehaving during the MPR selection process. Fig. 7.3 illustrates the attack. Node $M_{1}$ is selected by $d$ as its only MPR. To perpetrate an attack, $M_{1}$ can

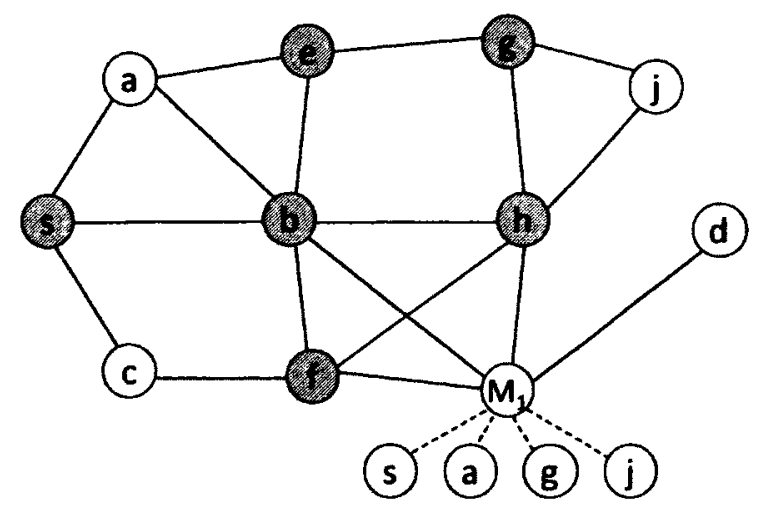

Figure 7.5: Node $s$ perspective of the network topology after a link spoofing attack. Node $M_{1}$ spoofs links to nodes $s, a, g$ and $j$. 
execute the following actions:

- It does not select an MPR set.

- It spoofs a link to an inexistent node $x$ and generates Hello messages announcing it as a one-hop neighbor and its only MPR.

- It spoofs an inexistent link to a valid node (e.g., node $a$ ) and generates Hello messages announcing that node as a one-hop neighbor and its only MPR.

- It selects node $d$ as its only MPR but do not retransmit any TC message from $d$.

Hence, TC messages from $M_{1}$ are not retransmitted by the receivers. As a consequence, node $s$ is unaware of $d$.

\section{Incorrect Relaying}

A misbehaving node can disrupt the integrity of the network by either incorrectly generating or relaying control traffic information on behalf of other nodes. Consider $M_{1}$ in Fig. 7.3 as a misbehaving node. Node $M_{1}$ is selected by $d$ as its only MPR. Then, $M_{1}$ might perform the following incorrect behaviors:

Selfish behavior. The attack is performed by a node that misbehaves and neither generates nor forwards TC messages. Fig. 7.3 illustrates an example where $M_{1}$ is an MPR but it does not relay control traffic on behalf of other nodes. As a consequence, node $d$ not receive control traffic information from other nodes. Additionally, $M_{1}$ may refuse to generate TC messages. Thus, the link between $M_{1}$ and $d$ is never reported. Notice that in an OLSR-based network, the attacker can choose not to forward any particular message, i.e., TC, MID or HNA messages.

Slanderer behavior. The list of addresses reported in each TC message can be partial (e.g., due to message size limitations). Thus, a misbehaving node can always generate TC messages without reporting all nodes in its selector table claiming that the size of the messages is not enough to include all nodes in its selector table. Therefore, when $M_{1}$ 
generates TC messages without including node $d, s$ is not be able of constructing paths to reach $d$.

Hop Limit attack. A misbehaving node can drastically modify mutable fields (e.g., TTL or Hop count values) when forwarding a TC message. If the hop limit is set equal to zero, then the scope of retransmitting the message is reduced. The attack can be performed by a misbehaving node that is either selected or not as an MPR. For instance, in Fig. 7.3, a control message is forwarded by node $b$ and received by both $e$ and $M_{1}$. Both nodes were selected by $b$ as part of its MPR set. However node $M_{1}$ forwards the message without any delay or jitter such that its retransmission arrives before that the valid message from $e$. Before forwarding, it reduces the hop limit of the message. The affected node, node $g$, will process the message and mark it as already received, ignoring future valid copies from $e$. Thus, the message with a very low hop limit will not reach the whole network.

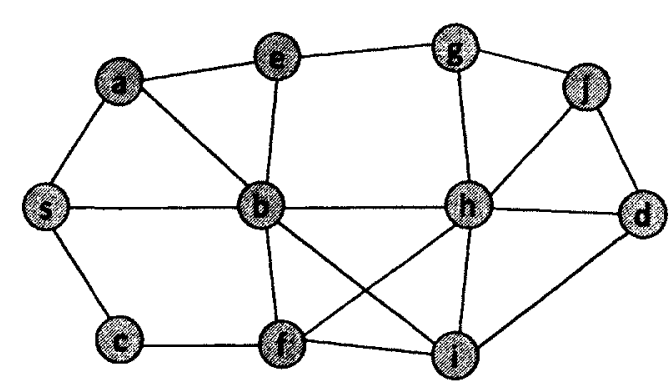

(a) $k$-CCDS and TCR parameter equal to zero.

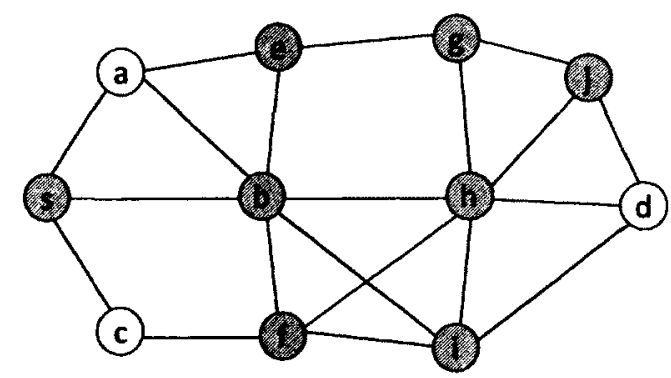

(b) $k$-RCDS and TCR parameter equal to zero.

Figure 7.6: Topology view of the network from the perspective of node $s$ and additional MPR coverage. 


\subsection{Countermeasures}

In this section, we present our countermeasures to mitigate the vulnerabilities and drawbacks of MP-OLSR, as presented in Sections 7.4.1 and 7.4.2. We present two improvements:

1. An MPR selection with additional coverage aiming to advertise more links and to increase the chances of constructing multiples node-disjoint paths.

2. A Disjoint Multipath OLSR (DM-OLSR) strategy that selects strictly node-disjoint paths. DM-OLSR privileges the MPRs with smallest selector sets to be part of the constructed routes.

Both improvements were designed to increase security in link-state multipath routing protocols for MANETs.

\subsubsection{MPR Selection with additional Coverage}

Additional coverage in the selection of the MPRs is defined in [34], as the ability of a vertex to select redundant MPRs. The selection of MPRs must be as small as possible to reduce the overhead generated by flooding the network with TC messages. In OLSR networks, the MPRs are also used as intermediate nodes to reach any destination in the system. In general, a dominating set (DS) [98] of a graph $G=(V, E)$ is a subgraph $G^{\prime}=\left(V^{\prime}, E^{\prime}\right)$, where $V^{\prime} \subset V$ and $E^{\prime} \subset E$, such that every vertex in $V-V^{\prime}$ is adjacent to some vertex in $V^{\prime}$. A connected DS (CDS) is a dominating set which also induces a connected subgraph $G^{\prime}$ of $G$. We define a MPRCDS as follows:

Definition 1 A MPRCDS is a subgraph $G^{\prime}$ of a graph $G$ such that $G^{\prime}$ is a CDS and every vertex in $G^{\prime}$ has a non empty selector set.

Nevertheless, additional coverage allows a node to advertise its presence to more nodes in the network. In this manner, extra coverage helps to maintain the integrity of the network in spite of the presence of misbehaving nodes during the execution of the protocol. 
The selection of MPRs with extra coverage is defined in the RFC3626 [34]. We named this approach a $k$-Covered-MPR selection. Fig. 7.6(a), shows an example of a network with a $k$-Covered-MPR selection. Table 7.1 shows the MPRs, their selector sets and the size of the selector sets. The MPRs form a $k$-CCDS, defined as follows:

Definition $2 A k$-CCDS is a MPRCDS of a graph $G$ such that every vertex in $G$ selects a $k$-Covered-MPR set.

However, the overhead generated by the excessive number of TC messages reduces the performance of the network. This problem is addressed by the $k$-Robust-MPR selection presented in [23]. It balances security and traffic overhead. Fig. 7.6(b) shows an example of a network with a $k$-Robust-MPR selection. The MPRs form a $k$-RCDS, defined as follows:

Definition $3 A k-R C D S$ is a MPRCDS of a graph $G$ such that every vertex in $G$ selects, when possible, a $k$-Robust-MPR set.

\subsubsection{Disjoint Multipath OLSR}

In this section, we present our Disjoint Multipath OLSR (DM-OLSR) function to construct multiple disjoint paths in an OLSR-based network. The procedure is described in function DM-OLSR. First, to increase the chances of computing multiple disjoint paths from a source node $s$ to a destination node $d$, we assume that during the topology discovery phase the nodes select their MPR set with additional coverage and set the TCR parameter to two. Then, the set of all MPRs in the network form a $k$-CCDS or a $k$-RCDS. To explain function DM-OLSR, we use the following notation:

- $d(v, w)$ : number of hops between vertex $v$ and $w$.

- $N_{1}\left(v_{q}\right)$ : one hop neighbors of $v_{q}$ i.e., $d\left(v_{q}, v_{p}\right) \leq 1$.

- $N_{\leq 2}\left(v_{q}\right)$ : nodes at distance less than or equal to two hops of $v_{q}$ i.e., $d\left(v_{q}, v_{p}\right) \leq 2$.

- $N_{2}\left(v_{q}\right)$ : two hop neighbors of $v_{q}$ i.e., $N_{\leq 2}\left(v_{q}\right) \backslash N_{1}\left(v_{q}\right)$. 
- $M\left(v_{q}\right)$ : the set $M$ is an MPR set for vertex $v_{q} \Leftrightarrow M \subseteq N_{1}\left(v_{q}\right)$ such that for every vertex $v_{q+2} \in N_{2}\left(v_{q}\right)$, $N_{1}\left(v_{q+2}\right) \cap M \neq \emptyset$.

- $S\left(v_{q}\right)$ : the set $S$ is a selector set for vertex $v_{q} \Leftrightarrow S\left(v_{q}\right) \cap N_{1}\left(v_{q}\right)$ such that for every vertex $s \in S\left(v_{q}\right), M(s) \cap v \neq \emptyset$.

- $S_{d}\left(v_{q}\right)$ : the term selectors degree of vertex $v_{q}$, refers to the cardinality of the selector set of vertex $v_{q}$.

- The path $P_{i}=\left\{v_{1}, v_{2}, \ldots v_{m}\right\}$ is invalid if there exists a pair of vertices $\left\{v_{q}, v_{q+1}\right\}$ such that $v_{q+1} \notin N_{1}\left(v_{q}\right)$ or $v_{q} \notin N_{1}\left(v_{q+1}\right)$.

- $C\left(P_{j}\right)$ : is the total cost of the path $j$. We define it as the sum of all costs $c\left(e_{q}\right)$ such that $e_{q}=\left(v_{q}, v_{q+1}\right)$ and $v_{q}, v_{q+1} \in P_{i}, m=|P|$, i.e., $C\left(P_{i}\right)=\sum_{i=1}^{m-1} c\left(e_{q}\right)$.

Function DM-OLSR receives a vertex $s$ (i.e., source node), a destination vertex $d$ (i.e., destination node), the graph $G=(V, E, c)$ and a value $t$ which is the number of disjoint paths to construct. As a result, function DM-OLSR computes a set $P$ formed by the union, if possible, of $t+1$ strictly disjoint paths from $s$ to $d$. We obtain a set $P=\left\{P_{0}, P_{1}, \ldots, P_{t}\right\}$ such as $P_{i} \cap P_{j}=\emptyset$. Therefore, we still have one more path between the source and destination even if $t$ paths fail. Function DM-OLSR works as follows:

1. First, we initialize a value $i$ equal to zero. This value $i$, represents the number of constructed paths.

2. In line 2, function AssignCost assigns to every edge $e_{q} \in E$ the cost $c\left(e_{q}\right)$ to go from vertex $v_{q}$ to $v_{q+1}$. Function AssignCost computes the cost of every edge $e_{q} \in E$. First, it assigns a cost to every vertex $v_{q} \in V$ as follows:

- The cost of vertex $s$ is equal to the number of its one-hop neighbors, see line 2 .

- If the vertex $v$ is a one-hop neighbor, then the $\operatorname{cost} c(v)$ is equal to the number of one-hop neighbors of vertex $v$. 

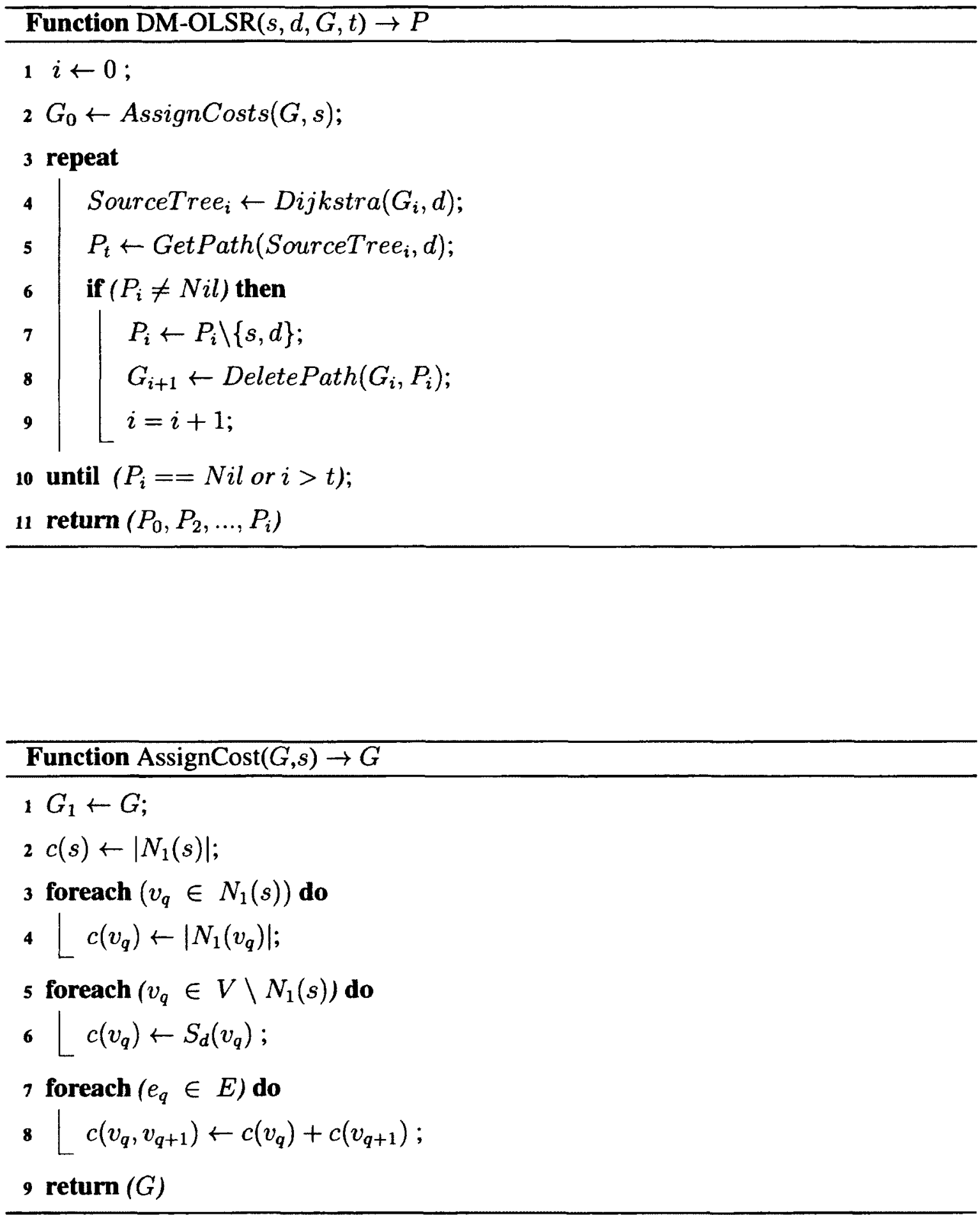
- When the vertex is not a one hop neighbor of $s$ then the cost $c(v)$ is equal to the number of elements in its selector set.

- The cost of every edge $c\left(e_{q}\right), e_{q}=\left(v_{q}, v_{q+1}\right)$, is equal to the cost of vertex $c\left(v_{q}\right)$ plus $c\left(v_{q+1}\right)$.

- Thus, function AssignCost returns a graph $G$ with costs assigned to every vertex $v_{\boldsymbol{q}} \in V$ and every edge $e_{q} \in E$.

3. In the step 4, we use Dijkstra's algorithm to obtain a path with minimum cost to reach vertex $d$.

4. If it is possible to compute a path $P_{i}$, then we delete from $G$ all the vertices included in $P_{i}$ and related edges.

5. We increase the value of $t$ and repeat the computation of paths until we obtain, if possible, $t+1$ disjoint paths.

\subsubsection{Practical Example}

As an example, in Fig. 7.5.3 the MPRs form a $k$-RCDS with TCR equal to zero. The cost of every edge is assigned by function AssignCost. The cost of vertex $s$ is equal to three. For instance, $c(s, a)$ is equal to six because node $a$ is a one-hop neighbor of node $s$ and $\left|N_{1}(a)\right|$ is equal to three. $c(e, g)$ is equal to four because $e$ and $g$ are MPRs and the size of their selector sets i.e., $S_{d}(e)$ and $S_{d}(g)$, is equal to two. $S_{d}(j)$ is equal to one and node $d$ has an empty selector set, i.e., $S_{d}(d)$ is equal to zero. Therefore, $c(j, d)$ is equal to one. If we apply function MultipathDijsktra to compute disjoint paths from vertex $s$ to reach vertex $d$ with a value of $t$ equal to two, we obtain two paths $P_{1}=\{\mathrm{s}, \mathrm{b}, \mathrm{h}, \mathrm{d}\}$ and $P_{2}=$ \{s,c,f,i,d\}. Notice that $P_{1}$ is the shortest path in term of hops, but it includes the vertices with largest selectors degree. Function MultipathDijsktra [102] does not guarantee that paths $P_{1}$ and $P_{2}$ are strictly disjoint. If we apply function DM-OLSR with $t$ equal to one, then we obtain two paths $P_{1}=\{\mathrm{s}, \mathrm{c}, \mathrm{f}, \mathrm{i}, \mathrm{d}\}$ and $P_{2}=\{\mathrm{s}, \mathrm{a}, \mathrm{e}, \mathrm{g}, \mathrm{j}, \mathrm{d}\} . P_{1}$ and $P_{2}$ are strictly disjoint paths and avoid the vertices with the largest selectors degree. Thus, the source vertex $s$ computes 1-Disjoint-Paths to reach the destination vertex $d$. 


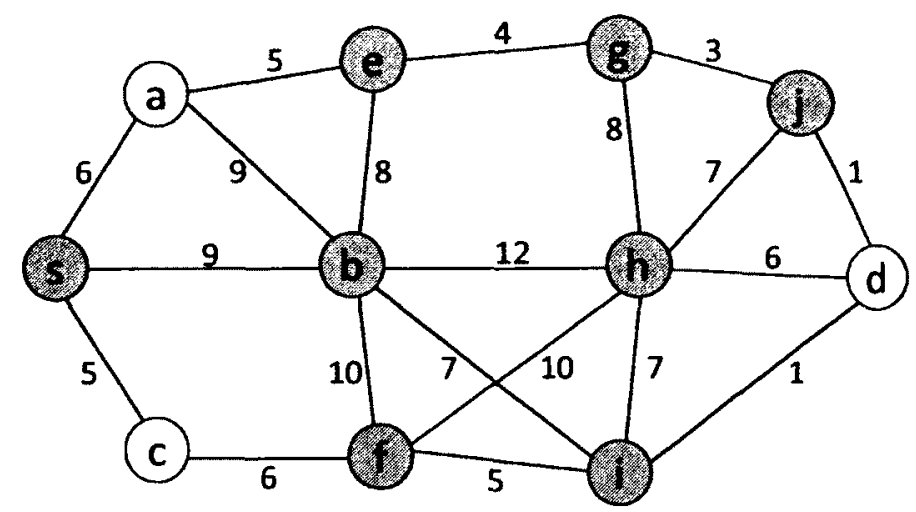

Figure 7.7: Network topology with edge cost from the perspective of $s$.

\subsubsection{Correctness of the Functions}

Theorem 3 Let $P$ be a set of $t+1$ paths obtained by node $s$ to communicate with noded by applying function DM-OLSR. Then, if $t+1>1$, then any pair of elements $P_{i}, P_{j}$ in $P$, such that $i \neq j$, are disjoint.

Proof Suppose that $P$ is a set of $t+1$ valid paths obtained by node $s$ to communicate with node $d$ by applying function DM-OLSR and there exists a pair $P_{i}, P_{j}$ in $P$ such that $i \neq j$, and $P_{i} \cap P_{j} \neq \emptyset$. Nevertheless, every time a valid path $P_{i}$ is obtained, in line 8 we eliminate from the graph $G_{i}$ all the vertices contained in $P_{i}$, except the source $s$ and the destination $d$. Hence, $G_{i}$ is equal to $G_{i-1} \backslash P_{i}-i$. Therefore, when a new path $P_{i}$ is constructed, all the elements from $P_{i-1}$ have been removed. If $i>j$, then $P_{i}$ was obtained from the graph: $G_{i}=G_{i-1} \backslash P_{1} \cup P_{2} \cup \ldots \cup P_{j} \cup \ldots \cup P_{i-1}$. If $i<j$, then $P_{j}$ is obtained from the graph: $G_{j}=G_{j-1} \backslash P_{1} \cup P_{2} \cup \ldots \cup P_{i} \cup, \ldots \cup P_{j-1}$. Therefore, $P_{i} \cap P_{j}=\emptyset$.

Theorem 4 Let $P$ be a set of $t+1$ valid paths obtained by node $s$ to communicate with node $d$ by applying function DM-OLSR and $S$ a subset of $P$ of size $t^{\prime}$, such that $t^{\prime}$ is greater than zero and less than or equal to $t$. Then, the elements in $P$ not in $S$ are still valid communication paths between $s$ and $d$.

Proof Suppose that $P$ is a set of $t+1$ valid paths obtained by node $s$ to communicate with node $d$ by applying function DM-OLSR, $S$ a subset of $P$ of size $t^{\prime}$ and $P \backslash S$ has no valid paths to reach node $d$. However, $P$ has $t+1$ valid disjoint paths. If we eliminate $t^{\prime}$ elements from $P$, then there still exists at least a valid path $P_{i}$ such that $P_{i} \cap S=\emptyset$. 
Theorem 5 Let $P$ be a set of $t+1$ valid paths obtained by node $s$ to communicate with node $d$ by applying function DM-OLSR Then, for every pair of paths $P_{i}, P_{j}$ in $P$ such that $i<j$, the total path cost of $P_{i}\left(\right.$ i.e., $\left.C\left(P_{i}\right)\right)$ assigned by function AssignCost is less than or equal to $C\left(P_{j}\right)$.

Proof Suppose there is a pair of paths $P_{i}, P_{j}$ in $\mathrm{P}$ such that $i<j$ and the total cost $C\left(P_{i}\right)$ is greater than $C\left(P_{j}\right)$. However, in function AssignCost line 4, the Dijkstra's algorithm always return the shortest path with minimum cost. Additionally, every time a new path $P_{i}$ is calculated, the nodes in the graph $G_{i}$ are eliminated by function $\operatorname{DeletePath}\left(G_{i}, P_{i}\right)$ in line 8. If a new path $P_{i+1}$ is calculated, then the total cost $C\left(P_{i+1}\right)$ must be greater than or equal to $C\left(P_{i}\right)$.

Theorem 6 Let $P$ be a set of $t+1$ valid paths obtained by node $s$ to communicate with node $d$ by applying function DM-OLSR. If there exists a valid path $P_{j}$ to reach node $d$ not in $P$, then the total cost $C\left(P_{j}\right)$ assigned by function AssignCost is higher than or equal to the total cost $C\left(P_{i}\right)$ for every path $P_{i}$ in $P$.

Proof Suppose there is a valid path $P_{j}$ not in $P$ and there exist a path $P_{i}$ in $P$ such that the total cost $C\left(P_{j}\right)$ is less than the total cost $C\left(P_{i}\right)$. According to Theorem 5 , the total path cost $C\left(P_{t+1}\right)$ in $P$ is greater than or equal to any path $P_{i}$ in $P$. Further, except for the source $s$ and the destination $d$, all the vertices in every path in $P$ have been deleted by function DeletePath. Thus, if there exist a valid path $P_{j}$ not in $P$, such that $j \geq t+1$, the $\operatorname{cost} C\left(P_{j}\right)$ assigned by function AssignCost is higher than or equal to the total cost $C\left(P_{i}\right)$ for every path $P_{i}$ in $P$.

\subsection{Simulations and Results}

In this section, we describe the experiments we conducted to measure the effectiveness of our proposed function DM-OLSR and the results we obtained. Our goal is to increase the chances of computing multiple node-disjoint routes in a OLSR-based network. For our experiments, we assume that all the nodes have the same characteristics, every node has just one interface and all the links between the nodes are bidirectional. Additionally, all the nodes have the same willingness to carry and forward traffic on behalf of other nodes, 
except for those that have been selected as misbehaving nodes. The misbehaving nodes do not collude to perform an attack.

We conducted our experiments using the NS-3 simulator [40], version 3.9. We modified the original OLSR code developed by Ros and Carneiro to implement the functions described in Sections 7.3 and 7.5.2. We tested both functions in 100 scenarios with 100 nodes each during 150 seconds. The nodes were distributed in ten clusters in an area of $1000 \mathrm{~m}$ by $1000 \mathrm{~m}$. We can consider our topology as a particular set of clusters at the same level. The nodes in each cluster follow a Zipf [39] distribution, i.e., the nodes are located in the center of each cluster with higher probability. In this topology, at least $50 \%$ of the nodes have $k$-robust MPR sets, with $k \geq 1$. The malicious nodes are selected randomly and they do not collude to perform an attack. Every node has a transmission range of 250 $\mathrm{m}$, no data traffic is generated and all the scenarios are static.

Fig. 7.8 and Fig 7.9, depict our results with $95 \%$ confidence intervals. We selected as an adversary, a malicious node with a selfish behavior (described in Section 7.4.2). In all experiments, we compared the function MultipathDijsktra proposed in MP-OLSR with TCR equal to zero or three with a MPRCDS (i.e., no additional coverage in the selection of MPR sets), function DM-OLSR with TCR equal to zero with a $k$-RCDS and $k$ equal to one (i.e., 1-Robust-MPR sets), and function DM-OLSR with TCR equal to zero with a $k$-CCDS and $k$ equal to two (i.e., 2-Covered-MPR sets). Fig 7.8(a), presents the average number of TC messages generated during the simulations and with the presence of one to five misbehaving nodes. Fig 7.8(a), shows that the number of TC messages increases when the nodes select their MPR sets with additional coverage. Our $k$-Robust-MPR selection reduces the overhead compared with the $k$-Covered-MPR selection proposed in RFC 3626 [34]. Fig 7.8(b), shows the average size of the TC message, i.e., the number of selectors included in each TC message.

In MP-OLSR, if the TCR parameter is equal to zero, then the size of each TC message is five elements in average. However, if the TCR parameter is equal to three, then 


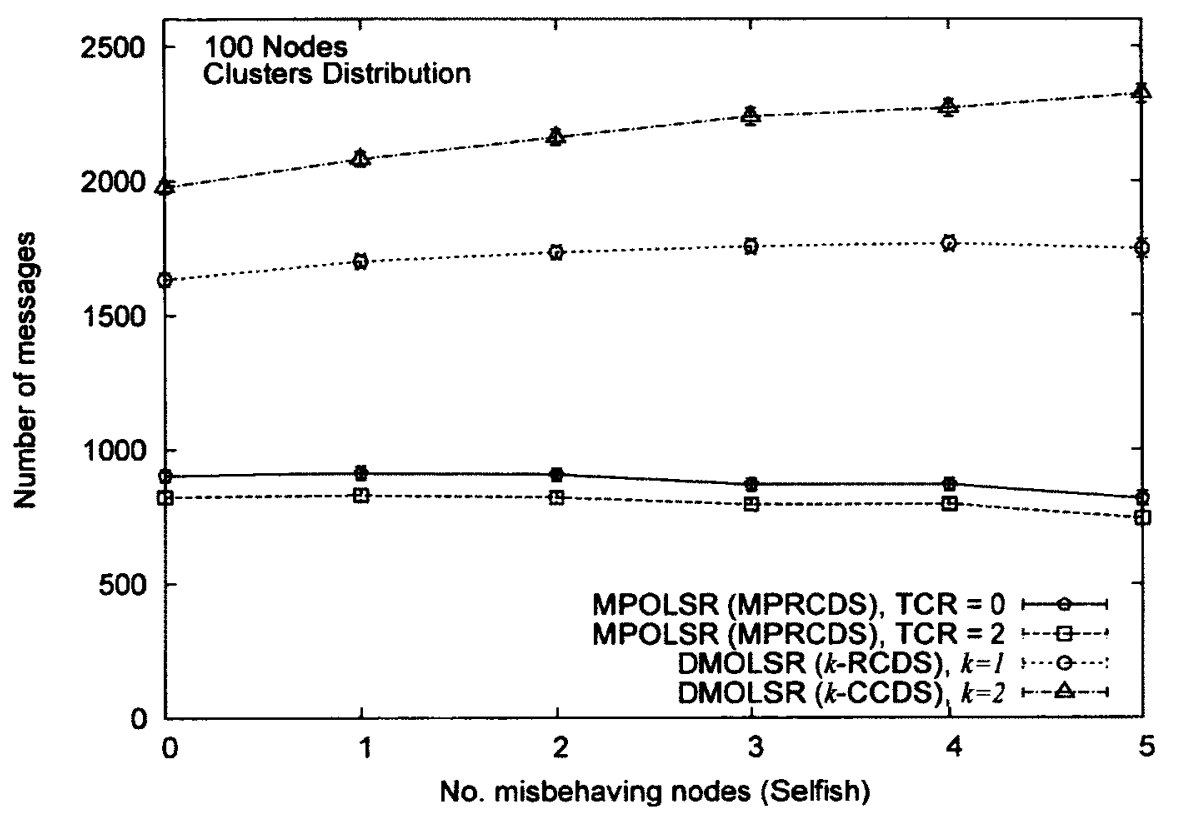

(a) Number of TC messages.

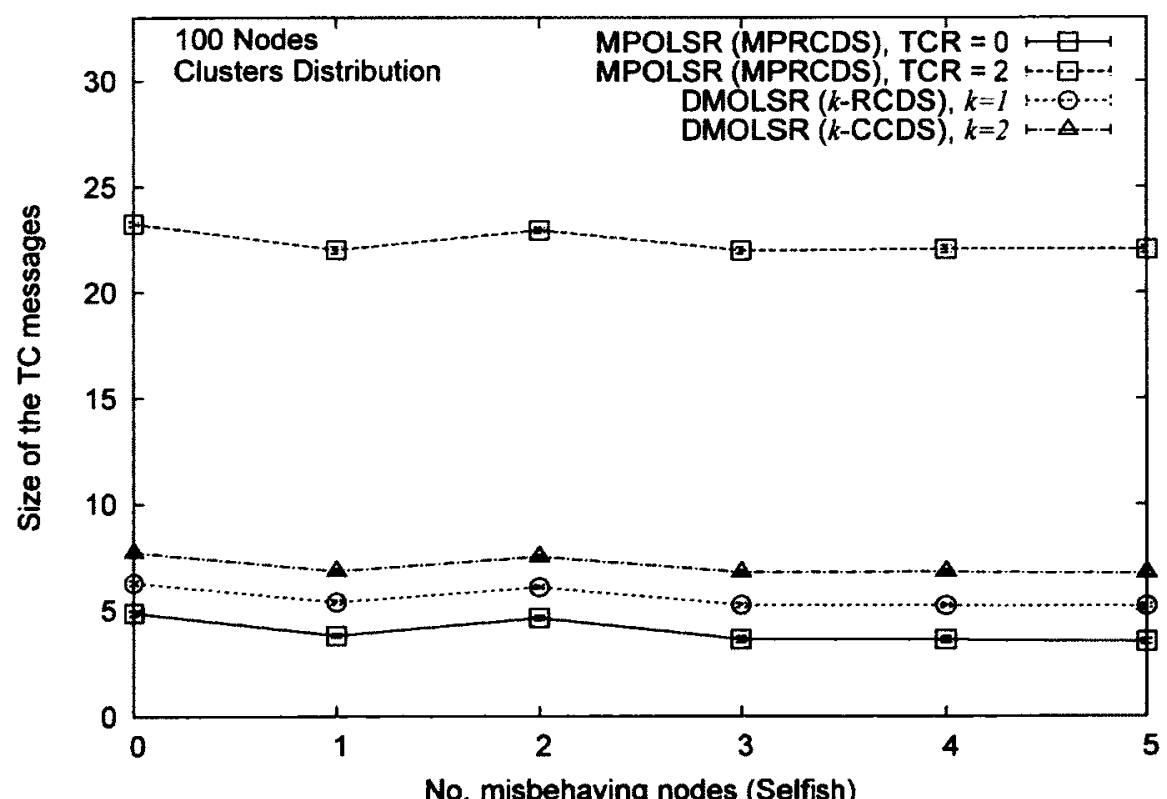

(b) Average size of the TC messages.

Figure 7.8: Simulations to compare MP-OLSR against our function DM-OLSR (95\% confidence interval). Number of TC messages and their average size. 


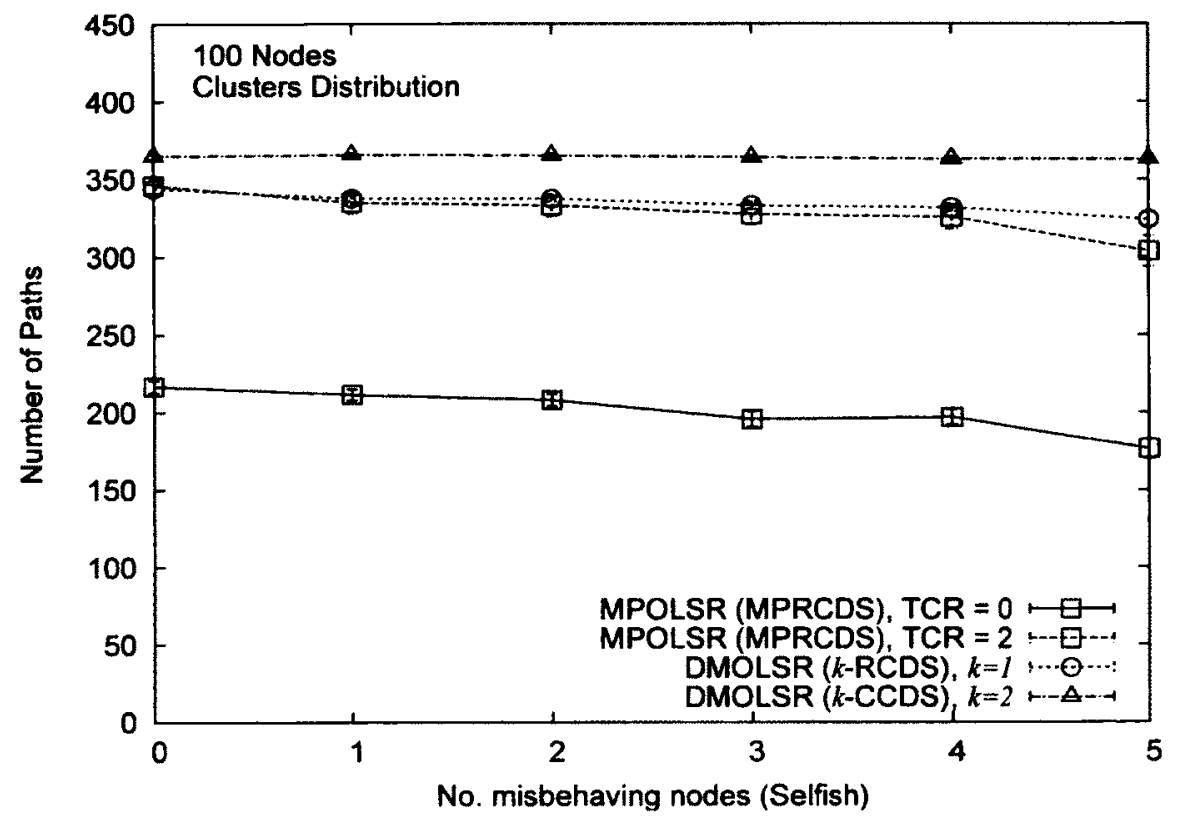

(a) Number of constructed paths.

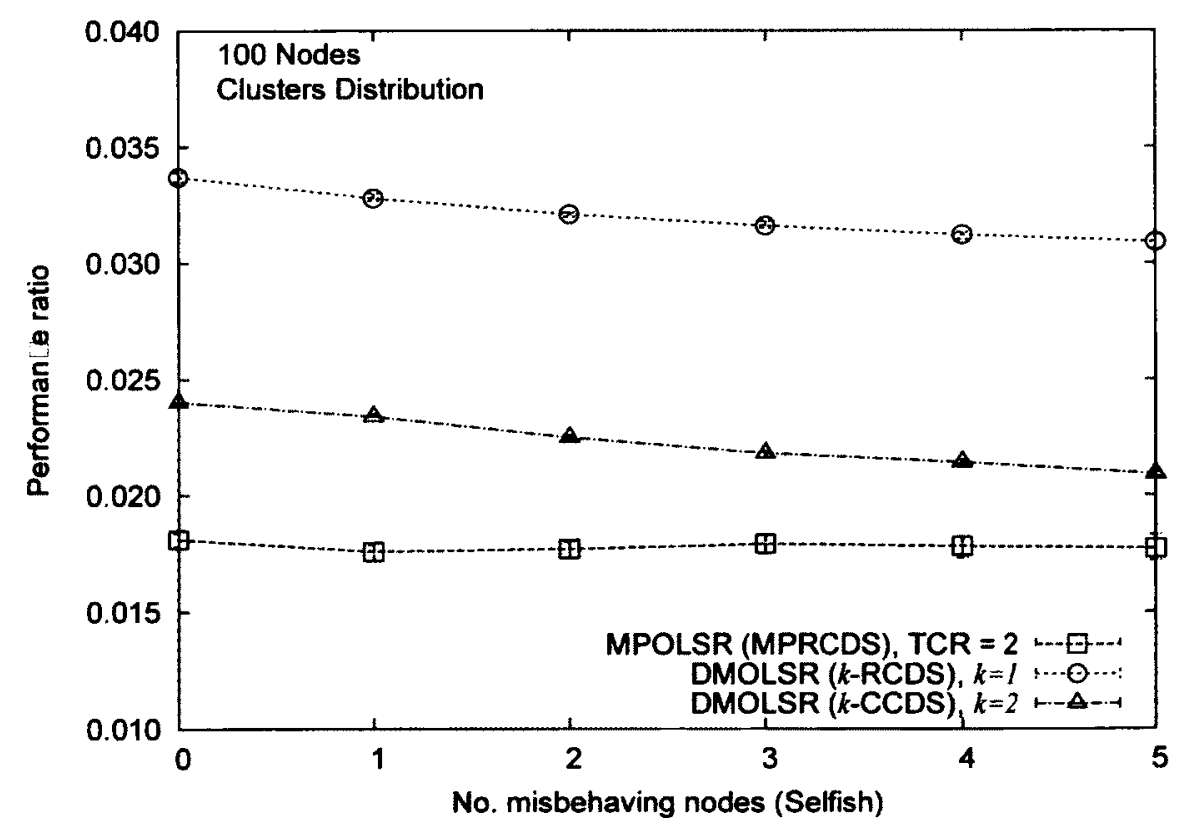

(b) Performance Ratio.

Figure 7.9: Simulations to compare MP-OLSR against our function DM-OLSR (95\% confidence interval). Number of constructed paths and performance ratio. 
the number of elements in each TC message increases to twenty two elements in average. Our DM-OLSR function with with a $k$-RCDS or a $k$-CCDS, also increases the size of the messages but in a more controlled way. Fig 7.9(a), compares the number of nodedisjoint paths constructed by all approaches. In each scenario, we counted the number of disjoint-paths that every node computes to reach every other node two or more hops away. Fig 7.9(a), shows that function DM-OLSR constructs more node-disjoint paths in a $k$-CCDS. Function MultipathDijsktra with TCR parameter equal to zero executed in a MPRCDS, is more affected by the misbehaving nodes and computes less node-disjoint paths than any other approach.

Function MultipathDijsktra with TCR parameter equal to three with no additional coverage in the selection of the MPR sets and our function DM-OLSR executed in a $k$ CCDS have similar results. However, in Fig 7.9(b), the performance ratio of function DMOLSR in a $k$-RCDS is better than any other approach. The performance ratio is equal to the number of node-disjoint paths over the amount of processed information. The processed information is equal to the number of TC messages multiply by the average size of each message. Therefore, our function MultipathDijsktra in a $k$-RCDS constructs a similar amount of node-disjoint paths but reducing the size of the messages and minimizing the overhead generated by the increased number of TC messages in the network.

\subsection{Conclusions}

We have presented the DM-OLSR (Disjoint Multipath OLSR) protocol. It provides several security improvements over other Multipath routing strategies based on OLSR. Our main goal was to achieve a flexible mechanism to compute multiple disjoint paths between any two nodes of a MANET. Furthermore, and compared to existing related work, DMOLSR improves the network topology view of the system nodes, and handles eventual flooding disruption attacks to the multipath construction mechanism. The series of simulations reported in this chapter validate our approach, and show that out solution achieves better balanced communication paths to prevents loss of messages. 


\section{Chapter 8}

\section{Conclusions and Future Work}

\subsection{Conclusions}

In this thesis, we present countermeasures to mitigate security threats in link state routing protocols based on OLSR. In OLSR, the generation and exchange of control traffic messages are important vulnerability targets. OLSR is not secure by design. Particularly, we address flooding disruption attacks in OLSR-based networks. This kind of attacks can be carried out in networks with cryptographic capabilities, e.g., a node does not select a valid MPR set or decides not to forward messages to save energy. We propose an MPR selection with additional coverage to mitigate their effect (i.e., $k$-Covered-MPR selection, Chapter 5). We compare our proposed mechanism against the heuristic presented in the RFC3626 [34] (i.e., $k$-Robust-MPR selection, Chapter 5) to compute MPR sets with additional coverage. Our results show that our $k$-Robust-MPR selection reduces the overhead created by additional control information flooded in the network by the $k$ Covered-MPR selection and can help to mitigate the effect of flooding disruption attacks in OLSR. Additionally, we review security issues in hierarchical and multipath OLSRbased routing protocols. Besides OLSR, we review security risks in the Hierarchical OLSR (HOLSR) [96] protocol proposed by Villasenor et al. and the Multipath OLSR (MP-OLSR) [103, 104, 105, 102] protocol, proposed by Yi et al. We address the effect of flooding disruption attacks in these OLSR-based routing protocols. Additional coverage in the MPR computation may be implemented in a cryptographic capable network to enforce security. The MPR computation with or without additional coverage is local and no additional change in the control traffic messages is necessary. Therefore, our proposed countermeasures can be implemented in other proactive link-state routing protocols based on the MRPs as a flooding mechanism. 
Mobility affects the accuracy of the routing tables, i.e., nodes need to receive new control messages to update their routing tables. This process is also affected by the presence of misbehaving nodes. In this work, we tested our solutions only in static scenarios to identify how the presence of misbehaving nodes affect the construction of routing tables. As part of future work, mobility may be added as a new component to evaluate the effectiveness of our solutions. Additional coverage in the selection of MPRs, may help to reduce the time to detect changes in the topology due to mobility. This thesis is based on the work presented in $[23,24,25]$.

\subsubsection{Summary of Contributions and Results}

Our contributions and results can be summarized as follows:

- In Chapter 3, we show vulnerabilities in OLSR. We present a taxonomy of flooding disruption attacks. The taxonomy presented in Section 3.2, divides the flooding disruption attacks in two categories: Incorrect MPR Selection and Incorrect Relaying.

- In Chapter 5, we analyzed the effect of control traffic attacks in OLSR and the selection of MPR sets with additional coverage to mitigate their effect. The MPR selection with additional coverage is presented in RFC 3626 [34], we name it $k$ covered-MPR selection. However, additional coverage reduces the performance of the network due to additional control traffic information (i.e., TC messages). We propose a $k$-robust-MPR selection. In a $k$-robust-MPR selection a node computes, when possible, $k+1$ disjoint MPR sets to guarantee that even if $k$ of the selected MPR sets become invalid, the remaining set is still a valid MPR set. Our proposed MPR selection offers equivalent protection against control traffic attacks but reducing the overhead generated by additional control traffic information. We compare the $k$-covered-MPR and $k$-robust-MPR selection in the presence of misbehaving nodes. We measured the number of nodes with complete routing tables after the execution of the OLSR protocol. Our experiments show that our $k$-robust-MPR selection reduces the amount of traffic generated by the $k$-covered-MPR selection, and offers equivalent protection against control traffic attacks. Additionally, our $k$ robust-MPR selection, increases the performance ratio of the number of nodes with 
complete routing tables over the number of topology control messages.

- In Chapter 6, we present security threats in HOLSR. HOLSR uses TC messages for intra-cluster communications and implements Hierarchical TC (HTC) messages for inter-cluster communications. HTC messages are flooded exclusively by the MPRs. Therefore, the inter-cluster communication is also affected by flooding disruption attacks. In [24], we proposed to mitigate the effect of the attacks against HTC messages by selecting MPR sets with additional coverage (i.e., $k$-Robust-MPR and $k$-Covered-MPR selections). The countermeasures that we propose, can be implemented in other hierarchical approaches based on OLSR, e.g., Cluster OLSR(COLSR) [88] proposed by Ros et al., the Multi-level OLSR routing using the HNA extension (MORHE) proposed in [97] by Voorhaen et al. or the Tree Clustering $[17,16]$ mechanism proposed by Baccelli.

- In Section 6.6.2, we present algorithm HCCD based on hash chains to enforce the cluster formation phase in HOLSR. In HOLSR, Cluster ID Announcement (CID) messages are implemented to organize the network in clusters. A misbehaving node may maliciously alter mutable fields (e.g., hop count) in CID messages to unbalance the distribution of nodes in clusters. HCCD allows a node to detect and discard invalid CID messages. Our algorithm can be implemented in other hierarchical approaches that use messages with mutable fields to organize the network in clusters. A variation of the CID attack is presented in Section 6.6.3. $\mathrm{HCCD}_{2}$ is a variation of the HCCD algorithm to handle a misbehaving node with more than one interface that tunnels valid CID messages between cluster at different levels, i.e., a wormhole attack.

- In Chapter 7, we analyze vulnerabilities in multipath OLSR-based networks. MPOLSR is based on the MPR flooding mechanism to distribute control traffic information in the network. However, MPRs report partial link state information. Therefore, MP-OLSR nodes only acquire a partial view of the network. We present function DM-OLSR (Disjoint Multipath OLSR) to compute multiple node disjoint paths between any two nodes of a MANET. Furthermore, we compare with function MultipathDijsktra proposed in MP-OLSR. Our function DM-OLSR, uses additional 
coverage to improve the network topology view of the network and mitigate the effect of flooding disruption attacks during the topology discovery and the route computation phases. We show that our solution function DM-OLSR increases the chances of constructing multiple node disjoint paths when additional coverage in the selection of MPR is used. In MP-OLSR redundant information in the TC messages is proposed to increase the chances of discovering paths. Our results show that redundant information mitigates the effect to flooding disruption attacks but the size of each TC message increase considerably. Our function DM-OLSR computes disjoint paths with a better performance ratio than the function MultipathDijsktra proposed in MP-OLSR.

\subsection{Future Work}

In this section, we present open problems for further research based on the results presented in Chapters 5, 6 and 7.

\subsubsection{Phantom node attack in OLSR}

The phantom node attack is presented in Chapter 3 as an special case of the node spoofing attack. Nevertheless, we study this attack in more detail because it can be launched to affect the $k$-robust-MPR selection proposed in Chapter 5. Thus, if an attacker reports non-existent one-hop neighbors it prevents other valid one-hop neighbors to select disjoint MPR sets. The $k$-covered-MPR approach proposed in the RFC3626 [34] for an MPR selection with additional coverage is more resilient to this attack, however the performance of the network is reduced due to the increased amount of topology control messages. We aim to present a variation of our disjoint MPR selection to mitigate the effect of Phantom node attacks and to reduce the overhead generated by the selection of MPR sets with additional coverage.

\subsubsection{Locally proactive OLSR-based networks}

In an OLSR-based network, every node computes its MPR set based on the same algorithm. We propose that security can be enhanced if nodes calculate their MPR set using 
local information. For instance in HOLSR, nodes in higher levels and with superior capabilities might increase the level of security. In addition, an MPR might consider to retransmit control traffic messages following a non source dependant mechanism, i.e., the MPRs retransmit TC messages even if the sender is not included in its selector table. This decision might be made based on other premises, e.g., in a hierarchical approach, the MPRs retransmit all TC messages from a cluster head one-hop away. The algorithm and the parameters to decide which MPR selection or forwarding mechanism are the most appropriate are both open problems.

\subsubsection{MPR Selection with Additional Coverage in Dynamic Networks}

In our work, we study the selection of MPRs with additional coverage in static ad hoc networks. As future work, mobility should be considered to measure how the construction of routing tables is affected by a malicious node that perpetrates a flooding disruption attack in dynamic ad hoc network.

\subsubsection{Colluding Flooding Disruption Attacks in OLSR}

At this point, we have considered non-colluding attacks. As further research we will analyze attacks when two attackers collude to perform flooding disruption attacks in OLSR. Therefore, countermeasures against more sophisticated attacks when nodes collude to perform a flooding disruption attack are part of our future work.

\subsubsection{Handling other cluster formation attacks in HOLSR}

In Chapter 6 we present security threats during the cluster formation phase. We present algorithms $\mathrm{HCCD}$ and $\mathrm{HCCD}_{2}$ to detect and discard incorrect $\mathrm{CID}$ messages. However, adversaries with more capabilities will require more complex solutions. Then, as future research we propose to consider the following attacks:

- An adversary might replay an old valid message. As a consequence the receiver nodes can join an inexistent cluster head.

- An adversary with the capability of impersonating a valid cluster head might generate valid CID messages. 
- The adversary can also retransmit a valid CID message without modifying the hop count value.

- Colluding nodes may perpetrate a wormhole attack. Two nodes tunnel valid CID messages between two different clusters at the same level.

Therefore, the solutions should consider adversaries with more capabilities to perpetrate variations of the CID attack. These solutions may be based on hash chains but complemented with other cryptographic mechanisms such as: certificates, signatures, time stamps, etc. The design and implementation of an algorithm to prevent this kind of attacks is part of future work. 


\section{Acronyms}

AODV Ad hoc On-demand Distance Vector.

C-OLSR Cluster OLSR.

CDS Connected Dominating Set.

CID Cluster ID Announcement.

DM-OLSR Disjoint Multipath OLSR.

DSDV Destination-Sequenced Distance Vector.

DSR Dynamic Source Routing.

FSR Fish Eye Routing.

LMR Light-Weight Mobile Routing.

LP-HOLSR Locally Proactive HOLSR.

MORHE Multi-level OLSR Routing using the HNA Extension.

MP-OLSR Multipath OLSR.

OLSR Optimized Link State Routing.

TBRPF Topology dissemination Based on Reverse-Path Forwarding.

TCR TC redundancy.

TORA Temporally Ordered Routing Algorithm.

WRP Wireless Routing Protocol.

ZHLS Zone-based Hierarchical Link State.

ZRP Zone Routing Protocol. 


\section{Bibliography}

[1] Hipercom project, Object-Oriented OLSR (OOLSR), Project web page available in http://hipercom.inria.fr/oolsr/. INRIA, France, August 2004.

[2] Hipercom project, Simple Multicast OLSR (SMOLSR), Project web page available in http://hipercom.inria.fr/SMOLSR-MOLSR/. INRIA, France, November 2004.

[3] No Overhead Autoconfiguration OLSR (NOA-OLSR), Project web page available in http://www2.net.ie.niigata-u.ac.jp/olsr-e.php. Niigata University, Niigata, Japan, August 2004.

[4] The NRL OLSR Routing Protocol Implementation, Project web page available in http://cs.itd.nrl.navy.mil/work/olsr/index.php. The Networks and Communication Systems Branch of the Information Technology Division (ITD) at the U.S. Naval Research Laboratory, USA, August 2004.

[5] OLSRd, OLSR daemon, UniK, Project web page available in http://www.olsr.org/. University of Oslo, Oslo, Norway, March 2011.

[6] R. Abdellaoui and J.-M. Robert. SU-OLSR: A new solution to thwart attacks against the OLSR protocol. In 4th Conference on Security in Network Architectures and Information Systems (SAR-SSI), Luchon, France, June, 22 - 262009.

[7] M. Abolhasan, T. Wysocki, and E. Dutkiewicz. A review of routing protocols for mobile ad hoc networks. Ad Hoc Networks, 2(1):1-22, January 2004.

[8] C. Adjih, E. Baccelli, T.H. Clausen, P. Jacquet, and G. Rodolakis. Fish eye OLSR scaling properties. Journal of Communications and Networks, 6(4):352-361, December 2004.

[9] C. Adjih, T. Clausen, A. Laouiti, P. Muhlethaler, and D. Raffo. Securing the OLSR routing protocol with or without compromised nodes in the network. Technical Report, INRIA RR-5494, HIPERCOM project, INRIA Rocquencourt, France, February 2005 .

[10] C. Adjih and L. Viennot. Computing connected dominated sets with multipoint relays. Ad Hoc and Wireless Sensors Networks, 1:27 - 39, March 2005.

[11] A. Adnane, C. Bidan, and R.T. de Sousa Jr. Validation of the OLSR routing table based on trust reasoning. In International Workshop on Trust in Mobile Environments, pages 1-12, 2008. 
[12] A. Adnane, R.T. de Sousa Jr., C. Bidan, and L. Mé. Autonomic trust reasoning enables misbehavior detection in OLSR. In ACM Symposium on Applied computing (SAC), pages 2006-2013, New York, USA, 2008.

[13] D.-P. Agrawal and Q.-A. Zeng. Introduction to Wireless and Moile Systems. Thomson, 2ed edition, 2006.

[14] H. Aiache, F. Haettel, L. Lebrun, and C. Tavernier. Improving security and performance of an ad hoc network through a multipath routing strategy. Journal of Computer Virology, 4:267-278, 2008.

[15] P. Arce, J.C. Guerri, A. Pajares, and O. Lázaro. Performance evaluation of video streaming over ad hoc networks using flat and hierarchical routing protocols. Mobile Networks and Applications, 13(3-4):324-336, 2008.

[16] E. Baccelli. OLSR scaling with hierarchical routing and dynamic tree clustering. In IASTED International Conference on Networks and Communication Systems (NCS), 2006.

[17] E. Baccelli. OLSR trees: A simple clustering mechanism for OLSR. Challenges in Ad Hoc Networking, IFIP International Federation for Information Processing, 197:265-274, 2006.

[18] H. Badis and K. Al Agha. QOLSR multi-path routing for mobile ad hoc networks based on multiple metrics: bandwidth and delay. In Vehicular Technology Conference, IEEE, volume 4, pages 2181 - 2184, May 2004.

[19] M. Barbeau and E. Kranakis. Principles of ad hoc networking. John Wiley and Sons, Ltd , ISBN: 9780470032909, 2007.

[20] K.A. Bradley, S. Cheung, N. Puketza, B. Mukherjee, and R.A. Olsson. Detecting disruptive routers: a distributed network monitoring approach. IEEE Network, 12(5):50-60, September 1998.

[21] S. Buchegger and J.-Y. Le Boudec. Performance analysis of the confidant protocol. In 3rd ACM international symposium on mobile ad hoc networking and computing (MobiHoc), pages 226-236, New York, NY, USA, 2002.

[22] P. Carmi, M.J. Katz, and N. Lev-Tov. Covering points by unit disks of fixed location. In 18th International Symposium on Algorithms and Computation (ISAAC), volume 4835, pages 644-655, Sendai, Japan, December 17 - 192007.

[23] G. Cervera, M. Barbeau, J. Garcia-Alfaro, and E. Kranakis. Mitigation of topology control attacks in OLSR networks. In 5th International Conference on Risks and Security of Internet and Systems (CRISIS), Jean-Marc Robert, editor, pages 81-88, Montreal, Canada, October 10 - 13, 2010. 
[24] G. Cervera, M. Barbeau, J. Garcia-Alfaro, and E. Kranakis. Mitigation of flooding disruption attacks in HOLSR networks. In 9th Annual Conference on Communication Networks and Services Research Conference (CNSR), Ottawa, ON, Canada, May 2 - 52011.

[25] G. Cervera, M. Barbeau, J. Garcia-Alfaro, and E. Kranakis. Preventing the Cluster Formation Attack Against the Hierarchical OLSR Protocol: Invited Talk. In 4th Canada-France MITACS Workshop on Foundations \& Practice of Security (FPS), Paris, France, May 12 - 132011.

[26] T. Clausen and C. Dearlove. RFC5497: Representing Multi-Value Time in Mobile Ad Hoc Networks (MANETs), http://www.ietf.org/rfc/rfc5497.txt, March 2009.

[27] T. Clausen, C. Dearlove, and B. Adamson. RFC5148: Jitter Considerations in Mobile Ad Hoc Networks (MANETs), http://www.ietf.org/rfc/rfc5148.txt, February 2008.

[28] T. Clausen, C. Dearlove, and J. Dean. I-D: MANET Neighborhood Discovery Protocol (NHDP), work in progress, March 2008.

[29] T. Clausen, C. Dearlove, J. Dean, and C. Adjih. RFC5444: Generalized mobile ad hoc network (manet) packet/message format, http://www.ietf.org/rfc/rfc5444.txt, February 2009.

[30] T. Clausen, C. Dearlove, and P. Jacquet. Optimized link state routing protocol version 2(OLSRv2), RFC3666, Work in progress. Project Hipercom, INRIA, France, Internet Draft, http://bgp.potaroo.net/ietf/all-ids/draft-ietf-manet-olsrv2-11.txt, October 2010 .

[31] T. Clausen and U. Herberg. Security Issues in the Optimized Link State Routing Protocol version 2 (OLSRv2). Research Report RR-7218, INRIA, France, March 2010.

[32] T. Clausen and U. Herberg. Vulnerability analysis of the optimized link state routing protocol version 2 (OLSRv2). In Wireless Communications, Networking and Information Security (WCNIS), , pages 628 - 633, June 2010.

[33] T. Clausen, U. Herberg, and J. Milan. Digital signatures for admittance control in the optimized link state routing protocol version 2. Research Report RR-7216, INRIA, France, February 2010.

[34] T. Clausen and P. Jacquet. Optimized link state routing protocol (OLSR), RFC3626. IETF Internet Draft, http://www.ietf.org/rfc/rfc3626.txt, October 2003.

[35] M.S. Corson and A. Ephremides. A distributed routing algorithm for mobile wireless networks. In ACM/Baltzer Wireless Networks, vol. 1 (1) 61 - 81, 1995. 
[36] Communications Research Centre Canada (CRC). CRC OLSR. Project Web Page available in http://www.crc.gc.ca/en/html/manetsensor/home/software/software, March, 2011.

[37] G. Călinescu, I. Măndoiu, P.-J. Wan, and A. Zelikovsky. Selecting forwarding neighbors in wireless ad hoc networks. In 5th International Workshop on Discrete Algorithms and Methods for Mobile Computing and Communications (DIALM), pages $34-43$, New York, USA, 2001.

[38] F. Cuppens, N. Cuppens-Boulahia, T. Ramard, and J. Thomas. Misbehaviors detection to ensure availability in OLSR. In Mobile Sensor Networks (MSN), volume 4864 of Lecture Notes in Computer Science, pages 799-813. Springer, 2007.

[39] L. Devroye. Non-uniform random variate generation. Springer-Verlag, New York, 1986.

[40] T. Henderson et. al. The NS-3 network simulator. Software package retrieved from http://www.nsnam.org/, 2011.

[41] A. Fourati and K. Agha. A shared secret-based algorithm for securing the OLSR routing protocol. Telecommunication Systems, 31:213-226, 2006.

[42] Zygmunt J. Haas and Marc R. Pearlman. ZRP: a hybrid framework for routing in ad hoc networks. In Ad hoc networking, pages 221-253, Addison-Wesley Longman Publishing Co., Inc. Boston, MA, USA, 2001.

[43] A. Hajami, K. Oudidi, and M. Elkoutbi. An enhanced algorithm for MANET clustering based on multi hops and network density. In 10th Annual International Conference on New Technologies of Distributed Systems (NOTERE),, pages 181-188, 2010.

[44] F. Hong, L. Hong, and C. Fu. Secure olsr. International Conference on Advanced Information Networking and Applications, 1:713-718, 2005.

[45] Y.-C. Hu, D. B. Johnson, and A. Perrig. Sead: secure efficient distance vector routing for mobile wireless ad hoc networks. Ad Hoc Networks, 1(1):175 - 192, 2003.

[46] J. R. Hughes, T. Aura, and M. Bishop. Using conservation of flow as a security mechanism in network protocols. In IEEE Symposium on Security and Privacy, pages 123-141, 2000.

[47] P. Jacquet, P. Muhlethaler, T. Clausen, A. Laouiti, A. Qayyum, and L. Viennot. Optimized link state routing protocol for ad hoc networks. In IEEE International Multi Topic Conference (IEEE), Technology for the 21st Century, pages 62-68, Pakistan, December 2001. 
[48] L. Ji and M. S. Corson. A lightweight adaptive multicast algorithm. In Proceedings of the IEEE Global Telecommunications Conference (GLOBECOM), volume 2, pages 1036-1042, Sydney, Australia, 1998.

[49] M. Joa-Ng and I.-T. Lu. A peer-to-peer zone-based two-level link state routing for mobile ad hoc networks. IEEE Journal on Selected Areas in Communications, 17(8):1415-1425, 1999.

[50] D. B. Johnson and D. A. Maltz. Dynamic source routing in ad hoc wireless networks. In Tomasz Imielinski and Henry F. Korth, editors, Mobile Computing of The Kluwer International Series in Engineering and Computer Science, volume 353, pages 153-181. Springer US, 1996.

[51] J.F.Ch. Joseph, A. Das, and B-S. Lee. Security threats in ad hoc routing protocols. In Guide to Wireless Ad Hoc Networks, Computer Communications and Networks, pages 1-18. Springer London, 2009.

[52] B. Kannhavong, H. Nakayama, N. Kato, A. Jamalipour, and Y. Nemoto. A study of a routing attack in OLSR-based mobile ad hoc networks. International Journal of Communication Systems, 20(11):1245-1261, 2007.

[53] B. Kannhavong, H. Nakayama, Y. Nemoto, N. Kato, and A. Jamalipour. A survey of routing attacks in mobile ad hoc networks. IEEE Wireless Communications, 14(5):85-91, 2007.

[54] A.R. Khakpour, M. Laurent-Maknavicius, and H. Chaouchi. WATCHMAN: An Overlay Distributed AAA Architecture for Mobile Ad hoc Networks. In Third International Conference on Availability, Reliability and Security (ARES), pages 144 -152 , March 2008.

[55] M. Kun, Y. Jingdong, and R. Zhi. The research and simulation of multipath-OLSR for mobile ad hoc network. In IEEE International Symposium on Communications and Information Technology (ISCIT), pages 540 - 543, October 2005.

[56] A. Kush and C. Hwang. Proposed protocol for hash-secured routing in ad hoc networks. Masaum Journal of Computing (MJC), ISSN 2076-0833, 1:221-226, 2009.

[57] L. Lamport. Password authentication with insecure communication. Communications of the ACM, 24:770-772, November 1981.

[58] D. Lang. On the evaluation and classification of routing protocols for mobile ad hoc networks. PhD thesis, Technical University Munich (TUM), Munich, Germany, 2006.

[59] A. Laouiti, P. Jacquet, P. Minet, L. Viennot, T. Clausen, and C. Adjih. Multicast Optimized Link State Routing. Research Report RR-4721, INRIA, France, 2003. 
[60] J. Liu, X. Hong, J. Kong, Q. Zheng, N. Hu, and P. Bradford. A hierarchical anonymous routing scheme for mobile ad-hoc networks. In Military Communications Conference (MILCOM), 2006.

[61] E. Gregori M. Conti and G. Maselli. Improving the performability of data transfer in mobile ad hoc networks. In 2th Annual IEEE Communications Society Conference on Sensor and Ad Hoc Communications and Networks (IEEE SECON), pages 153 - 163, September 2005.

[62] J. Macker, I. Downard, J. Dean, and B. Adamson. Evaluation of distributed cover set algorithms in mobile ad hoc network for simplified multicast forwarding. ACM SIGMOBILE Mobile Computing and Communications Review, 11(3):1-11, 2007.

[63] J.P. Macker, J. Dean, and W. Chao. Simplified multicast forwarding in mobile ad hoc networks. In IEEE Military Communications Conference (MILCOM), volume 2, pages 744 - 750, October 2004.

[64] J. D. Marshall. An analysis of the secure routing protocol for mobile ad hoc network route discovery: using intuitive reasoning and formal verification. Technical report, Florida State University, USA, 2003.

[65] S. Marti, T. J. Giuli, K. Lai, and M. Baker. Mitigating routing misbehavior in mobile ad hoc networks. In 6th annual international conference on Mobile computing and networking (MobiCom), pages 255-265, New York, NY, USA, 2000.

[66] S. McCanne, S. Floyd, K. Fall, K. Varadhan, et al. The network simulator: NS-2. Software package retrieved from http://www.isi.edu/nsnam/ns/, 1997.

[67] Z. Min and Z. Jiliu. Cooperative black hole attack prevention for mobile ad hoc networks. In International Symposium on Information Engineering and Electronic Commerce (IEEC), pages 26-30, May 2009.

[68] P. Minet and A. Laouiti. Multicasting in mobile ad hoc networks. In Guide to Wireless Ad Hoc Networks, Computer Communications and Networks, pages 1-24. Springer London, 2009.

[69] J. Moy. Open Shortest Path First (OSPF) version 2, RFC2328, IETF Internet Draft, http://www.ietf.org/rfc/rfc2328.txt, April 1998.

[70] S. Mueller, R. P. Tsang, and D. Ghosal. Multipath routing in mobile ad hoc networks: issues and challenges. In Performance Tools and Applications to Networked Systems, volume 2965 of LNCS, pages 209-234. Springer-Verlag, 2004.

[71] S. Murthy and J.J. Garcia-Luna-Aceves. A routing protocol for packet radio networks. In Proceedings of the 1st annual international conference on Mobile computing and networking (MobiCom), pages 86-95, New York, NY, USA, 1995. 
[72] A. M. Naimi, C. Adjih, P. Minet, and G. Rodolakis. Simulation-based comparison of three wireless multicast routing protocols: MOST, MOLSR and SMOLR. In AdHoc, Mobile and Wireless Networks, volume 6288 of Lecture Notes in Computer Science, pages 1-14, 2010.

[73] S. Narayanappa and P. Vojtechovsky. An improved approximation factor for the unit disk covering problem. In Canadian Conference on Computational Geometry (CCCG), pages 15-18. Queen's University, Kingston, Ontario., Aug 14-16, 2006.

[74] D. Nguyen and P. Minet. Scalability of the OLSR Protocol with the Fish Eye Extension. In 6th International Conference on Networking (ICN), page 88, Washington, DC, USA, April 2007. IEEE Computer Society.

[75] R. Ogier, F. Templin, and M. Lewis. Topology Dissemination Based on ReversePath Forwarding (TBRPF), RFC3684. Network Working Group, February 2004.

[76] D. Palma and M. Curado. Inside-Out OLSR Scalability Analysis. In 8th International Conference on Ad-Hoc, Mobile and Wireless Networks (ADHOC-NOW), pages 354-359, Heidelberg, Germany, 2009.

[77] V. D. Park and M. S. Corson. A highly adaptive distributed routing algorithm for mobile wireless networks. In IEEE Annual Joint Conference of the Computer and Communications Societies, page 1405, Los Alamitos, CA, USA, 1997.

[78] G. Pei, M. Gerla, and T.-W. Chen. Fish eye routing: A routing scheme for ad hoc wireless networks. IEEE International Conference on Communications, 1:70 - 74, 2000. Institute of Electrical Engineers INC. (IEEE), USA , ISSN 0536-1486.

[79] C. Perkins, E. Belding-Royer, and S. Das. Ad hoc on-demand distance vector (AODV) routing. RFC3561, RFC Editor, United States, 2003.

[80] Ch. E. Perkins and P. Bhagwat. Highly dynamic Destination-Sequenced DistanceVector routing (DSDV) for mobile computers. In Conference on Communications Architectures, Protocols and Aplications (SIGCOMM), pages 234-244, New York, NY, USA, 1994.

[81] P. P. Pham. Multi-path routing with load balance and cross-layer design in MANETs. PhD thesis, University of South Australia, Australia, August 2004.

[82] P.P. Pham and S. Perreau. Performance analysis of reactive shortest path and multipath routing mechanism with load balance. In 22th Annual Joint Conference of the IEEE Computer and Communications (INFOCOM). IEEE Societies, volume 1, pages $251-259$, March 2003.

[83] D. Raffo. Security schemes for the OLSR protocol for ad hoc networks. $\mathrm{PhD}$ thesis, L'Université Paris 6 - Pierre et Marie Curie, INRIA Roquencourt, France, September 2005 . 
[84] D. Raffo, C. Adjih, T. Clausen, and P. Muhlethaler. Securing OLSR using node locations. In Proceedings of 2005 European Wireless (EW), pages 437-443, Nicosia, Cyprus, April 10-13 2005.

[85] S. Rana and A. Kapil. Defending against node misbehavior to discover secure route in olsr. In Information Processing and Management, volume 70 of Communications in Computer and Information Science, V. V. Das and R. Vijayakumar and N.C. Debnath and J. Stephen and N. Meghanathan and S. Sankaranarayanan and P.M. Thankachan and F.L. Gaol and N. Thankachan, editors, pages 430-436. Springer Berlin Heidelberg, 2010.

[86] G. Rodolakis, A. Naimi, and A. Laouiti. Multicast overlay spanning tree protocol for ad hoc networks. In Wired/Wireless Internet Communications, volume 4517 of Lecture Notes in Computer Science, F. Boavida and E. Monteiro and S. Mascolo and Y. Koucheryavy, editors, pages 290-301. Springer Berlin Heidelberg, 2007.

[87] F.J. Ros. UM-OLSR, an implementation of the OLSR (Optimized Link State Routing) protocol for the NS-2 network simulator. Department of Information and Communications Engineering, University of Murcia, Spain, Software package retrieved from http://masimum. inf. um.es/um-olsr/html, August 2010.

[88] F.J. Ros and P.M. Ruiz. Cluster-based OLSR extensions to reduce control overhead in mobile ad hoc networks. In International Conference on Wireless Communications and Mobile Computing, pages 202-207, 2007.

[89] K. Shaukat and V. R. Syrotiuk. Locally proactive routing protocols. In Ioanis Nikolaidis and Kui Wu, editors, Ad-Hoc, Mobile and Wireless Networks, volume 6288 of Lecture Notes in Computer Science, pages 67-80. Springer, 2010.

[90] A. Srinivas and E. Modiano. Minimum energy disjoint path routing in wireless adhoc networks. In 9th Annual International Conference on Mobile Computing and Networking (MobiCom), pages 122-133, New York, NY, USA, 2003. ACM.

[91] W. Stallings. Cryptography and Network Security, Principles and Practices. Pearson Prentice Hall, 2006.

[92] M. Tarique, K. E. Tepe, S. Adibi, and S. Erfani. Survey of multipath routing protocols for mobile ad hoc networks. Journal of Network and Computer Applications, 32(6): 1125 - 1143, 2009.

[93] L. Viennot and P. Jacquet. Bi-connectivity, $k$-Connectivity and multipoint relays. Research Report RR-6169, INRIA, France, 2007.

[94] J.P. Vilela and J. Barros. A feedback reputation mechanism to secure the optimized link state routing protocol. In 3rd IEEE International Conference on Security and Privacy in Communication Networks (SecureComm), pages 294-303, September 17-20, 2007, Nice, France, September 17 - 202007. 
[95] P.E. Villanueva, T. Kunz, and P. Dhakal. Extending network knowledge: making OLSR a quality of service conducive protocol. In International Conference on Wireless Communications and Mobile Computing (IWCMC), pages 103-108, New York, NY, USA, 2006.

[96] L. Villasenor-Gonzalez, Y. Ge, and L. Lamont. HOLSR: A hierarchical proactive routing mechanism for mobile ad hoc networks. IEEE Communications Magazine, 43(7):118-125, July 2005.

[97] M. Voorhaen, E. Van de Velde, and C. Blondia. MORHE: A transparent multi-level routing scheme for ad hoc networks. In Challenges in Ad Hoc Networking, volume 197 of International Federation for Information Processing (IFIP), K. A. Agha and I. L. Guérin Lassous and G. Pujolle, pages 139-148. Springer Boston, 2006.

[98] P.-J. Wan, K.M. Alzoubi, and O. Frieder. Distributed construction of connected dominating set in wireless ad hoc networks. Mobile Networks and Applications, 9:141-149, 2004. 10.1023/B:MONE.0000013625.87793.13.

[99] B. Wu, J. Chen, J. Wu, and M. Cardei. A survey of attacks and countermeasures in mobile ad hoc networks. In Wireless Network Security, Signals and Communication Technology, Y. Xiao and X. S. Shen and D. Du, editors, pages 103-135. Springer US, 2007.

[100] J. Wu. An enhanced approach to determine a small forward node set based on multipoint relays. In IEEE Vehicular Technology Conference (VTC-Fall), pages 2774-2777, October 2003.

[101] J. Wu, W. Lou, and F. Dai. Extended multipoint relays to determine connected dominating sets in manets. IEEE Transactions on Computers, 55(3):334-347, 2006.

[102] J. Yi, A. Adnane, S. David, and B. Parrein. Multipath optimized link state routing for mobile ad hoc networks. Ad Hoc Networks, 9(1):28 - 47, 2011.

[103] J. Yi, E. Cizeron, S. Hamma, and B. Parrein. Simulation and performance analysis of MP-OLSR for mobile ad hoc networks. In IEEE Wireless Communications and Networking Conference (IEEE WCNC), Las Vegas, US, March 31 - April 3, 2008.

[104] J. Yi, E. Cizeron, S. Hamma, B. Parrein, and P. Lesage. Implementation of multipath and multiple description coding in OLSR. Computing Research Repository (CoRR), abs/0902.4781, 2009.

[105] J. Yi, S. David, H. Adnane, B. Parrein, and X. Lecourtier. Multipath OLSR: simulation and testbed. In 5th OLSR Interop/Workshop, Vienna, Austria, October 2009.

[106] X. Zhou, Y. Lu, and B. Xi. A novel routing protocol for ad hoc sensor networks using multiple disjoint paths. In 2nd Intermational Conference on Broadband Networks (BroadNets), volume 2, pages 944-948, Boston, MA, US, October 2005. 


\section{Index}

$k$-Covered-MPR, 53

UM-OLSR, 17

$k$-Robust-MPR, 57

Algorithms

HCCD, 90

HCCD2, 93

Connected Dominating Set

CDS, 101

DM-OLSR, 101

KCCDS, 101

KRCDS, 101

MP-OLSR, 101

MPRCDS, 101

Control Traffic Messages

CID, 69

Hello, 18

HNA, 21

HTC, 66

MID, 21

TC, 18

MPR, 47, 66

OLSR Implementations

CRC OLSR, 17

NLROLSRd, 17

NOA-OLSR, 17

OLSR, 17

OLSR 11 win, 17

OLSRd, 17

OOLSR, 17

Routing Protocols

AODV, 7

C-OLSR, 67

DSDV, 8

DSR, 7

FSR, 8

HOLSR, 66

LMR, 6

LP-OLSR, 130

MORHE, 67

OLSR, 9

TBRPF, 9

TORA, 6

WRP, 9

ZHLS, 10

ZRP, 10

TC_Redundancy, 50, 81 